\title{
A Recreational Boating Characterization of Brevard County
}

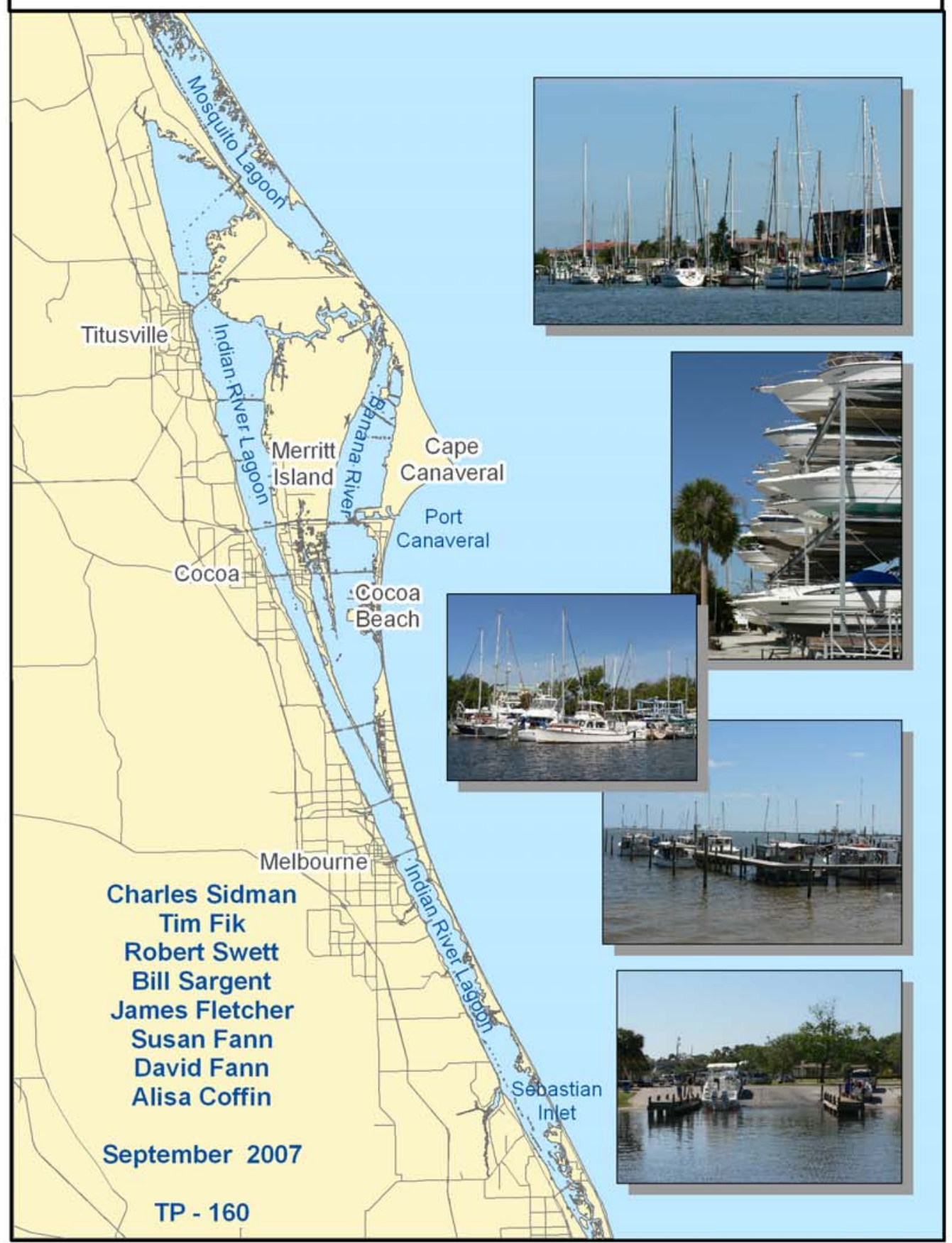



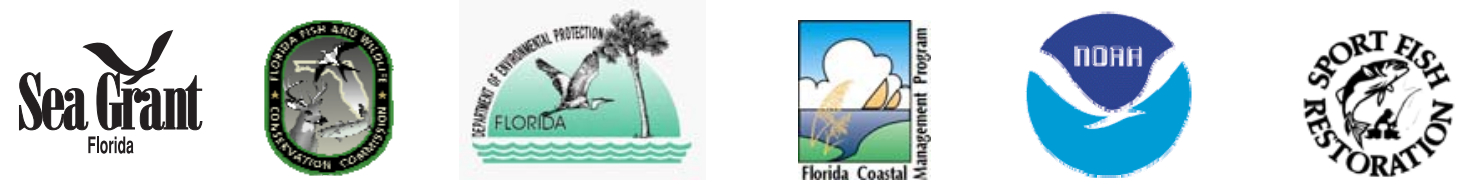

\section{UF| $\mid$ FLINERIIIDA IFAS}

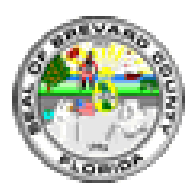

A publication of the University of Florida Sea Grant Program (project number 00059154). The project was funded by Brevard County through the University of Florida Brevard County Extension program, the Florida Fish and Wildlife Conservation Commission Boating and Waterways Section, the Florida Fish and Wildlife Conservation Commission Fish and Wildlife Research Institute, the Florida Department of Environmental Protection, and the Florida Coastal Management Program, pursuant to National Oceanic and Atmospheric Administration award number NA04NOS41900129 with additional support from the Federal Aid in Sport Fish Restoration Program. The views expressed herein are those of the authors and do not necessarily reflect the views of the State of Florida, NOAA or any of their sub agencies. 


\title{
A Recreational Boating Characterization for Brevard County
}

\author{
by \\ Charles Sidman \\ Coastal Planning Specialist \\ Florida Sea Grant \\ University of Florida \\ Tim Fik \\ Associate Professor \\ Department of Geography \\ University of Florida \\ Robert Swett \\ Assistant Professor \\ Department of Fisheries and Aquatic Sciences \\ University of Florida
}

Bill Sargent

Research Scientist

Florida Fish and Wildlife Conservation Commission

Fish and Wildlife Research Institute

St. Petersburg, Florida

James Fletcher

Director, Brevard County Extension

Institute of Food and Agricultural Sciences

University of Florida

Susan Fann

GIS Specialist

Florida Sea Grant

University of Florida

David Fann

Senior GIS Specialist

Florida Sea Grant

University of Florida

Alisa Coffin

Doctoral Candidate

Department of Geography

University of Florida 


\section{Table of Contents}

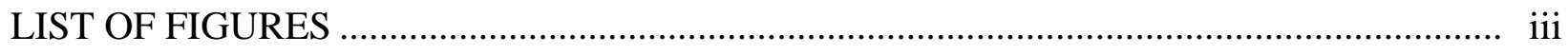

LIST OF TABLES ..............................................................................................

ACKNOWLEDGMENTS ........................................................................................ vii

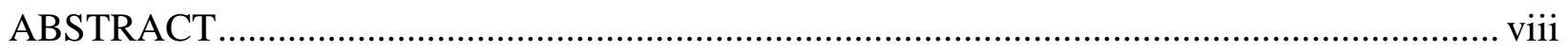

PART 1 - STUDY DESIGN

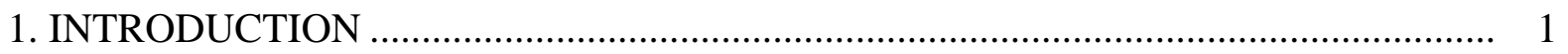

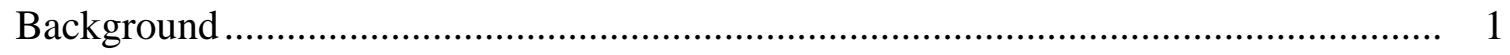

Study Goals and Objectives ..................................................................................... 2

Study Region.................................................................................................................. 3

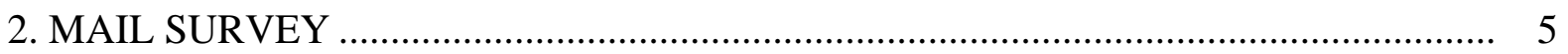

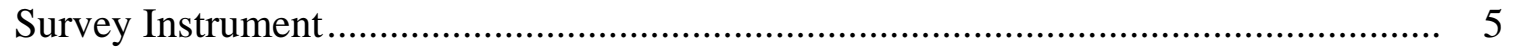

Sample Design .................................................................................................... 6

Identifying Marina, Ramp and Dock Patrons ................................................................. 7

Survey Return Breakdown ............................................................................................ 14

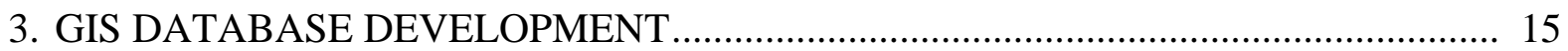

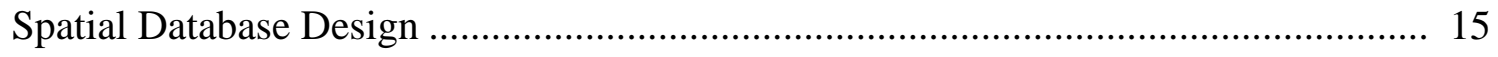

\section{PART 2 - SUMMARY STATISTICS}

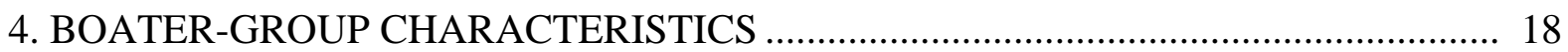

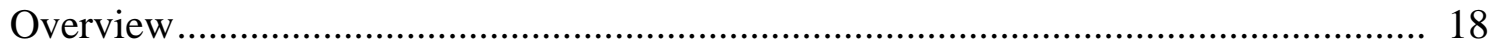

Vessel and Boater Profile: Access Type, Vessel Type, and Boating Experience.......... 18

Rationale for Selecting Departure Sites, and Travel Routes........................................... 33

Boater Activity Profile.............................................................................................. 35

Perceived Congestion................................................................................................... 40

5. SEASONAL BOATING CHARACTERISTICS …...................................................... 42

Defining Boating Seasons - A Temporal Analysis ....................................................... 42

Sample Size Considerations......................................................................................... 45

Validation of Defined Boating Seasons ................................................................... 46

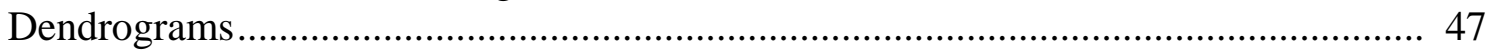

Seasonal Survey Participation....................................................................................... 52

Seasonal Analysis of Number of Trips Taken .............................................................. 53

Seasonal Analysis of Trip Departure-Times............................................................ 60

Seasonal Analysis of Trip Duration......................................................................... 68

Seasonal Analysis of Weekend vs. Weekday Trips....................................................... 78 
Seasonal Analysis of Boating Activities.................................................................... 80

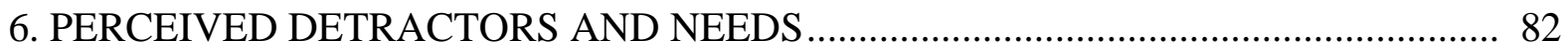

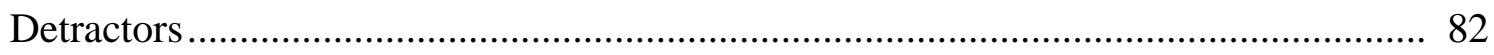

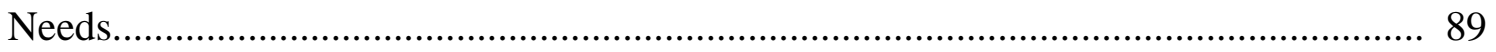

PART 3 - SPATIAL ANALYSIS OF BOATING PATTERNS

7. MAPPING MARINA AND RAMP PATRONAGE …………………………................ 95

Marina and Ramp Use Landside Profile..................................................................... 95

Ramp Use vs. Parking Capacity............................................................................... 98

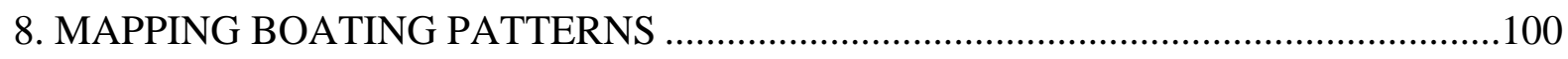

General Clustering Patterns ........................................................................................100

Seasonal Boating Patterns ..........................................................................................104

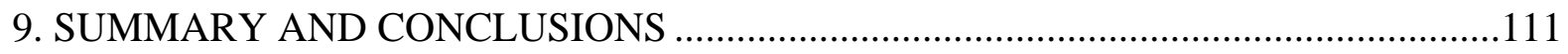

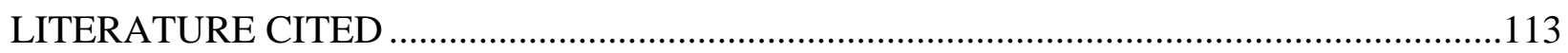

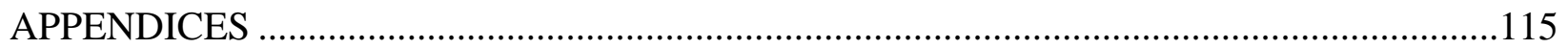

\section{LIST OF FIGURES}

\section{PART 1}

Figure 1. Brevard County Study Area................................................................................ 4

Figure 2. Brevard County Marinas Visited ......................................................................... 9

Figure 3. Brevard County Public Boat Ramps Surveyed........................................................ 11

Figure 4. Spatial Distribution of the Brevard County Dock Sample........................................ 13

Figure 5. Example of GIS Attribute Query and Display: Nature Viewing Spots .................. 16

Figure 6. Example of GIS Attribute Query and Display: Reported Travel Routes................ 17

\section{PART 2}

Figure 1. Histogram and Box Plot of Years Boating Experience (in years). ......................... 22

Figure 2. Histogram and Box Plot Showing the Age of Survey Participants. ....................... 26

Figure 3. Histogram and Box Plot of Drive Times for Boaters Traveling

Three Hours or Less to their Departure Sites ....................................................... 28

Figure 4. Percentage of Survey Respondents Reporting "Yes" to Perceived Congestion By User Group............................................................................................ 41

Figure 5. Mean Monthly Trip Counts (All Survey Respondents)......................................... 44 
Figure 6a. Dendrogram Showing Clusters of Months Based on the Mean Number of Reported Monthly Trips and Related Variables; Using a Multivariate Hierarchical Cluster Analysis

Figure 6b. Dendrogram Showing Clusters of Months Based on the Mean Number of Reported Monthly Trips and Related Variables; Using a Multivariate Hierarchical Cluster Analysis....

Figure 7a. Mean Trip Counts per Month by Season ...................................................... 56

Figure 7b. Monthly Trip-Count Distributions by Season................................................. 56

Figure 8. Mean Trip Counts by Season and User Group. ............................................. 59

Figure 9. Median Trip Counts by Season and User Group. ............................................ 59

Figure 10a. Histogram of AM Departure Time-Non-Peak Season................................... 62

Figure 10b. Histogram of AM Departure Time-Peak Season .......................................... 62

Figure 10c. Histogram of AM Departure Time-Shoulder Season. .................................... 63

Figure 11. Mean AM Departure Times by User Group and Season ................................... 64

Figure 12. Frequency Distribution of PM Launch Times for Reported Trips ...................... 66

Figure 13a. Mean Reported Duration of Day Trips (in hours) by Season/User Group ........... 70

Figure 13b. Box-Plot of the Duration of “Day Trips” as Reported by respondents from all user groups ......................................................... 71

Figure 14a. Mean Reported Duration of Overnight Trips by Season and User Group

(Trip duration $>24$ hours).

Figure 14b. Mean Reported Duration of Overnight Trips by Season and User Group

(Trip duration $>24$ hours and $\leq 168$ hours). 76

Figure 14c. Box Plot Showing Duration of Reported Overnight Trips by Survey

Respondents from All User Groups (Duration $>24$ hours and $\leq 168$ hours).... 77

\section{PART 3}

Figure 1. Florida Distribution of Brevard County Ramp and Marina Patrons..................... 96

Figure 2. Florida Distribution of Brevard County Marina Patrons .................................. 97

Figure 3. Florida Distribution of Brevard County Ramp Patrons ..................................... 98

Figure 4. Core Service Areas for the Freddie Patrick Park Boat Ramp .............................. 99

Figure 5. Travel Corridors as Summarized with the GIS ...............................................102

Figure 6. Favorite Destinations as Summarized with the GIS ........................................103 
Figure 7. Congested Areas as Summarized with the GIS ......................................................104

Figure 8. Close-up of Favorite Destination Spots for the Merritt Island Area........................105

Figure 9. Destination Clustering for the "Peak" Boating Season.............................................108

Figure 10. Destination Clustering for the "Off-Peak" Season .....................................................109

Figure 11. Destination Clustering for the "Shoulder" Season.....................................................110

\section{LIST OF TABLES}

\section{PART 1}

Table 1. Breakdown of Vessel Bow Numbers Collected from Marinas................................ 8

Table 2. Breakdown of Ramp Survey Dates and Tag Numbers Collected............................ 10

Table 3. Survey Return Breakdown................................................................................. 14

Table 4. Trip Features Digitized from Returned Surveys ..................................................... 15

\section{PART 2}

Table 1. Survey Response by Waterway Access Category .................................................. 19

Table 2. Vessel Used for Reported Trips............................................................................ 19

Table 3. Mean Length and Draft by Departure/Water Access Category ............................... 20

Table 4. Mean Number of Months Resident in Florida by Water Access Category ............ 21

Table 5. Years Boating Experience in Florida (all survey respondents) ............................... 22

Table 6. Years of Boating Experience in Florida by Waterway Access Category ............... 23

Table 7. Boaters Having Completed a Boat Safety/Seamanship Course by Waterway Access Category .......................................................................... 24

Table 8. Age of Boaters by Waterway Access Category ....................................................... 25

Table 9. Drive Time from Home to Departure Site for Reported Trips (in minutes)........... 28

Table 10. Mean Departure Time by Waterway Access Category .............................................. 29

Table 11. Trip Duration: On-Water Travel Time by Waterway Access Category (All Reported Day Trips; Trip duration $\leq 24$ hours) ............................................... 30

Table12. Trip Duration: On-Water Travel Time by Waterway Access Category (All Reported Overnight Trips; Trip duration $>24$ hours and < 168 hours) ......... 31

Table 13. Weekday vs. Weekend Trips by Water Access Category ....................................... 32

Table 14. Reasons for Selecting a Favorite Departure/Launch Site ......................................... 33

Table 15. Reasons for Selecting a Favorite Travel Route........................................................ 34 
Table 16. Boaters’ Activity Statistics (entire sample/all respondents) ................................ 35

Table 16a. Boaters’ Activity Statistics: Boat Ramp Group ............................................. 36

Table 16b. Boaters’ Activity Statistics: Marina Wet Slip Group ...................................... 37

Table 16c. Boaters’ Activity Statistics: Marina Dry Storage Group .................................. 38

Table 16d. Boaters’ Activity Statistics: Dock Group ....................................................... 39

Table 17. Proportion of Boaters that Indicated They Had Avoided or Left Their Favorite Spots/Destinations Due to Congestion................ 41

Table 18. Mean Number of Reported Trips by Month and User Category ......................... 43

Table 19. A Listing and Description of Variables Used in the Hierarchical Cluster Analysis ............................................................. 46

Table 20. Results of Cluster Analysis in the Designation of Boating Seasons.................... 48

Table 21. Seasonal Survey Participation among User Groups (Reported Trips) ................. 52

Table 22. Seasonal Breakdown of the Mean and Median Number of Trips per Month by Waterway Access/User Group..................................................................... 54

Table 23. Kruskal-Wallis ANOVA and Mean Rank Sum Test Results ............................. 57

Table 24. Mean AM Departure Time by Season and User Group..................................... 61

Table 25. Mean PM Departure Time by Season and User Group ....................................... 67

Table 26. Mean and Median Trip Durations (in hours) by Season and User Group--Reported “Day Trips” (Trips $\leq 24$ Hours)....................................69 69

Table 27. Mean and Median Overnight Trip Durations (in hours) by Season and User Group--“Overnighters” (Trips > 24 Hours and $\leq 168$ Hours)................. 75

Table 28. Proportion of "Weekend Trips” by User Group and Season .............................. 79

Table 29. A List of Boating Activities (as provided in the Survey) .................................. 81

Table 30. Percentage of Reported Boating Activities by Season (\% based on Typical Trip/Month/Season—weighted by number of trips)............. 81

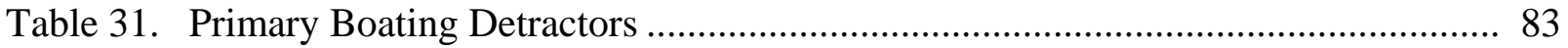

Table 32. Primary Detractors by Waterway Access Group ............................................ 88

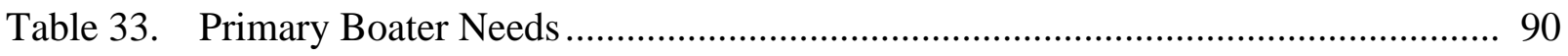

Table 34. Primary Needs by Waterway Access Group................................................... 94

\section{PART 3}

Table 1. Ramp Parking Capacity and Utilization Estimates. 100 


\section{Acknowledgments}

The Brevard County Extension Service, The Florida Fish and Wildlife Conservation Commission Fish and Wildlife Research Institute (FWC FWRI), and the FWC Boating and Waterways Section funded this project through coordinated grants from Brevard County and the Florida Department of Environmental Protection Florida Coastal Management Program (FDEP FCMP), pursuant to National Oceanic and Atmospheric Administration (NOAA) award number NA06NOS41900129.

The success of this project relied upon the individual contributions of various team members. Principal Investigators Charles Sidman and Robert Swett (Florida Sea Grant) were responsible for project administration, overall design of the questionnaire and correspondence, sample selection, the GIS database design, and reporting. Timothy Fik (Department of Geography, University of Florida) performed the statistical analyses. Susan Fann (Florida Sea Grant) conducted the content analysis of the primary detractors and needs questions. Alisa Coffin conducted the spatial analysis of seasonal use. Bill Sargent (FWRI) served as the Florida Wildlife Conservation Commission project liaison and contributed to the questionnaire, survey design, and reporting. David Fann was responsible for the questionnaire map layout and design, and assisted with the cartography. Lachlan Smith conducted the marina survey. Lachlan Smith, Lou Ann Wendt and County Parks and Recreation Staff conducted the ramp surveys. Eddie Leonard (Brevard County Marine Agent), Liz Alward (formerly with Brevard County Parks and Recreation) coordinated the ramp surveys with Brevard County Parks and Recreation Staff. Liz McDuffee (assistant to the Brevard County Natural Resource Director) provided a valuable editorial review of the interim and final reports. Dick Tudor and James Harrison of Smart Mail Inc. (Alachua, Florida) implemented the mailings. Susan Fann, Rick Abella, Daniel Godwin, and Corina Guevara managed and processed attribute and spatial data from the returned questionnaires. Special recognition is due to James Fletcher (Brevard County Extension Director) and to Ernie Brown (Brevard County Natural Resource Director) who acted as local coordinators for the project.

We especially thank the Brevard County Parks and Recreation staff for conducting the year-long boat ramp surveys and the many boaters who donated their time to complete and return the questionnaires. It is our intention that this work will advance the County's Comprehensive Maritime Management Master Plan effort and provide information products and outreach efforts of benefit to boaters who use Brevard County waterways. 


\section{Abstract}

Brevard County faces the difficult yet critical management challenge of how to sustain economic viability while maintaining the integrity of its coastal environmental resources. Recreational boating and waterway access figure prominently within this multi-faceted challenge. The County recognizes that effective coastal community planning requires the availability of pertinent and accurate information concerning on-water activities, using best technology and scientific methods. A recreational boating use study was, therefore, recommended as a means to document and map present marine facility and waterway usage in support of Brevard County's Comprehensive Maritime Management Master Plan effort. The recreational boating study described by this report resulted from a collaborative partnership between the Florida Fish and Wildlife Conservation Commission (FWC), the Brevard County Department of Natural Resources, the University of Florida Brevard County Cooperative Extension Program, and the University of Florida Sea Grant College Program.

This report documents the methods, procedures, and results of a map-based mail survey that was distributed in three waves (June/July 2006, November 2006, and March 2007) to 11,916 Brevard County boaters (some participating boaters received up to three questionnaires over the year-long study period) to obtain seasonal information about their boating preferences, use profiles, and travel patterns. Boaters were categorized according to the type of facility that they used to access the waterway: marina wet slip, marina dry storage, public ramp, and private dock.

Questionnaire recipients were asked to mark the start and end point of their last two recreational boating trips, draw their travel routes, and identify their favorite boating destinations and primary activities that they engaged in at destinations. Data collected from 3,367 returned surveys over the three survey waves (2,480 of which were associated with unique users) were digitized into the ESRI ArcGIS geographic information system (GIS). This translated to a sample of 6,307 travel routes, 6,307 trip departure sites (origins), 7,437 favorite boating destinations, and 2,907 locations of perceived congestion, and represented an overall return rate of $19 \%$.

This study differs from previous efforts to characterize (i.e., profile and describe) boating patterns in its spatial approach. Thousands of individual boating trips were described and drawn by boaters on maps. The spatial information was then entered into a GIS. The descriptive data about boaters' trips —-, including preferences for selecting trip departure sites, destinations, and travel routes, favorite activities, vessel types, and the timing, duration, and frequency of tripscan be linked to the data within the GIS, for further analysis.

Information products generated from this study include:

1. A profile of boaters who use Brevard County waterways for recreation;

2. A profile of the types of recreational vessels operated on Brevard County waterways; 
3. A description and location of the types of recreational activities that take place on Brevard County waterways;

4. A description of boater preferences for waterway access facility amenities (e.g., marinas and ramps);

5. A summary of principal detractors and needs related to Brevard County waterways;

6. Spatial data formatted within a GIS that can be used to map;

a. service areas for Brevard County boating facilities

b. departure or launch sites

c. favorite water-based boating destinations and the activities that occur there

d. trip routes that define where Brevard County boaters travel on the water

e. areas of perceived waterway congestion

7. An evaluation of seasonal aspects for many of the information products listed above.

This report is divided into three parts. Part 1 discusses the survey design, mailing implementation, and data collection. Part 2 presents the results of a statistical analysis of survey questions and compares seasonal differences in use among users of marinas (wet slips and dry storage facilities), ramps, and private docks. In addition, a content analysis of the two openended questions that asked about "detractors" and "needs" identifies important issues from the perspective of the Brevard County boating community. Part 3 presents the results of spatial analyses of land-side and water-side use patterns. First, ramp and marina facility patronage was evaluated and mapped. Second, a GIS density function identified travel corridors, favorite destination locales and areas of perceived congestion. Last, the Getis \& Ord G-Statistic was employed to identify and map seasonal use patterns of boating destination "hot-spots" indicated by survey respondents. 


\section{Part 1- Study Design}

\section{Chapter 1. Introduction}

\section{Background}

Boating is a key element in Florida's coastal lifestyle and growth phenomena. Florida currently ranks first in the nation in recreational boat registrations, with 946,072 pleasure boats registered or titled, according to the National Marine Manufacturers Association (2005). This represents approximately one boat for every 17 residents. Of equal note, Florida is the number one U.S. destination for marine recreation-including saltwater boating-with an estimated 4.3 million participants (Leeworthy \& Wiley, 2001). Coastal development, the ever-increasing number of boaters, and the diversity of recreational boating activities that now take place within Florida's coastal bays, estuaries, and waterways have had positive economic impacts, but also have profoundly altered the coastal estuarine environment (Letson, 2002; Antonini, Fann \& Roat, 1999). As demand for the use of Florida's waterways increases, so does the need for enhanced public access, maintenance of waterway infrastructure, public safety, and environmental protection. There is, however, little information available to resource managers and planners that describes the preferences and use patterns of the boating community.

Brevard County is a microcosm of Florida’s growth phenomena. The County faces the difficult yet critical management challenge of how to sustain economic viability while maintaining the integrity of coastal environmental resources. Recreational boating and waterway access figure prominently within this multi-faceted challenge. With the county's steady population growth (53\% increase since 1985$)^{1}$ has come a concurrent increase in recreational boat registrations (73\% increase since 1985) ${ }^{2}$ and the associated demands placed on coastal and marine resources. Further, the diverse and often competing uses of coastal resources have increased the number and intensity of user conflicts. For instance, privatization and conservation forces contend with recreational boating for waterway access. Given that recreational boating is a major contributor to Florida's economy (an estimated $\$ 18$ billion annually ${ }^{3}$ ), a decline in access becomes a particularly pressing issue in the management challenge.

For sustained application, community development and resource management strategies must be compatible and equitable. As demand for access to and use of Brevard County waterways increases, so then does the need for a better understanding and detailing of present usage and its impacts. Brevard County realizes that effective coastal community planning requires the availability of pertinent and accurate information concerning on-water activities, using best technology and scientific methods.

\footnotetext{
${ }^{1}$ According to the Bureau of Economic and Business Research (Florida Statistical Abstract 2006), the Brevard County population grew from 339,473 in 1985 to 521,422 in 2005, which represents an increase of 181,949 people.

${ }^{2}$ According to the Bureau of Economic and Business Research (Florida Statistical Abstract 2006), recreational vessel registrations in Brevard County grew from 19,745 in 1985 to 34,234 in 2005; an increase of 14,489 pleasure craft.

${ }^{3}$ Murray, T.J. Virginia Institute of Marine Science, Gloucester Point, Virginia. Personal communication on behalf of the Marine Industries of Florida (www.boatflorida.org).
} 
Science-based data pertaining to recreational boating patterns should include spatially explicit referenced details. For example, boating origin analyses, with respect to the type of access facility, facility location, and user numbers, are necessary for informed policy decisions as to infrastructure siting (e.g., public ramps). The knowledge of boater activities and destinations facilitates planning with respect to both impact considerations and optimal waterway use (e.g., dedicated water-sport areas). Finally, spatial analyses of boat traffic from origin through destination locales figure in such determinations as waterway service levels (e.g., dredging), and appropriate regulatory input. A scientific approach provides information for rational and objective planning to assure that future economic viability and environmental protection needs are balanced.

\section{Study Goals and Objectives}

The goals of the study were (1) to quantify and map public access facility use through an inventory of marinas and boat ramps, and (2) to obtain information from boaters who use Brevard County access facilities and waterways regarding their preferences, activities, and water-use patterns. Specific objectives included (1) the development of spatial data sets within a geographic information system (GIS) to map boating patterns, and (2) the analysis of trip information provided by boaters to describe the preferences and behaviors of boaters who use Brevard County waterways. Examples of the information products derived from the study are as follows:

1. A profile of boaters who use Brevard County waterways for recreation, and characteristics of their trips (e.g. timing, frequency, and duration);

2. A profile of the types of recreational vessels operated on Brevard County waterways;

3. A description and location of the types of recreational activities that take place on Brevard County waterways;

4. A determination of preferences for waterway access facility amenities (e.g., ramps);

5. A summary of principal detractors and needs related to Brevard County waterways;

6. A compilation of spatial boating trip data within a GIS that can be used to map;
a. service areas for Brevard County boating facilities
b. departure or launch sites
c. favorite water-based boating destinations and the activities that occur there
d. trip routes that define where Brevard County boaters travel on the water
e. areas of perceived waterway congestion

7. An evaluation of seasonal aspects for many of the information products listed above.

Information obtained from this recreational boating pattern analysis will figure in the development of the County's Comprehensive Maritime Management Master Plan, and can serve to advance objectives pertaining to a variety of waterway management issues. Examples of ways 
that boating pattern information can be used to improve public waterway access and aquatic resource management and to address boaters' concerns include the following:

1. Categorization and spatial representation of boater departure sites, routes, and destinations to address community concerns regarding waterway access, maintenance, signage, and facility siting;

2. Comparison of boating information with other spatial (GIS) data layers (e.g., environmental, development patterns) to help guide resource and public safety management;

3. Identification of temporal and activity-derived spatial profiles to map boating pressure "hot-spots" on county waterways;

4. Identification of boating-related problems and their solutions as input to management strategies, education programs, and communications products that target available resources to issues of greatest concern;

5. Determination of service areas for public launching facilities (e.g., boat ramps) and the demand placed on those facilities from county residents and visitors.

The study process involved (1) the development of a survey instrument and accompanying correspondence; (2) the identification of target boater groups by waterway access facility type; (3) the implementation of seasonal mail surveys of target boater groups; (4) the construction of spatial databases from returned mail surveys that identify trip departure sites, destinations, travel routes, and congested areas, and (5) the determination of seasonal boating profiles. The process was consistent with previous boating pattern studies conducted by Florida Sea Grant and the Florida Fish and Wildlife Conservation Commission's Fish and Wildlife Research Institute for Tampa and Sarasota Bays (Sidman \& Flamm, 2001; Sidman, Fik \& Sargent, 2004); the Greater Charlotte Harbor (Sidman, Swett, Fik, S. Fann, D. Fann \& Sargent, 2005); and Sarasota County (Sidman, Swett, Fik, Fann \& Sargent, 2006). A similar recreational boating pattern study for Bay County, Florida is currently underway.

\section{Study Region}

The Brevard County study region extends approximately 70 miles from Oak Hill (Mosquito Lagoon) in Volusia County in the north to the Wabasso Island area of Indian River County to the south. An estimated 34,234 pleasure boats are currently registered in the county ${ }^{4}$. This does not include the many thousands of boaters who travel to the area from other Florida counties. Recreational boaters are attracted to this region by its many mangrove islands and protected waters that provide excellent opportunities for small-craft fishing, nature viewing, and picnicking/socializing along spoil island beaches and exposed sand spits. The study region comprises roughly 2,800 square miles of the Atlantic Ocean, and interior bay waters that include the Mosquito Lagoon, the Indian River Lagoon, and the Banana River (Figure 1).

\footnotetext{
${ }^{4}$ Bureau of Economic and Business Research (Florida Statistical Abstract 2006).
} 


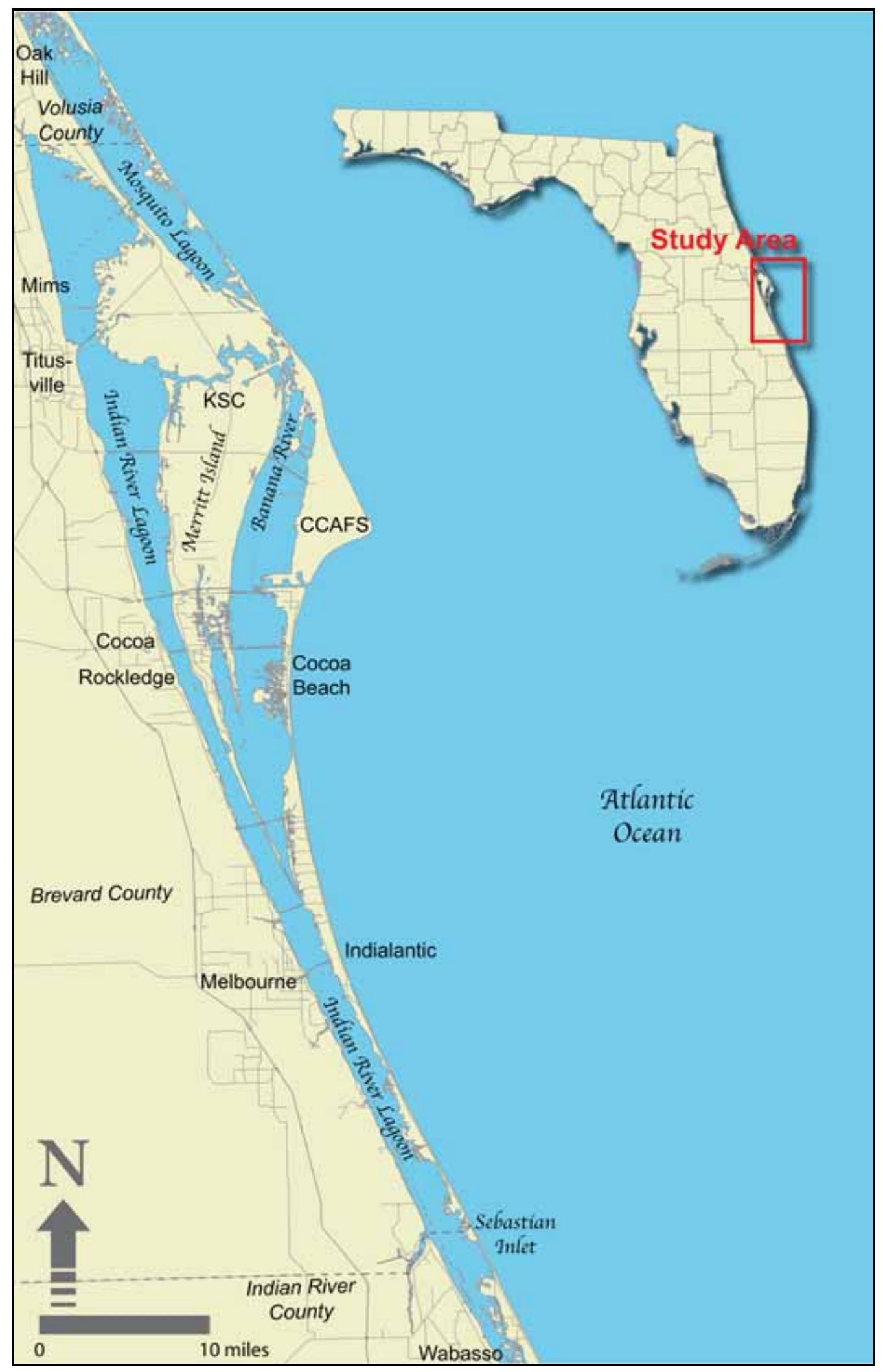

Figure 1. Brevard County Study Area. 


\section{Chapter 2. Mail Survey}

\section{Survey Instrument}

The survey questionnaire developed for this study was patterned after similar, previous studies (Falk, Graefe, Drogin, Confer \& Chandler, 1992; Sidman \& Flamm, 2001; Sidman, et al. 2004; West, 1982;) and was designed to (1) capture spatial information regarding trip departure sites, favorite boating destinations, intervening travel routes, and congested areas; (2) characterize boaters with respect to the types of vessels owned and used, activity preferences, and the timing, frequency and duration of their recreational outings; and (3) identify problems and needs from the perspective of the boating community (see Appendix A for the survey instrument and associated correspondence).

The survey instrument was a two-sided $22 \mathrm{X} 34$ inch questionnaire that folded to $8.5 \mathrm{X} 11$ inches. The questionnaire contained a map (1:160,000 scale; 1 inch approximating 2.5 miles) of the Brevard County boating region on one side; the reverse side consisted of 25 questions divided into the following topical areas:

1. Description of last two pleasure boating trips

2. Description of typical boating trips

3. Description of survey respondent

The following additional items were included with each mailed questionnaire.

1. A cover letter that explained the study

2. A Brevard County Boater's Guide developed by the FWC FWRI

3. A postage paid return envelope with postal permit indicium

4. A mailing envelope that included return address and postage permit indicium

In addition, a 4 X 6 card was mailed approximately two weeks after each mailing as a reminder to survey recipients to complete and return the questionnaire.

A beta-version of the survey instrument was provided to Brevard County Natural Resources staff and mailed to six local boating experts who agreed to review and complete the questionnaire. Reviewer comments and suggestions were used to improve the content of the questionnaire.

The questionnaire asked survey recipients to mark, on the map-side, the location of the departure sites, travel routes, and favorite destinations associated with their last two pleasure boating trips. In addition, survey recipients identified locations where they experienced congestion, defined as "more boaters than they preferred." Complementary questions on the text side of the survey allowed recipients to characterize their last two trips according to vessel type, the departure weekday, month, and time, and the time spent on the water. In addition, recipients were asked to characterize and name the departure sites of their last two trips and to rank reasons for departure site selection where this differed from a home dock. With respect to typical trips, respondents were asked to give the number of days per month that they had taken trips during the 
past year and the typical activities they had pursued. They were also asked to identify and rank reasons for selecting travel routes. Finally, a series of questions sought to characterize the respondent in terms of age and boating experience. This included two open-ended questions asking for principal detractors and needs in their Brevard boating experience.

\section{Sample Design}

The sample design was developed to acquire group-specific information that could be used to compare and contrast use patterns among the four discrete Brevard boater groups associated with the following access facilities: (1) marina wet slips, (2) dry storage facilities, (3) public boat ramps, and (4) private docks.

It was determined that a minimum sample size of 384 was required to statistically represent each of the four boater groups. This sample size was considered adequate, based on a tolerable error of \pm 0.05 and a confidence level of 95 percent, for a population that is finite and does not exceed 2,000,000 (McCall, 1982). A gross sample of 2,000 boaters for each of the four groups was therefore targeted to obtain 384 returns from each group. This ratio assumes a return rate of approximately 20 percent, based on return rates from previous surveys of southwest Florida boaters (Antonini, Zobler, Sheftall, Stevely \& Sidman, 1994, Antonini, West, Sidman \& Swett, 2000; Sidman \& Flamm, 2001; Sidman, et al. 2004; Sidman, et al. 2006).

Questionnaires were distributed to area boaters in three waves. The first was mailed during June - July of 2006; the second in November 2006; the third in March 2007. Due to the small number of participating marinas and dry storage facilities in Brevard County, the minimum sample of 2,000 users each was not achieved. Shortfalls for the marina wet and dry storage groups, however, enabled more surveys to be assigned to ramp users, a much larger identified user group. An adequate sample, exceeding the 2,000 minimum, was identified for the dock access group.

The second wave (November 2006) consisted of two survey types, one the original and the other an abridged version (not the full complement of questions). The latter went to those boaters who had completed the first survey and had indicated a willingness to participate in future surveys $^{5}$. The original survey was re-sent to all dock and marina users who had not returned the first (nor indicated an unwillingness or inability to participate), in an effort to capture additional input from relatively fixed user populations. In addition, ramp users newly observed during those months comprising the second seasonal observation period received a questionnaire with the full complement of questions.

The third wave of surveys (March 2007) also consisted of two survey types. Again, the first consisted of an abridged survey to those boaters who responded to either the first or second wave of surveys with a willingness to participate in future surveys (note that only a portion of previously participating ramp users were mailed the abridged version, given the new ramp user sample identified by ongoing visits to boat ramps). The original survey format (full complement

\footnotetext{
${ }^{5}$ It was determined that an abridged (i.e., shortened) version of the survey, mailed to respondents who had already completed a longer version of the questionnaire, would adequately capture seasonal trip information and reduce redundancy related to answering the same questions multiple times.
} 
of questions) went out to all marina and dock users who had not responded to either the first or second mailings, together with those ramp users newly identified during the winter months.

\section{Identifying Marina, Ramp, and Dock Patrons}

Vessel, automobile, and boat trailer registration numbers collected at area marinas and boat ramps were used to obtain names and mailing addresses from the State's Vehicle Title Registration System (VTRS), maintained by the Florida Department of Highway Safety Motor Vehicles (DHSMV), for the marina wet slip, dry storage facility, and public ramp samples. The names and addresses of owners of documented vessels observed at marinas were obtained from the United States Coast Guard Documented Vessel database that is available on-line. In addition, VTRS records were compared with Brevard County tax rolls to identify owners of waterfront parcels who also owned a boat (i.e., dock owners).

During March - April 2006 Florida Sea Grant personnel visited 34 marinas (Figure 2) located in Brevard County to record bow numbers from vessels stored in wet slips and in dry storage facilities (and outside dry dock areas). Florida Sea Grant personnel were granted access to 28 marinas and logged bow numbers from 883 vessels moored in wet slips and 451 vessels kept in dry storage facilities (Table 1). Bow numbers were compared to the State's VTRS, yielding owner names and mailing addresses for 747 wet slip and 350 dry storage facility users. The name and hailing port of documented vessels were also obtained and used to acquire additional names and addresses from the United States Coast Guard Documented Vessel Database. A total of 1,089 of those addresses were validated as ‘deliverable’ by US Postal Service software. In order to maintain the privacy of their customers six of the marinas requested that they mail the surveys to their clients. A total of 500 survey packets were distributed to those marinas. 
Table 1. Breakdown of Vessel Bow Numbers Collected from Marinas.

\begin{tabular}{|c|c|c|c|c|}
\hline $\begin{array}{l}\text { Brevard County } \\
\text { Marina's Surveyed }\end{array}$ & $\begin{array}{l}\text { Wet Slip } \\
\text { Capacity }\end{array}$ & $\begin{array}{c}\text { Wet Slips } \\
\text { Inventoried }\end{array}$ & $\begin{array}{l}\text { Dry Storage } \\
\text { Capacity }\end{array}$ & $\begin{array}{l}\text { Dry Storage } \\
\text { Inventoried }\end{array}$ \\
\hline Harbor Square Marina & 120 & 83 & 0 & 0 \\
\hline Banana River Marine & 66 & 32 & 65 & 32 \\
\hline Jay's Harbor Lights Marina & 28 & 26 & 8 & 5 \\
\hline Funtime Boats & 0 & 0 & 50 & 47 \\
\hline Cape Marina & 115 & 66 & 160 & 156 \\
\hline Pines Resort & 42 & 28 & 0 & 0 \\
\hline KARS Park & 10 & 2 & 0 & 0 \\
\hline Port Canaveral (Cocoa Beach) Yacht Club & 50 & 27 & 0 & 0 \\
\hline Sebastian Inlet Marina & 0 & 0 & 35 & 22 \\
\hline Waterline Marina & 102 & 62 & 0 & 0 \\
\hline Kennedy Point Yacht Club & 110 & 35 & 40 & 12 \\
\hline Telemar Bay Marina & 200 & 73 & 125 & 32 \\
\hline Orange Cove Marina & 30 & 13 & 0 & 0 \\
\hline Eau Gallie Yacht Basin & 60 & 55 & 0 & 0 \\
\hline Westland Marina & 86 & 34 & 60 & 46 \\
\hline Anchorage Yacht Basin & 85 & 52 & 100 & 61 \\
\hline Pineda Point Marina & 30 & 29 & 72 & *Declined Access \\
\hline Sebastian Inlet State Park Marina & 30 & 15 & 20 & 2 \\
\hline Titusville Municipal Marina & 203 & 147 & 0 & 0 \\
\hline Treasure Coast Marina and Boatyard & 50 & 33 & 0 & 3 \\
\hline Sebastian River Marina \& Boat Yard & 70 & 41 & 65 & 33 \\
\hline Melbourne Yacht Club & 40 & 30 & 0 & 0 \\
\hline TOTALS & 1,527 & 883 & 800 & 451 \\
\hline \multicolumn{5}{|l|}{ Declined to Participate - Not Surveyed } \\
\hline \multicolumn{5}{|l|}{ Pelican Harbor Marina } \\
\hline \multicolumn{5}{|l|}{ Palm Bay Marina } \\
\hline \multicolumn{5}{|l|}{ Eau Gallie Yacht Club } \\
\hline \multicolumn{5}{|l|}{ *Pineda Point Dry-Storage } \\
\hline \multicolumn{5}{|l|}{ Intracoastal Marina } \\
\hline \multicolumn{5}{|l|}{ Scorpion } \\
\hline $\begin{array}{l}\text { Agreed to Distribute Surveys to Clients } \\
\text { - Not Surveyed on Site }\end{array}$ & $\begin{array}{l}\text { \# Surveys } \\
\text { Distributed }\end{array}$ & & & \\
\hline Harbortown Marina & 80 & & & \\
\hline Manatee Cove Marina (PAB) & 165 & & & \\
\hline Melbourne Harbor Marina & 100 & & & \\
\hline San Sebastian Yacht Club and Marina & 50 & & & \\
\hline Treasure Coast Marina & 25 & & & \\
\hline Sunrise & 80 & & & \\
\hline TOTAL & 500 & & & \\
\hline
\end{tabular}




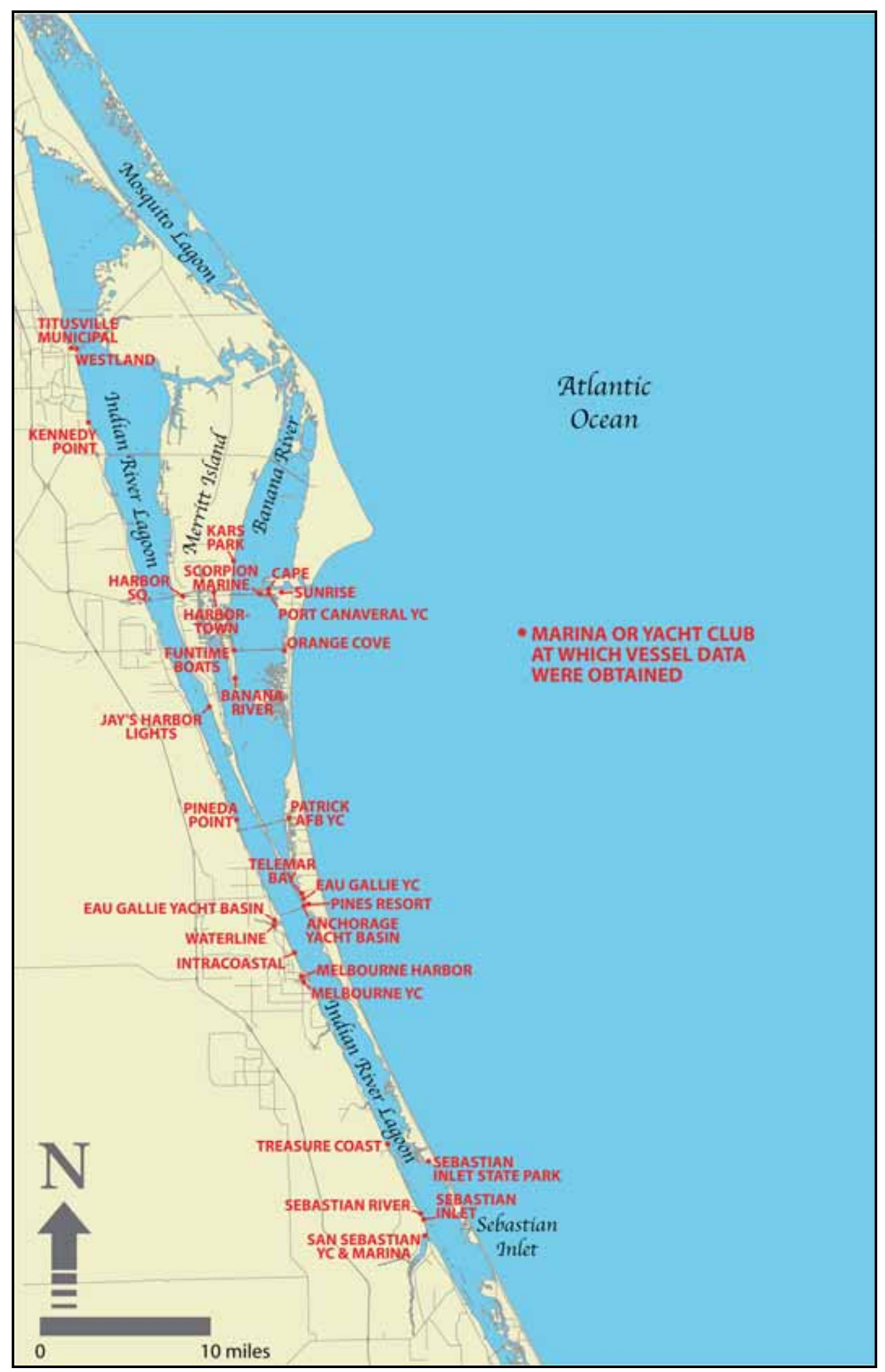

Figure. 2. Brevard County Marinas Visited. 
Brevard County Parks and Recreation staff visited 30 area ramps (Figure 3) to collect license plate numbers associated with boat trailers and corresponding tow vehicles observed in ramp parking areas. The collection period ran for one year (from March 2006 - February 2007), ${ }^{6}$ during which time 22,018 new and repeat users were observed (Table 2). Trailer and tow vehicle information was compared to vessel trailer and vehicle registration databases, to yield corresponding names and mailing addresses for ramp patrons. A total of 4,397 unique (nonrepeat) ramp patrons (of 4,636 unique tag numbers identified at ramps - see Table 3) were sent surveys in June/July 2006. A total of 1,767 unique ramp patrons received a survey during November 2006, and 1,657 received a survey during March of 2007. A total of 8,060 ramp users (of 9,025 unique addresses obtained during the survey period) received a questionnaire.

Table 2. Breakdown of Ramp Survey Dates and Tag Numbers Collected.

\begin{tabular}{|l|c|c|c|}
\hline Ramp Name & \# Dates Surveyed & Avg. Count / Day & \# Tags Observed \\
\hline 1st Street & 51 & 2 & 98 \\
\hline Alex Goode Park & 51 & 5 & 251 \\
\hline Bairs Cove & 49 & 29 & 1440 \\
\hline Ballard Park & 50 & 20 & 993 \\
\hline Bio Lab & 48 & 8 & 374 \\
\hline Constitution Bicentennial Park & 43 & 7 & 303 \\
\hline Eau Gallie Causeway & 50 & 13 & 656 \\
\hline Eddy Creek & 48 & 4 & 181 \\
\hline Freddie Patrick Park & 42 & 55 & 2292 \\
\hline Front Street & 54 & 9 & 502 \\
\hline H Pollak Park & 51 & 6 & 326 \\
\hline John Jorgensen Landing & 51 & 26 & 1303 \\
\hline Kelly Park & 49 & 26 & 1132 \\
\hline Kennedy Point Park & 42 & 17 & 711 \\
\hline Kiwanis Island Park & 44 & 7 & 338 \\
\hline Lee Wenner & 37 & 16 & 574 \\
\hline Long Point Park & 39 & 3 & 109 \\
\hline Main Street Boat Dock & 46 & 16 & 735 \\
\hline Marina Park & 44 & 13 & 568 \\
\hline Mims & 44 & 7 & 289 \\
\hline Old Beacon 42 Camp & 48 & 21 & 1003 \\
\hline Old Scottsmoor & 44 & 8 & 346 \\
\hline Parrish Park & 48 & 39 & 1871 \\
\hline Pineda Landing & 43 & 12 & 520 \\
\hline Port End Park & 43 & 11 & 456 \\
\hline Port St John & 46 & 15 & 711 \\
\hline Ramp Road Park & 41 & 7 & 295 \\
\hline Sebastian Inlet St. Rec. Facility & 37 & 27 & 998 \\
\hline Sebastian Yacht Club & 46 & 39 & 839 \\
\hline Wabasso Causeway Park & 45 & 19 & $\mathbf{2 0 1 8}$ Trailer Tags \\
\hline SUMMARY TOTALS & $\mathbf{5 4}$ Survey Dates & $\mathbf{1 6}$ & $\mathbf{9}$ \\
\hline
\end{tabular}

*Total number of trailers/tow vehicles observed at ramps on all survey dates. The same vessel trailer or tow vehicle could be observed on multiple occasions at the same or different ramp.

\footnotetext{
${ }^{6}$ Ramps were visited on 54 days throughout the one-year survey period (see appendix B for the survey schedule). Unfortunately, not all ramps were surveyed on the pre-determined dates. As a result, there are gaps in the data collected for all but one ramp (Front Street).
} 


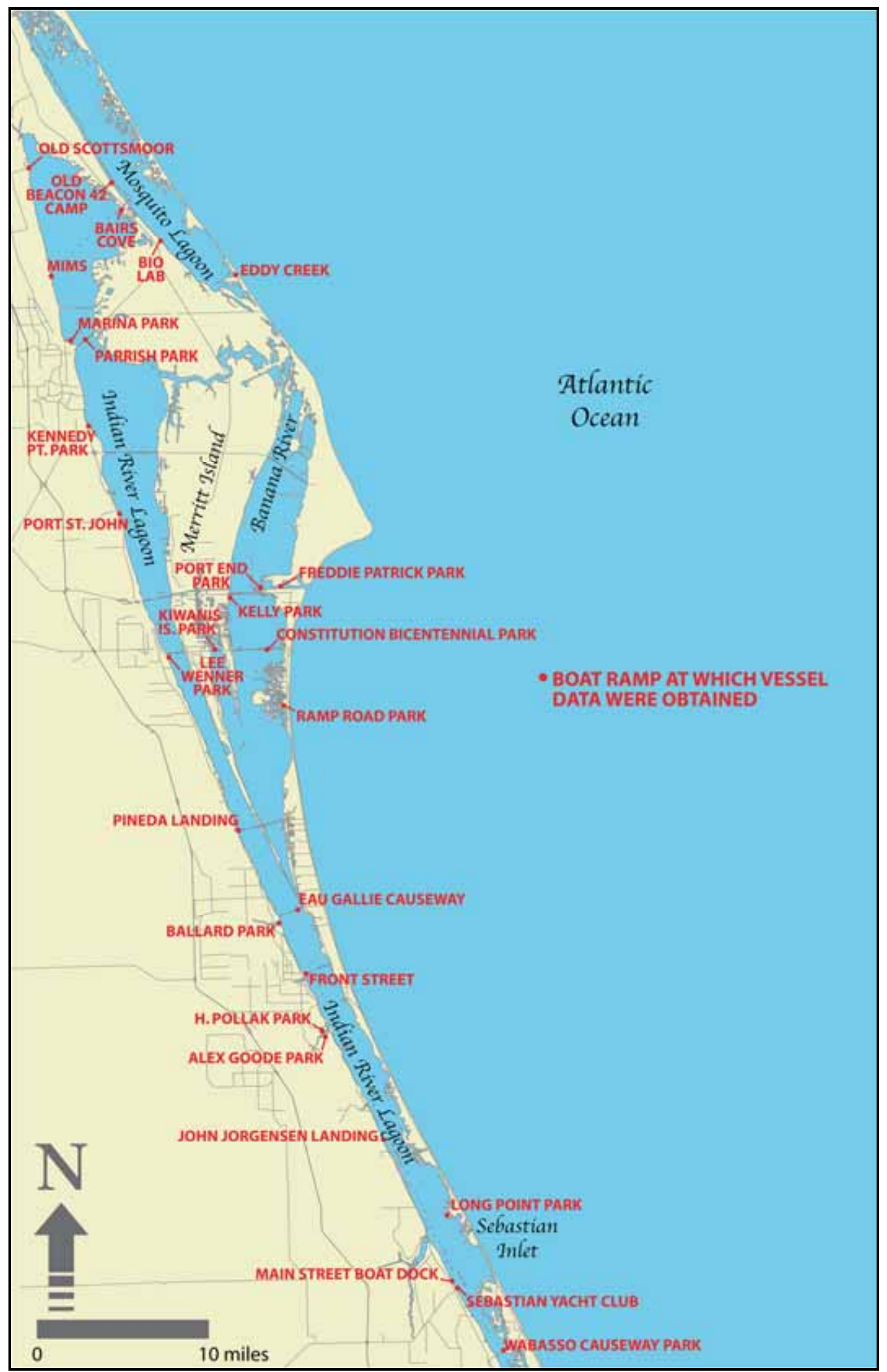

Figure 3. Brevard County Public Boat Ramps Surveyed. 
A sample of residential dock owners was selected by means of matching owner names and mailing addresses contained in the VTRS to waterfront parcel owner and address information obtained from Brevard County Property Tax records. Matches ensured that only those waterfront parcel owners who also owned boats were identified. A GIS 'select by location' analysis used a detailed shoreline to identify 16,295 waterfront properties from the Brevard County tax assessor's parcel database. A total of 5,989 VTRS records matched streets that corresponded to the selected Brevard County waterfront properties. A GIS database operation that evaluated owner last name, street name, and the mailing street number yielded 2,478 matches between the VTRS and waterfront parcels (Figure 4). An additional 29 records matched on the basis of owner last name and Post Office Box mailing addresses for a total of 2,507 VTRS / waterfront parcel matches. In addition, Florida Sea Grant personnel recorded the bow numbers from vessels berthed at docks associated with waterfront condominiums, yielding an additional 90 VTRS matches. A random sample of 2,000 private residences (residential and condominium) was selected from the 2,597 boats associated with waterfront condominiums and from parcel (street and post office box) to VTRS matches. 


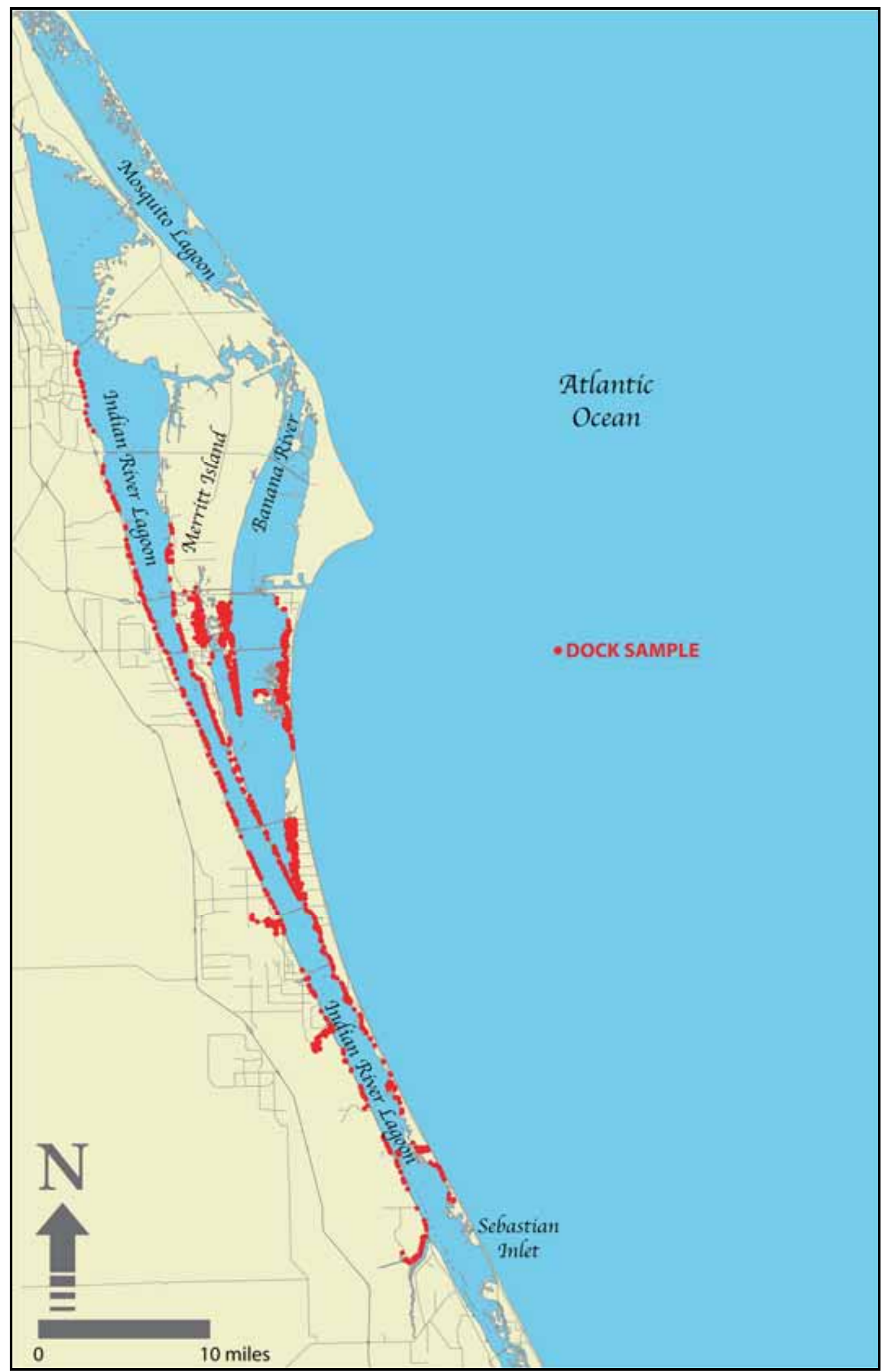

Figure 4. Spatial Distribution of the Brevard County Dock Sample. 


\section{Survey Return Breakdown}

Questionnaires were mailed in three waves, over a one-year period, to capture seasonal use patterns. Smart Mail Services Inc. validated boater addresses and conducted each mailing. A breakdown of survey mailings and returns for each survey wave is presented by waterway access group (i.e., marina (wet and dry storage), public ramp, and private dock) in Table 3. A total of 3,367 usable surveys were returned by June 1, 2007, which represented a 19\% overall return rate.

Table 3. Survey Return Breakdown.

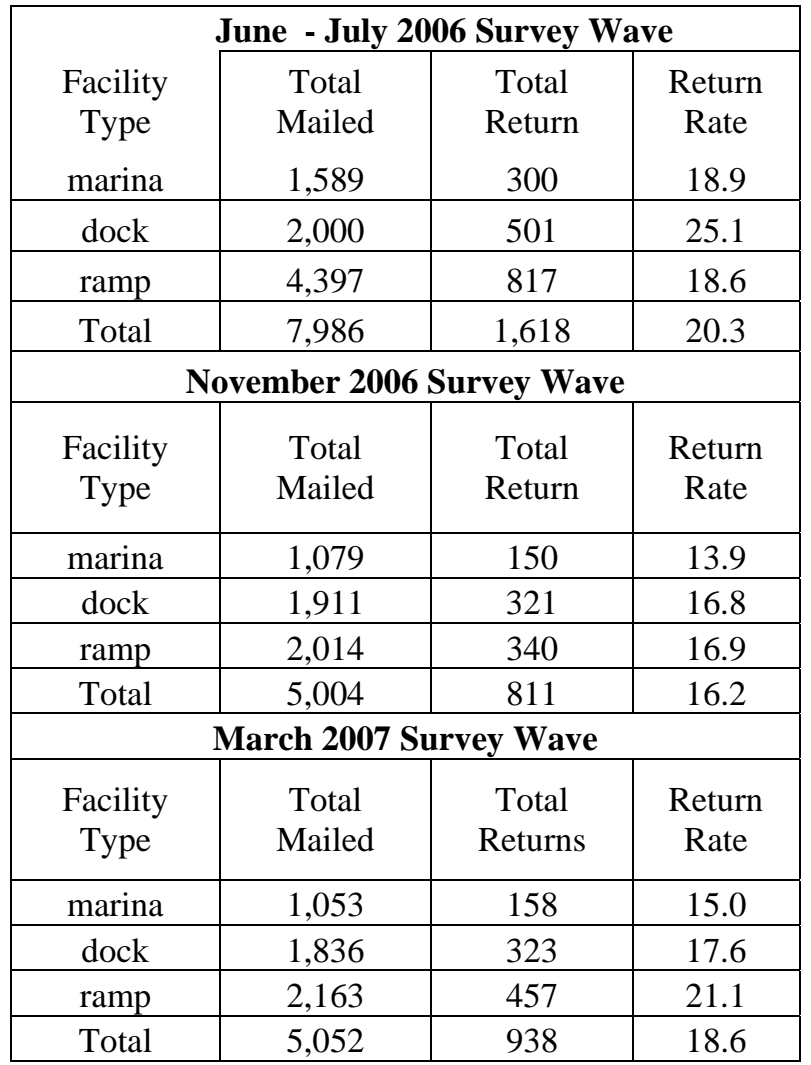




\section{Chapter 3. GIS Database Development}

\section{Spatial Database Design}

Questionnaire recipients were asked to (1) mark the start of their last two pleasure boating excursions on a map, (2) draw their entire travel routes, (3) identify their favorite boating destinations along those routes, and (4) annotate the map with abbreviations for the primary activities that they engaged in while at each destination. They were also asked to indicate by the letter " $C$ " any places on the map they considered to be congested. Data collected from 3,367 surveys over the three survey waves were digitized into the ESRI ArcGIS geographic information system (GIS). This translated to a sample of 6,307 trip departure sites and travel routes, 7,437 favorite boating destinations, and 2,907 locations of perceived congestion (Table 4).

Table 4. Trip Features Digitized from Returned Surveys.

\begin{tabular}{|l|c|c|c|c|}
\hline \multicolumn{1}{|c|}{ Trip Features } & Spring Mailing & Fall Mailing & Winter Mailing & Totals \\
\hline Origins (departure sites) & 3,105 & 1,611 & 1,591 & 6,307 \\
\hline Favorite Destinations & 3,854 & 1,846 & 1,737 & 7,437 \\
\hline Travel Routes & 3,105 & 1,611 & 1,591 & 6,307 \\
\hline Congested Spots & 2,026 & 388 & 493 & 2,907 \\
\hline
\end{tabular}

Spatial information was digitized 'on-screen' using a 1:24,000 scale shoreline, natural color Digital Orthophotograph Quarter Quadrangle (DOQQ) imagery, and the positions of marinas, ramps, navigation aids, and artificial reefs as background themes, to enhance the accuracy of digitized data. Trip departure sites and congested spots were digitized as point features, with each record coded with the survey control number and the trip number (i.e., first or second trip). Favorite destinations were digitized as point features and were coded with the survey control number, the trip number, and the activities that a respondent engaged in at each favorite destination. Travel routes were digitized as line features with the following attribute information coded: survey control number, trip number, and trip features such as one-way vs round, and whether or not the trip was confined to the study region.

The database structure allows information from survey questions to be 'linked' to digitized spatial information by the use of the survey control number (ID), which uniquely identified spatial and attribute information provided by each survey respondent. The selection and display of trip departure sites and favorite destination point data within the GIS is illustrated in Figure 5. A close-up of the Merritt Island boating area is displayed in the GIS view. Red dots represent departure sites identified by survey respondents; green dots represent favorite destinations; yellow dots represent a subset of favorite destinations where survey respondents reported that they like to "nature view." The 'Select by Attributes' window-upper left corner of Figure 5-illustrates a GIS database query that selects and displays those favorite destination points that are associated with nature viewing (e.g., NV = "Y”). The 'Selected Attributes of Destinations' window-lower left corner of Figure 5-displays all 'linked' database records in yellow. These records share the same survey control number (ID) that meet the query criterion of nature viewing $(\mathrm{NV})$. 
Reported travel routes within the Merritt Island boating region are displayed in Figure 6. Pink lines represent travel routes digitized from returned surveys; red and green dots illustrate departure sites and favorite destinations, respectively. The blue lines depicted in the GIS view represent two travel routes that were selected for display. The corresponding database records that are 'linked' to the two travel routes via the survey control number ID are highlighted blue in the ‘Attributes of Routes’ database window-lower left of Figure 6.

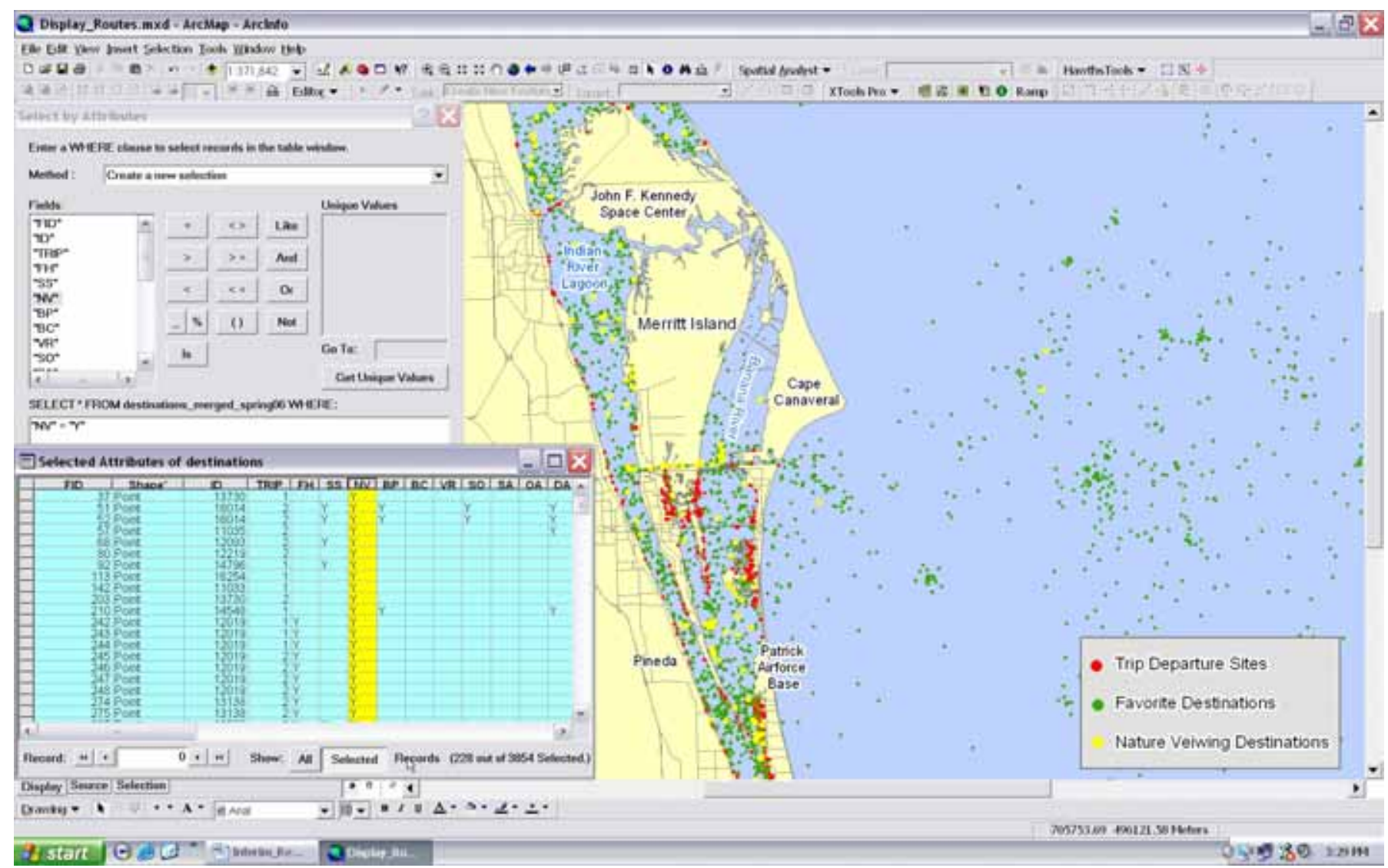

Figure 5. Example of GIS Attribute Query and Display: Trip Departure Sites and Favorite Destinations. 


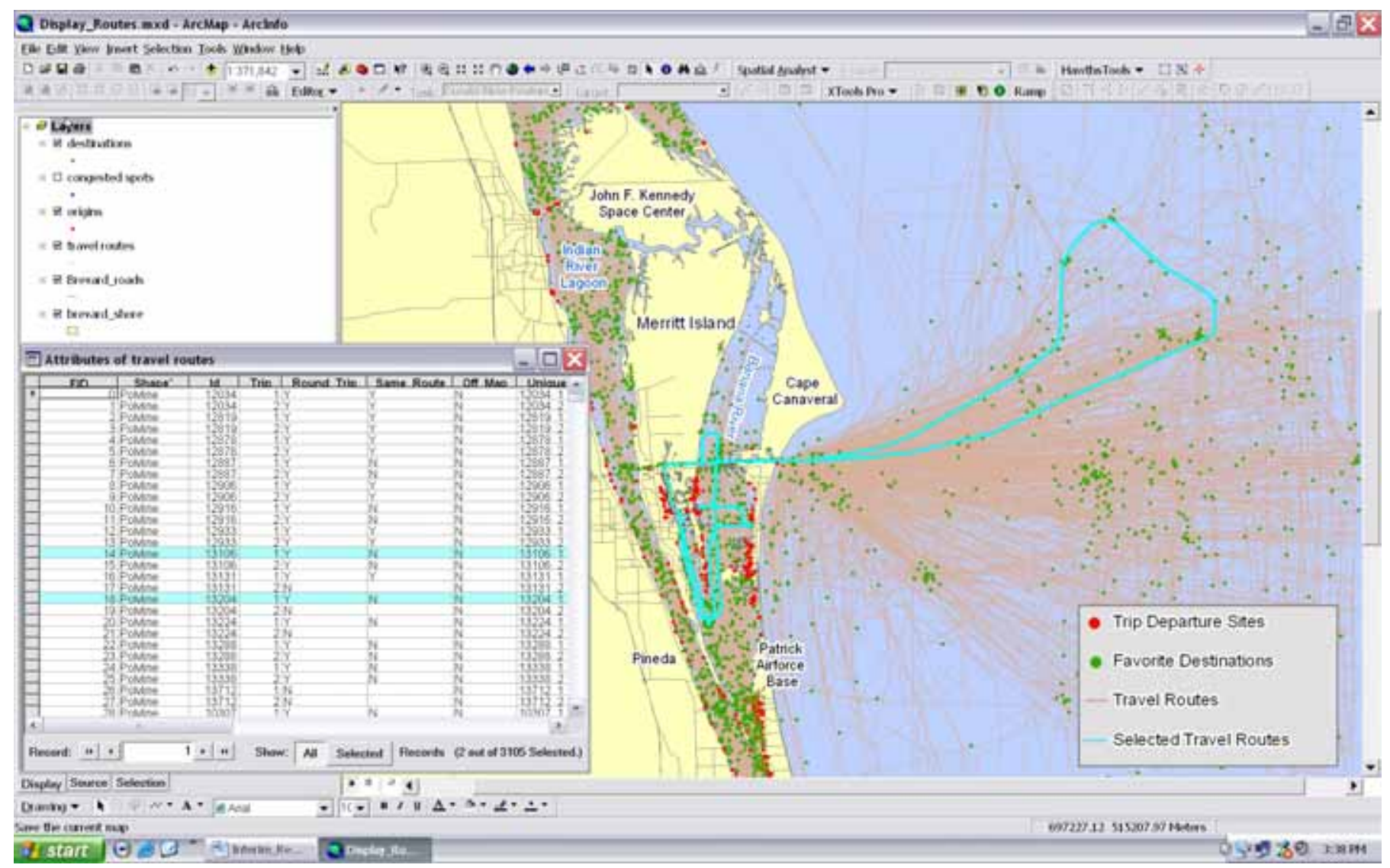

Figure 6. Example of GIS Attribute Query and Display: Reported Travel Routes. 


\section{Part 2- Summary Statistics}

\section{Chapter 4. Boater-Group Characteristics}

\section{Overview}

This chapter presents an evaluation and summary of responses to specific survey questions from boaters in the Brevard County study region. The sections of this chapter are divided according to themes that describe (1) vessel and boater profiles; (2) choice rationale for selecting departure sites and travel routes; (3) boating activities; and (4) perceived congestion. It should be noted that while questions were arranged to follow a logical progression on the survey instrument, the following results and associated discussion sections are arranged thematically and, therefore, questions do not necessarily follow the order in which they appeared on the survey instrument. The descriptive analysis presented in this chapter is based on information obtained from n=3,367 returned surveys, including both long and short versions of the questionnaire. The statistical analysis for some questions uses information from the first reported trip to reduce double counting - given that the vast percentage of respondents were consistent in their vessel use from trip 1 to trip 2. The number of survey respondents to specific survey questions varies from question to question, as does the sample size associated with the various user groups that responded to those questions. For convenience, the sample sizes are listed within each summary table and a copy of the survey instrument is provided in Appendix A.

\section{Vessel and Boater Profile: Access Type, Vessel Type, and Boating Experience}

- A breakdown of survey respondents is shown in Table 1. Of the $n=3,282$ survey respondents that answered Question 8, approximately 52\% accessed the water from a Boat Ramp. Those launching boats from Docks accounted for roughly 29\% of survey respondents. Together, these two groups accounted for over $80 \%$ of the survey respondents who answered Question 8. Boaters accessing the water from Marina Wet Slips accounted for about $14 \%$ of the survey participants, while those using Marina Dry Storage facilities accounted for slightly less than $4 \%$. Survey respondents associated with the Shoreline/Causeway access group accounted for less than $2 \%$ of sample (Table 1; Question 8). 
Table 1. Survey Response by Waterway Access Category.

\begin{tabular}{lccc}
\hline Access Category & $\begin{array}{c}\text { Frequency } \\
\text { Count }\end{array}$ & $\begin{array}{c}\text { Percentage } \\
\text { of total }\end{array}$ & Rank \\
Boat Ramp & $\mathbf{1 , 6 9 8}$ & $\mathbf{5 1 . 7 4 \%}$ & $\mathbf{1}$ \\
Dock & 950 & $28.94 \%$ & 2 \\
Shore/Causeway & 41 & $1.25 \%$ & 5 \\
Marina Wet Slip & 464 & $14.14 \%$ & 3 \\
Marina Dry Storage & 129 & $3.93 \%$ & 4 \\
& $\mathrm{n}=3,282$ respondents to Question 8 \\
\hline
\end{tabular}

- A summary of the vessels used by survey respondents is given in Table 2. Of the $n=3,281$ survey responses to Question 6 (and reported trip 1), 48.5\% of those respondents used boats that fell into the Open Fisherman category (which was the most common vessel category found amongst survey participants); followed by Sailboats with cabins at $12.4 \%$, Off-Shore Fishing Boats at $11.0 \%$, and Power Cruisers at 9.3\%. These four vessel types accounted for roughly $81 \%$ of all vessels used by survey respondents who reported trips. Cabin-less Sailboats and Speedboats of the Cigarette variety were associated with less than $1.0 \%$ of the trips reported by survey respondents (Table 2; Question 6).

Table 2. Vessel Used for Reported Trips.

\begin{tabular}{lrc}
\hline Vessel Type & $\begin{array}{c}\text { Frequency } \\
\text { Count }\end{array}$ & $\begin{array}{c}\text { Percentage } \\
\text { of Total }\end{array}$ \\
Jet Ski & 56 & $1.71 \%$ \\
Kayak/Row/Canoe & 41 & $1.25 \%$ \\
Sailboat (no Cabin) & 21 & $0.64 \%$ \\
Sailboat (with Cabin) & $\mathbf{4 0 8}$ & $\mathbf{1 2 . 4 3 \%}$ \\
Speed Boat (Runabout) & 253 & $7.71 \%$ \\
Speed Boat (Cigarette) & 21 & $0.64 \%$ \\
Open Fisherman & $\mathbf{1 , 5 9 1}$ & $\mathbf{4 8 . 5 0 \%}$ \\
Off-Shore Fisherman & $\mathbf{3 6 1}$ & $\mathbf{1 1 . 0 0 \%}$ \\
Power Cruiser & $\mathbf{3 0 6}$ & $\mathbf{9 . 3 3 \%}$ \\
Deck Boat & 66 & $2.01 \%$ \\
Pontoon & 110 & $3.35 \%$ \\
Other & 47 & $1.43 \%$ \\
& & \\
& $\mathrm{n}=3,281$ respondents to Question 6 \\
\hline
\end{tabular}


- Average (used interchangeably with “mean”) length and draft statistics for vessels used by survey respondents (Question 7) are shown in Table 3. The survey results revealed that the average vessel length was 31.8 feet for Marina Wet Slip users, followed by Marina Dry Storage and Dock users at 25.4 and 22.8 feet, respectively. Not surprisingly, the shortest vessel lengths were associated with survey respondents launching from Boat Ramps (18.8 feet) and Shoreline/Causeways (16.2 feet). In all cases, the median vessel length was very similar to the average vessel length indicating a fairly symmetrical distribution of values about their respective means.

- Similarly, the mean and median vessel drafts were highest for respondents departing from Marina Wet Slips (with a mean draft of 3.72 feet and a median of 4 feet), followed by users of Marina Dry Storage facilities (with a mean draft of 2.16 and median of 2.2 feet) and boaters departing from Docks (with a mean and median draft of 2 feet). As expected, the smallest vessel drafts were associated with respondents departing from Boat Ramps or the Shoreline/Causeway, with a mean vessel draft of approximately 1.2 feet. The median draft for Boat Ramp users was 1 foot, and the median draft for respondents departing from the Shoreline/Causeway was 6 inches.

- Based on the $n=3,253$ respondents to survey Question 7 (vessel length), it was shown that the mean vessel length was approximately 22 feet, with a median vessel length of 20 feet. Of the 3,080 respondents to Question 7 (draft), the mean vessel draft was shown to be 1.87 feet, and the median vessel draft was 1.5 feet (Table 3; Question 7).

Table 3. Mean Length and Draft by Departure/Water Access Category.

\begin{tabular}{|c|c|c|c|c|c|c|}
\hline \multirow[b]{2}{*}{ Access Category } & \multirow[b]{2}{*}{ Count } & \multicolumn{2}{|c|}{ Length (ft.) } & \multirow[b]{2}{*}{ Count } & \multicolumn{2}{|c|}{ Draft (ft.) } \\
\hline & & Mean & Median & & Mean & Median \\
\hline Boat Ramp & 1,673 & 18.8 & 18 & 1571 & 1.25 & 1.0 \\
\hline Dock & 938 & 22.8 & 21 & 887 & 2.00 & 2.0 \\
\hline Shore/Causeway & 39 & 16.2 & 16 & 34 & 1.15 & 0.5 \\
\hline Marina Wet Slip & 461 & 31.8 & 32 & 455 & 3.72 & 4.0 \\
\hline Marina Dry Storage & 129 & 25.4 & 25 & 120 & 2.16 & 2.2 \\
\hline Other & 13 & 31.8 & 32 & 13 & 3.20 & 3.0 \\
\hline All & 3,253 & 22.1 & 20 & 3,080 & 1.87 & 1.5 \\
\hline
\end{tabular}


- Summary statistics on the months that survey respondents reside in the state of Florida are shown in Table 4. The mean number of months per year that respondents reside in the state of Florida is approximately 11.8 months (based on information gathered from $n=2,442$ survey respondents). For all waterway access categories, the median number of months in residence is 12 months.

- The results suggest that the typical survey respondent departing from a Boat Ramp, Dock, Marina, or Shoreline/Causeway is essentially a year-round Florida resident based on the observed mean number of months and the estimated 95\% confidence intervals. This is especially true for respondents departing from Ramps-the user group with the highest average number of months in residence (11.93 months). Although there are only slight differences in the mean number of months in residence by category, Boat Ramp users had a reported mean number of months in residence that was significantly greater, in a statistical sense, than the overall average of 11.81 months at 95\% confidence (Table 4; Question 19).

Table 4. Mean Number of Months Resident in Florida by Water Access Category.

\begin{tabular}{|c|c|c|c|c|}
\hline Access Category & Count & Mean & $\mathbf{s}$ & $95 \%$ Confidence Interval \\
\hline Boat Ramp & 1,357 & 11.93* & 0.55 & $11.90-11.96$ \\
\hline Dock & 627 & 11.71 & 1.29 & $11.61-11.87$ \\
\hline Shore/Causeway & 31 & 12 & 0.0 & NA \\
\hline Marina Wet Slip & 330 & 11.62 & 1.57 & $11.44-11.79$ \\
\hline Marina Dry Storage & 86 & 11.52 & 1.79 & $11.13-11.90$ \\
\hline Other** & 11 & 8.90 & 4.52 & $5.94-11.87$ \\
\hline All & 2,442 & 11.81 & 1.08 & $11.76-11.85$ \\
\hline \multicolumn{5}{|c|}{$\mathrm{n}=2,442$ respondents to Question 19; } \\
\hline \multicolumn{5}{|c|}{$\begin{array}{l}\text { * denotes a mean value that is significantly higher than the overall average } \\
\text { value of } 11.81 \text { months at the } 95 \% \text { confidence level }\end{array}$} \\
\hline \multicolumn{5}{|c|}{ ** denotes a category with a small sample size } \\
\hline \multicolumn{5}{|c|}{$\mathrm{NA}=$ not applicable due to zero variance } \\
\hline Note: median $=1$ & hs for & rater & 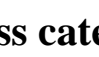 & ories \\
\hline
\end{tabular}


- Summary statistics for years boating experience of survey participants are shown in Table 5, and a graph of the distribution of values is shown in Figure 1. Survey respondents had, on average, approximately 19.6 years of recreational boating experience, with a median of 18 years boating experience. The most common answer among the $n=2,455$ survey respondents was 20 years boating experience (the mode).

- It was estimated that the mean number of years of boating experience among survey respondents was somewhere between 19.06 and 20.17 years overall, based on the estimated 95\% confidence interval for the mean. The maximum reported number of years boating experience was 90 years, and the minimum number was 0.1 years (Table 5; Question 20).

Table 5. Years Boating Experience in Florida (all survey respondents).

\begin{tabular}{lc}
\hline Statistic & Boating Experience (in years) \\
Mean & $\mathbf{1 9 . 6 1}$ \\
Standard Deviation & 14.02 \\
Minimum & 0.1 \\
Maximum & 90.0 \\
Median & 18 \\
Mode & 20 \\
95\% Confidence Interval & $19.06-20.17$ \\
$\mathrm{n}=2,455$ survey respondents (Question 20$)$ \\
\hline
\end{tabular}

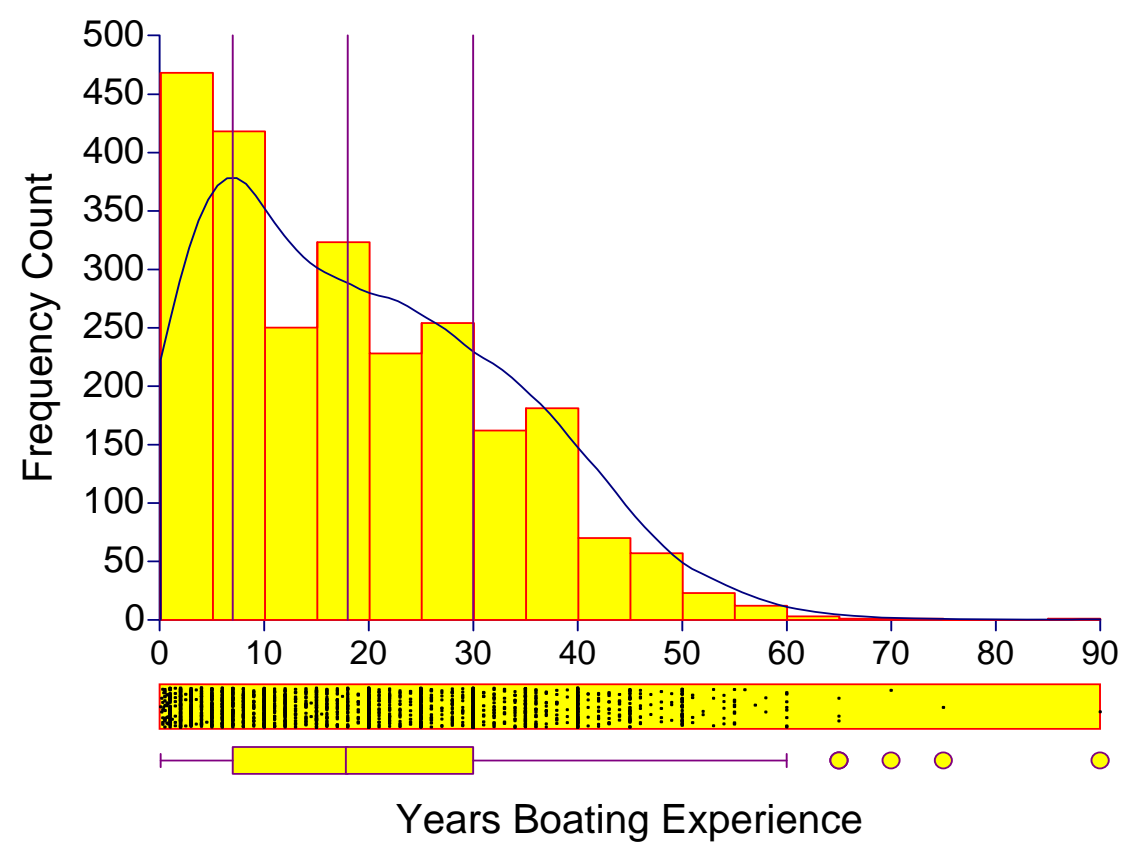

Figure 1. Histogram and Box Plot of Years Boating Experience (in years). 
- Survey respondents that accessed the water from Docks and Boat Ramps tended to have the greatest amount of boating experience with 21.9 and 19.3 years of boating experience, on average, respectively. All remaining water access groups had an average number of years boating experience that fell significantly below 19 years and median values that were less than the overall median of 18 years.

- Boaters departing from Marina Wet Slips tended to have the least amount of boating experience (16.4 years). Moreover, Marina Wet Slips users who participated in the survey tended to have about three years less boating experience than boaters launching from Boat Ramps, and roughly 5.5 years less boating experience than boaters departing from Docks (See Tables 5 and 6; Question 20).

Table 6. Years of Boating Experience in Florida by Waterway Access Category.

\begin{tabular}{|c|c|c|c|c|c|c|}
\hline \multirow[b]{2}{*}{ Access Category } & \multirow[b]{2}{*}{ n } & \multicolumn{4}{|c|}{ (in years) } & \multirow[b]{2}{*}{ Max } \\
\hline & & Mean & s dev. & Median & Min & \\
\hline Boat Ramp & 1,358 & 19.3 & 14.1 & 18 & .1 & 90 \\
\hline Shore/Causeway & 30 & $17.3^{*}$ & 15.9 & 10 & 2.0 & 60 \\
\hline Marina Wet Slip & 332 & $16.4^{*}$ & 13.8 & 13 & .5 & 58 \\
\hline Marina Dry Storage & 87 & $18.8 *$ & 13.3 & 16 & .7 & 50 \\
\hline Dock & 627 & 21.9 & 13.4 & 20 & .4 & 75 \\
\hline Other & $11^{* *}$ & 18.1 & 14.7 & 15 & 1.0 & 45 \\
\hline \multicolumn{7}{|c|}{$\mathrm{n}=2,455$ survey respondents (Question 20) } \\
\hline
\end{tabular}


- Statistics on the percentage of survey respondents that have completed a boater safety or seamanship course are given in Table 7 . Roughly $65 \%$ of the $n=2,439$ survey respondents indicated that they had had a boating safety or seamanship course.

- Boaters launching from either the Shoreline/Causeway or from Boat Ramps tended to be the least likely to have had a boating safety or seamanship course.

- Survey respondents accessing the water from Marina Wet Slips were the most likely to have had a boating safety or seamanship course (82.1\%); followed by boaters departing from Marina Dry Storage facilities (77.4\%) and Docks (72.7\%) - (Table 7; Question 21).

Table 7. Boaters Having Completed a Boat Safety/Seamanship Course By Waterway Access Category.

\begin{tabular}{lrrrc}
\hline Access Category & $\mathbf{n}$ & Yes & Percentage & Above Average? \\
Boat Ramp & 1355 & 772 & $56.9 \%$ & No \\
Shoreline/Causeway & 31 & 11 & $35.4 \%$ & No \\
Marina Wet Slip & $\mathbf{3 3 0}$ & $\mathbf{2 7 1}$ & $\mathbf{8 2 . 1 \%}$ & Yes \\
Marina Dry-Storage & $\mathbf{8 5}$ & $\mathbf{6 6}$ & $\mathbf{7 7 . 4 \%}$ & Yes \\
Dock & $\mathbf{6 2 7}$ & $\mathbf{4 5 6}$ & $\mathbf{7 2 . 7 \%}$ & Yes \\
Other & $11 *$ & 9 & $81.8 \%$ & NA \\
Overall & & & & \\
\hline
\end{tabular}

*Too small of a sample size to statistically evaluate 
- A breakdown of the age of survey participants by user group is given in Table 8 . The distribution of age for the $n=2,425$ respondents to Question 22 is illustrated in Figure 2. Survey respondents were, on average, 54 years of age.

- Respondents that accessed the water from Marinas Wet Slips and Marina Dry Storage facilities tended to be about 5 years older than the average survey respondent; and those departing from private Docks were about 4 years older than the average respondent. The average age of respondents in these three categories was significantly higher than the average value of 54.1 years at the $95 \%$ confidence level. The median age of survey respondents departing from Marinas was 60 years, whereas the median age of respondents departing from Docks was 57 years.

- Survey respondents launching from Public Boat Ramps tended to be about 4 years younger than the average respondent, and about 9 years younger than respondents departing from Marina Wet Slip and Marina Dry Storage facilities. The average age of survey respondents departing from Boat Ramps (50.4 years) and from the Shoreline/Causeway (52.1 years) tended to be significantly less than the average age of all survey respondents (54.1 years). The median age of survey respondents launching from Boat Ramps was the lowest (50 years), a value that was less than the overall median age of all survey respondents by three years (Table 8; Question 22).

Table 8. Age of Boaters by Waterway Access Category.

\begin{tabular}{lrrrrrr}
\hline & & \multicolumn{5}{c}{ (in years) } \\
Access Category & $\mathbf{n}$ & Mean & Std. Dev. & Median & Min & Max \\
& & & & & & \\
Boat Ramp & 1,351 & 50.4 & 11.6 & 50 & 18 & 88 \\
Shoreline/Causeway & 31 & 52.1 & 13.6 & 53 & 22 & 82 \\
Marina Wet-Slip & $\mathbf{3 2 6}$ & $\mathbf{5 9 . 5 *}$ & $\mathbf{1 1 . 1}$ & $\mathbf{6 0}$ & $\mathbf{2 5}$ & $\mathbf{9 2}$ \\
Marina Dry Storage & $\mathbf{8 6}$ & $\mathbf{5 9 . 0}$ & $\mathbf{1 3 . 2}$ & $\mathbf{6 0}$ & $\mathbf{2 8}$ & $\mathbf{8 9}$ \\
Dock & $\mathbf{6 2 0}$ & $\mathbf{5 8 . 4 *}$ & $\mathbf{1 2 . 5}$ & $\mathbf{5 7}$ & $\mathbf{1 4}$ & $\mathbf{9 0}$ \\
Other & $11^{* *}$ & 59.0 & 13.4 & 58 & 36 & 84 \\
Overall & & & & & & \\
& $\mathrm{n}=2,425$ & 54.1 & 12.5 & 53 & 14 & 92 \\
\hline
\end{tabular}

* Denotes above-average value; ** Small sample (not able to statistically evaluate) 


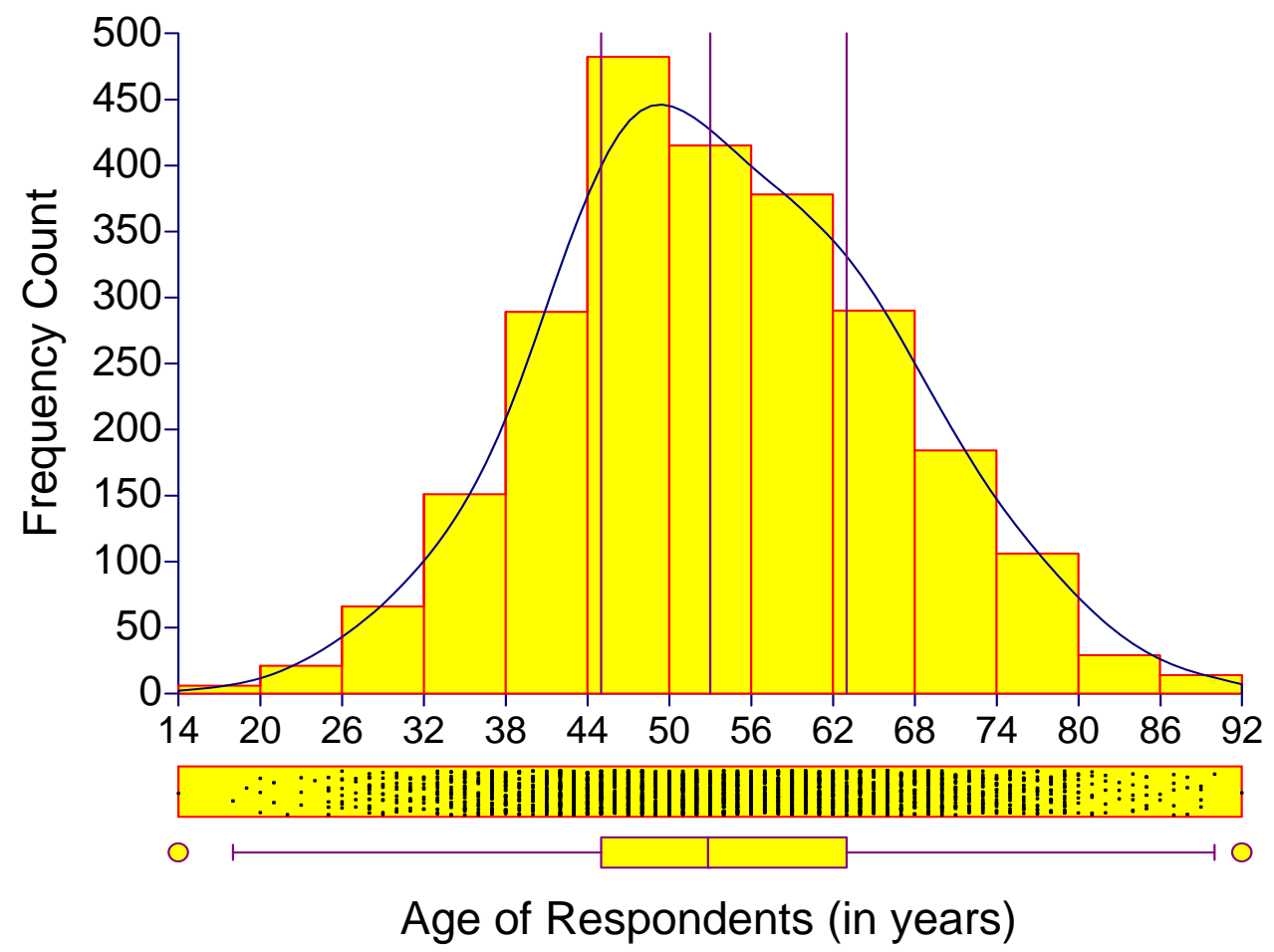

Figure 2. Histogram and Box Plot Showing the Age of Survey Participants. 
- $\quad$ Statistics on Drive Time from home to departure site are shown in Table 9 for respondents launching their boats from Boat Ramps, or departing from Marina Wet Slips, and Marina Dry Storage Facilities. The distribution of drive-time values is illustrated in Figure 3. On average, survey respondents launching from either a Boat Ramp or departing from a Marina were shown to have drive times of approximately 40 minutes from their home. The distribution of drive-time values shown in Figure 3 (for respondents traveling three hours or less) shows that most respondents travel 20 minutes or less to their departure sites. Nevertheless, there is a large contingent of respondents - about 33\% of survey participants - that travel over fairly great distances (in excess of 40 minutes) to gain access to their departure locations.

- Survey respondents departing from Boat Ramps tended to travel an average of 39 minutes from home to their launch site, a value that is not significantly different from the typical respondent's drive time for the three user categories examined. Note that the maximum reported drive-time distance was 1,200 minutes or 20 hours.

- $\quad$ Survey respondents departing from Marina Wet Slips were shown to travel approximately 36 minutes, on average, from their home to the Marinas from which they departed; with a median drive time of roughly 20 minutes. The difference in the mean and median drive time values for this water access category is attributable to the fact that the distribution of drive-time values is highly (positively) skewed (i.e., likely due to the presence of extreme or outlying values). The presence of large outlying values also accounts for the relatively large standard deviation associated with this category of respondents.

- Note that the reported maximum drive time from home to Marina Wet Slips was 1,800 minutes or 30 hours. All in all, Marina Wet Slip users tend to have drive times that are somewhere between 28 and 45 minutes based on a 95\% confidence interval.

- Respondents departing from Marina Dry Storage facilities tended to travel, on average, just over 32 minutes from their homes to the facilities from which they launched their boats. Note that the average drive time from home to Marina Dry Storage facilities (32.5 minutes) was significantly less than the overall average for the three water access categories examined in this section (38.9 minutes).

- Note that the reported maximum drive-time distance from home to Marina Dry storage facilities was 105 minutes (or 1 hour and 45 minutes). On average, respondents departing from Marina Dry Storage facilities tend to travel between 29 and 36 minutes from home to their departure sites based on the estimated 95\% confidence interval (Table 9; Question 10). 
Table 9. Drive Time from Home to Departure Site for Reported Trips (in minutes).

\begin{tabular}{|c|c|c|c|c|c|c|}
\hline \multirow[b]{2}{*}{ Access Category } & \multirow[b]{2}{*}{$\mathbf{n}$} & \multicolumn{4}{|c|}{ (in minutes) } & \multirow[b]{2}{*}{ 95\% Confidence Interval } \\
\hline & & Mean & Std. Dev. & Median & Max & \\
\hline Boat Ramp & 2,895 & 39.6 & 51.3 & 30 & 1200 & $37.7-41.5$ \\
\hline Marina Wet-Slip & 625 & 36.2 & 105.4 & 20 & 1800 & $27.8-44.4$ \\
\hline Marina Dry Storage & 176 & $32.5^{*}$ & 22.3 & 27.5 & 105 & $29.2-35.8$ \\
\hline Overall & $n=3,696$ & 38.9 & 64.3 & 30 & 1800 & $36.8-41.0$ \\
\hline
\end{tabular}

* mean is significantly less than average in comparison to overall category at the $95 \%$ confidence level.

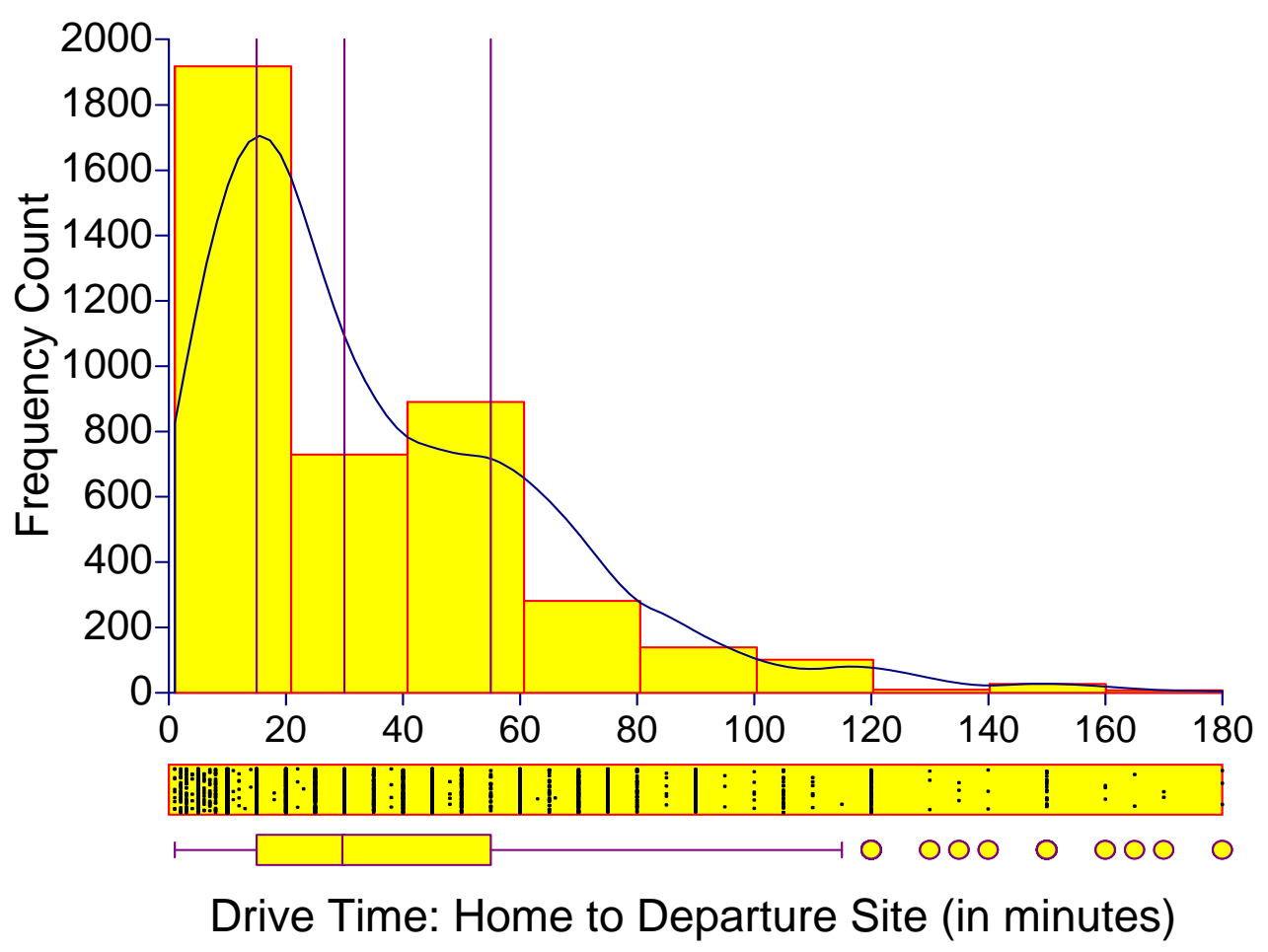

Figure 3. Histogram and Box Plot of Drive Times for Boaters Traveling Three Hours or Less to their Departure Sites. 
- $\quad$ Statistics on departure times are shown in Table 10. Note that the average AM and PM start times for trips reported by survey respondents were highly variable amongst the various waterway access categories.

- Survey respondents departing from public Boat Ramps were shown to launch earlier than their counterparts during the morning hours (with an average start time of 7:21AM); and tended to depart later during the afternoon hours (around 3:30PM). In general, Boat Ramp users had AM start times that were significantly earlier than the average start time of 7:51AM; and PM start times that were significantly later than the average of 2:35PM.

- On average, survey respondents departing from Docks and Marina Wet Slip users tended to begin their trips at approximately 8:31AM and 8:54AM, respectively-start times that were significantly later than the overall average start time of 7:51AM. Marina Wet Slip and Dock users tended to have average PM departure times of around 2:15PM (a value that was very close to the overall average PM start time of 2:35PM).

- Survey respondents departing from Marina Dry Storage facilities started their morning trips at 8:00AM (a start time that is not significantly different from the overall average of 7:51AM). Note, however, that the average PM launch time for Marina Dry Storage users was $1: 36 \mathrm{PM}$-indicating a start time that is significantly earlier than the overall afternoon average of 2:35PM (Table 10; Question 2, AM and PM).

Table 10. Average Departure Time by Waterway Access Category.

\begin{tabular}{|c|c|c|c|c|c|c|}
\hline \multirow[b]{2}{*}{ Access Category } & \multicolumn{6}{|c|}{ Average Value } \\
\hline & $\mathbf{n}$ & $\begin{array}{l}\text { AM } \\
\text { Hour }\end{array}$ & $\begin{array}{l}\text { AM } \\
\text { Time }\end{array}$ & $\mathbf{n}$ & $\begin{array}{l}\text { PM } \\
\text { Hour }\end{array}$ & $\begin{array}{l}\text { PM } \\
\text { Time }\end{array}$ \\
\hline Boat Ramp & 2,951 & 7.36 & $7: 21^{*}$ & 299 & 3.50 & $3: 30 * *$ \\
\hline Shoreline/Causeway & 63 & 7.59 & $7: 35$ & 12 & 2.00 & $2: 00$ \\
\hline Marina Wet Slips & 619 & 8.91 & $8: 54 * *$ & 224 & 2.21 & $2: 12$ \\
\hline Marina Dry Storage & 217 & 7.99 & $8: 00$ & 20 & 1.60 & $1: 36^{*}$ \\
\hline Dock & 1,119 & 8.52 & $8: 31^{* *}$ & 550 & 2.28 & $2: 17$ \\
\hline Other & 18 & 8.13 & 8:08 & 5 & 4.30 & $4: 18^{* *}$ \\
\hline All Groups & $\mathrm{n}=4,987$ & 7.86 & $7: 51$ & 1,110 & 2.59 & $2: 35$ \\
\hline
\end{tabular}

* Denotes a trip departure time that is significantly earlier than the average start time for all waterway access groups (at the 95\% confidence level).

** Denotes a trip departure time that is significantly later than the average start time for all waterway access groups (at the 95\% confidence level). 
- On-water trip duration statistics by user group are shown in Table 11 for trips that are equal to or less than 24 hours; i.e., for trips characterized as "day trips.”

- Survey respondents launching from Marina Dry Storage facilities and Boat Ramps tend to spend more time on the water than those accessing the water from the other launch departure categories for trips of 24 hours or less in duration. Respondents departing from Boat Ramps were shown to have a mean day-trip duration of a little over 6 hours, while those departing from Marina Dray Storage facilities had an average reported day-trip duration of a little less than 7 hours.

- Boaters departing from private Docks were shown to have a mean day-trip duration of a little less than 5 hours, which was well below the average day-trip duration of survey respondents associated with other waterway access categories (Table 11; Question 3).

Table 11. Trip Duration: On-Water Travel Time by Waterway Access Category (All Reported Day Trips; Trip duration $\leq 24$ hours).

\begin{tabular}{|c|c|c|c|c|}
\hline \multirow[b]{2}{*}{ Access Category } & \multicolumn{4}{|c|}{ (in hours) } \\
\hline & $\mathbf{n}$ & Mean & 95\% C.I. & Median \\
\hline Boat Ramp & 3,083 & $6.15 *$ & 6.06-6.24 & 6 \\
\hline Shoreline/Causeway & 75 & 5.48 & $4.69-6.28$ & 5 \\
\hline Marina Wet Slip & 637 & 5.84 & $5.50-6.18$ & 5 \\
\hline Marina Dry Storage & 229 & $6.48 *$ & 6.10-6.87 & 6 \\
\hline Dock & 1,682 & $4.78 * *$ & 4.62-4.93 & 4 \\
\hline Other & 17 & 6.73 & $4.91-8.55$ & 6 \\
\hline Overall & $\mathrm{n}=5,723$ & 5.72 & $5.63-5.80$ & 5 \\
\hline
\end{tabular}


- On-water trip duration statistics by user group are shown in Table 12 for trips that are greater than 24 hours; i.e., for trips characterized as “overnighters.”

- $\quad$ Based on the reported trips by survey respondents, boaters departing from Marina Wet Slips tended to spend more time on the water than those departing from other waterway access sites, for trips that exceeded 24 hours in duration and were less than 168 hours. Survey respondents departing from Marina Wet Slips reported overnight trip durations that averaged 63.7 hours (or roughly 2.7 days).

- The average reported overnight trip for all waterway access categories was approximately 53.1 hours in duration (or 2.2 days), and the median overnight trip duration was 48 hours (or 2 days).

- Survey respondents leaving from private Docks reported overnight trips of 55.9 hours in duration - a value that was not significantly different from the overall average overnight trip duration of 53.1 hours at the $95 \%$ confidence level.

- Overnight trips reported by boaters launching from both Ramps and Marina Dry Storage facilities were approximately 42 hours in duration (or about 1.75 days). The average reported overnight trip duration for both Ramp and Marina Dry Storage facility users was found to be significantly less than the overall average trip duration of 53.1 hours or 2.2 days (Table 12; Question 3).

Table 12. Trip Duration: On-Water Travel Time by Waterway Access Category (All Reported Overnight Trips; Trip duration $>24$ hours and $<168$ hours).

\begin{tabular}{|c|c|c|c|c|}
\hline \multirow[b]{2}{*}{ Access Category } & \multicolumn{4}{|c|}{ (in hours) } \\
\hline & $\mathbf{n}$ & Mean & 95\% C.I. & Median \\
\hline Boat Ramp & 240 & $41.8 * *$ & $38.9-44.6$ & 32 \\
\hline Shoreline/Causeway & $3 \dagger$ & 56.0 & NA & 72 \\
\hline Marina Wet Slip & 225 & $63.7 *$ & $59.4-68.0$ & 48 \\
\hline Marina Dry Storage & 21 & $42.8^{* *}$ & $32.8-52.8$ & 34 \\
\hline Dock & 155 & 55.9 & $50.7-61.1$ & 48 \\
\hline Other & $5 \dagger$ & 74.0 & NA & 72 \\
\hline Overall & $\mathrm{n}=649$ & 53.1 & $50.7-55.4$ & 48 \\
\hline \multicolumn{5}{|c|}{$\begin{array}{l}\text { * Denotes an on-water overnight travel time/trip duration that is significantly greater than } \\
\text { the average of } 53.1 \text { hours at the } 95 \% \text { confidence interval. } \\
\text { ** Denotes an on-water overnight travel time/trip duration that is significantly less than } \\
\text { the average of } 53.1 \text { hours at the } 95 \% \text { confidence interval. }\end{array}$} \\
\hline
\end{tabular}


- Statistics for weekday vs. weekend trips are shown in Table 13. Of the $n=6,359$ trips reported by survey respondents, approximately 41.5\% were weekday trips (Monday through Friday) and 58.5\% were weekend trips (Saturday or Sunday).

- Survey respondents launching from Boat Ramps or the Shoreline/Causeway tended to have a lower percentage of weekday trips and a higher percentage of weekend trips in comparison to the overall average or respondents from other water access categories. Almost two-thirds of reported trips by Ramp users took place on weekend days.

- A greater percentage of trips reported by respondents departing from Marina Dry Storage facilities took place during weekdays. Respondents from this water access category reported that $52 \%$ of trips took place during the weekdays, whereas $48 \%$ of their trips took place during the weekend.

- Adjusting for the number of weekday vs. weekend days (5 vs. 2 days), the typical weekend day (Saturday or Sunday) was associated with 3.5 times more reported trips per day than the typical weekday (Monday through Friday)—see Relative Impact Score (Table 13; Question 4).

Table 13. Weekday vs. Weekend Trips by Water Access Category.

\begin{tabular}{|c|c|c|c|c|c|}
\hline \multirow[b]{2}{*}{ Access Category } & \multirow[b]{2}{*}{$\mathbf{n}$} & \multicolumn{4}{|c|}{ Trips and Percentages } \\
\hline & & Weekday & $\%$ & Weekend & $\%$ \\
\hline Boat Ramp & 3,317 & 1,259 & $37.95 * *$ & 2,058 & $62.05^{*}$ \\
\hline Shoreline/Causeway & 75 & 17 & $22.66^{* *}$ & 58 & $77.34 *$ \\
\hline Marina Wet Slip & 874 & 417 & 47.71 & 457 & 52.29 \\
\hline Marina Dry Storage & 248 & 129 & $52.02 *$ & 119 & $47.98 * *$ \\
\hline Dock & 1,820 & 802 & 44.07 & 1,018 & 55.93 \\
\hline Other† & 25 & 13 & 52.00 & 12 & 48.00 \\
\hline Overall & $\mathrm{n}=6,359$ & 2,637 & 41.47 & 3,722 & 58.53 \\
\hline
\end{tabular}

Adjusted Percentages reflecting the impact of a typical weekday vs. weekend day

Daily impact $\%$ weekday = weekday $\%$ divided by 5 days

Daily impact \% weekend = weekend \% divided by 2 days

Typical Weekday: 8.294; Typical Weekend: 29.265

Relative Impact Score: RIS = Daily Impact \% (Weekend) $/$ Daily Impact \% (Weekday)

$$
=29.3 / 8.3=3.5
$$

RSI $\rightarrow$ The typical weekend day is associated with approx. 3.5 times the number of typical weekday trips.

\footnotetext{
* Denotes a percentage value that is significantly greater than the overall average $\%$ at $95 \%$ Confidence.

** Denotes a percentage value that is significantly less than the overall average $\%$ at $95 \%$ Confidence.

$\dagger$ Small sample (not able to statistically evaluate)

Question 4: Q4_1_D - Day that trip 1 took place (1 - 7 = Monday - Sunday; period - blank) Q4_2_D - Day that trip 2 took place $(1-7$ = Monday - Sunday; period - blank $)$
} 


\section{Rationale for Selecting Departure Sites and Travel Routes}

This section characterizes the choice rationale for selecting departure sites (i.e., marina, public ramp), and waterway travel routes.

- Based on the ranked average response by rationale category, survey respondents preferred departure sites that had (a) adequate, safe, and secure parking; (b) sites that provided ease of launching and retrieving their boats and a short wait to launch; (c) proximity to their favorite on-water boating spots/destinations; (d) well-marked access channels at or near the launch site; and (e) launch sites that had no parking fees (Table 14; Question 11 criteria 1-14).

Table 14. Reasons for Selecting a Favorite Departure/Launch Site.

\begin{tabular}{|c|c|c|c|}
\hline \multirow{2}{*}{ Reason/Description } & \multirow[b]{2}{*}{ Count (n) } & \multicolumn{2}{|c|}{ Response* } \\
\hline & & Average & Rank $^{* *}$ \\
\hline 1 Deep-water access & 1813 & 2.30 & \\
\hline 2 Availability of restrooms & 1783 & 2.63 & \\
\hline 3 No parking / launch fee & 1796 & 1.99 & 7 \\
\hline 4 Well-marked access channels & 1821 & 1.96 & 6 \\
\hline 5 Proximity to favorite boating spots & 1833 & 1.80 & 4 \\
\hline 6 Adequate parking & 1841 & 1.52 & 2 \\
\hline 7 Availability of fishing supplies & 1769 & 3.01 & \\
\hline 8 Short wait to launch & 1748 & 1.91 & 5 \\
\hline 9 Gas, pump-out, maintenance service & 1780 & 3.36 & \\
\hline 10 Nearby amenities (e.g. restaurants) & 1742 & 3.40 & \\
\hline 11 Proximity to home & 1858 & 2.13 & \\
\hline 12 Ease of launching/retrieving boat & 1800 & 1.61 & 3 \\
\hline 13 Safe and secure parking & 1860 & 1.51 & 1 \\
\hline 14 Other reason: mixed (not ranked) & 289 & 1.50 & \\
\hline \multicolumn{4}{|c|}{ Based on $n=2,338$ respondents to Question 11} \\
\hline \multicolumn{4}{|c|}{$\begin{array}{l}\text { * Average response based on Key below; } \\
\text { ** Ranking: from “most important” to “least important” (reasons 1-13 only) } \\
\text { Note: Top-7 ranked categories highlighted in bold (excluding category 14). }\end{array}$} \\
\hline $\begin{array}{l}\text { Key: } \\
1 \text { - strongly agree (very important) } \\
2 \text { - agree (important) } \\
3 \text { - neutral } \\
4 \text { - disagree (somewhat unimportant) } \\
5 \text { - strongly disagree (very unimportant) }\end{array}$ & & & \\
\hline
\end{tabular}


- Top reasons for selecting a favorite travel route, based on $n=2,338$ survey respondents to Question 14 included (a) avoidance of congested areas; (b) enjoy scenic beauty; (c) quick access to favorite on-water boating spots/destination; (d) good fishing; (e) preference for well-marked channels and calm protected waters; and (f) avoidance of shallow water (Table 15; Question 14 criteria 1-11).

Table 15. Reasons for Selecting a Favorite Travel Route.

\section{Reason/Description}

1 Avoid congested areas

2 Avoid shallow water

3 Good fishing

4 Prefer well-marked channels

5 Prefer calm protected waters

6 Avoid speed

7 None are important - just cruise around

8 Easy access to supplies or fuel

9 Quick access to favorite boating spots

10 Enjoy scenic beauty

11 Other reason: mixed (not ranked)

\begin{tabular}{ccc} 
& \multicolumn{2}{c}{ Response* } \\
& -------- \\
Count (n) & Average & Rank** \\
& & \\
$\mathbf{2 3 3 7}$ & $\mathbf{1 . 7 2}$ & $\mathbf{1}$ \\
$\mathbf{2 3 3 8}$ & $\mathbf{2 . 3 9}$ & $\mathbf{7}$ \\
$\mathbf{2 3 1 1}$ & $\mathbf{2 . 0 3}$ & $\mathbf{4}$ \\
$\mathbf{2 3 2 5}$ & $\mathbf{2 . 0 4}$ & $\mathbf{5}$ \\
$\mathbf{2 3 1 9}$ & $\mathbf{2 . 3 0}$ & $\mathbf{6}$ \\
2263 & 2.38 & \\
2101 & 3.61 & \\
2217 & 3.13 & \\
$\mathbf{2 2 6 8}$ & $\mathbf{2 . 0 2}$ & $\mathbf{3}$ \\
$\mathbf{2 3 2 7}$ & $\mathbf{1 . 7 7}$ & $\mathbf{2}$ \\
251 & 1.61 &
\end{tabular}

Based on $n=2,338$ respondents to Question 14

\footnotetext{
* Average response based on Key below;

Key:

1 - strongly agree (very important)

2 - agree (important)

3 - neutral

4 - disagree (somewhat unimportant)

5 - strongly disagree (very unimportant)
}

** Ranking: from “most important” to "least important” (reasons 1-10 only)

Note: Top-7 ranked categories highlighted in bold (excluding category 11). 


\section{Boater Activity Profile}

A description of the recreational boating activities reported by $n=3,446$ survey respondents to Question 16 is presented in this section, as well as a ranking of chosen activities. Respondents were asked to choose, from an activity list provided in the survey, all of the activities in which they engage on a "typical" pleasure boating trip. The column labeled 'Count' is, therefore, equal to the total number of times a given activity was chosen by survey respondents. Note that many respondents selected multiple activities from the list hence, percentages will sum to greater than $100 \%$. The topfive activities (by rank) are highlighted in each table.

- Fishing ranked as the leading activity with approximately 53\% of survey respondents indicating that they engaged in this activity during a typical boating trip.

- Cruising was the second-most selected activity with a percentage of 33\%, followed closely by Nature Viewing at 32\%.

- Sightseeing and Socializing were activities that also commonly took place during a typical trip, with percentages of approximately 26\% and 23\%, respectively (Table 16; Question 14).

Table 16. Boaters’ Activity Statistics (entire sample/all respondents).

\begin{tabular}{lrcc}
\hline Activity & Count & $\begin{array}{r}\text { Percentage of } \\
\text { Respondents }\end{array}$ & Rank \\
Beach Picnicking & 539 & $15.6 \%$ & \\
Nature Viewing & $\mathbf{1 1 0 6}$ & $\mathbf{3 2 . 1 \%}$ & $\mathbf{3}$ \\
Sightseeing & $\mathbf{9 1 1}$ & $\mathbf{2 6 . 4 \%}$ & $\mathbf{4}$ \\
Cruising & $\mathbf{1 1 4 6}$ & $\mathbf{3 3 . 2 \%}$ & $\mathbf{2}$ \\
Daytime Anchoring & 566 & $16.4 \%$ & \\
Socializing & $\mathbf{8 1 5}$ & $\mathbf{2 3 . 6 \%}$ & $\mathbf{5}$ \\
Diving & 301 & $8.7 \%$ & \\
Overnight Anchoring & 371 & $10.7 \%$ & \\
Visit Restaurants & 607 & $17.6 \%$ & \\
Fishing & $\mathbf{1 8 3 4}$ & $\mathbf{5 3 . 2 \%}$ & $\mathbf{1}$ \\
Sailing & 340 & $9.8 \%$ & \\
Swimming & 627 & $18.2 \%$ & \\
Skiing/Water-sports & 452 & $13.1 \%$ & \\
Beach Camping & 222 & $6.4 \%$ & \\
Jet Skiing & 39 & $1.1 \%$ & \\
Other & 118 & $3.4 \%$ & \\
\hline
\end{tabular}

Note that $n=3,446$ total survey respondents to Question 16 . 
- Fishing ranked as the leading activity among the $n=1,698$ survey participants that responded to Question 16 and accessed the water from public Boat Ramps; with slightly over 73\% of these respondents acknowledging that they engaged in this activity during a typical trip.

- Cruising and Sightseeing occurred during approximately 26\% of reported trips originating from Boat Ramps.

- Socialization and Swimming rounded out the top-five activities, with a little over $18 \%$ of respondents indicating that these activities occur during a typical trip (Table 16a; Question 16).

Table 16a. Boaters’ Activity Statistics: Boat Ramp Group.

\begin{tabular}{lrcc}
\hline & & & \\
Activity & Count & $\begin{array}{c}\text { Percentage of } \\
\text { Respondents }\end{array}$ & Rank \\
Beach Picnicking & 290 & $17.1 \%$ & \\
Nature Viewing & 583 & $17.0 \%$ & \\
Sightseeing & $\mathbf{4 4 3}$ & $\mathbf{2 6 . 1 \%}$ & $\mathbf{3}$ \\
Cruising & $\mathbf{4 5 3}$ & $\mathbf{2 6 . 6 \%}$ & $\mathbf{2}$ \\
Daytime Anchoring & 218 & $12.8 \%$ & \\
Socializing & $\mathbf{3 1 4}$ & $\mathbf{1 8 . 5 \%}$ & $\mathbf{4}$ \\
Diving & 169 & $9.9 \%$ & \\
Overnight Anchoring & 99 & $5.8 \%$ & \\
Visit Restaurants & 211 & $12.4 \%$ & \\
Fishing & $\mathbf{1 2 4 6}$ & $\mathbf{7 3 . 3 \%}$ & $\mathbf{1}$ \\
Sailing & 43 & $2.5 \%$ & \\
Swimming & $\mathbf{3 0 8}$ & $\mathbf{1 8 . 1 \%}$ & $\mathbf{5}$ \\
Skiing/Water-sports & 229 & $13.4 \%$ & \\
Beach Camping & 155 & $9.1 \%$ & \\
Jet Skiing & 19 & $1.1 \%$ & \\
Other & 55 & $3.2 \%$ & \\
& & & \\
Results based on n = 1,698 respondents using Boat Ramps. &
\end{tabular}


- Cruising was the number-one activity for the $n=464$ survey respondents departing from Marina Wet Slips, with slightly more than $50 \%$ of respondents indicating that they engage in this activity during a typical trip.

- Sailing and Nature Viewing ranked second and third, with $41 \%$ and 34\%, respectively. Socializing and Overnight Anchoring rounded out the top-five responses.

- Note that less than $10 \%$ of respondents who departed from Marina Wet Slips indicated that they engage in jet skiing, water skiing/water-sports, beach camping, and diving during a typical outing.

- Note that only about one-quarter of survey respondents departing from Marina Wet Slips reported that they engage in fishing during a typical trip (Table 16b; Question 16).

Table 16b. Boaters’ Activity Statistics: Marina Wet Slip Group.

\begin{tabular}{lrcr}
\hline & & & \\
Activity & Count & $\begin{array}{c}\text { Percentage of } \\
\text { Respondents }\end{array}$ & Rank \\
Beach Picnicking & 55 & $11.8 \%$ & \\
Nature Viewing & $\mathbf{1 6 9}$ & $\mathbf{3 6 . 4 \%}$ & $\mathbf{3}$ \\
Sightseeing & 126 & $27.1 \%$ & \\
Cruising & $\mathbf{2 3 4}$ & $\mathbf{5 0 . 4 \%}$ & $\mathbf{1}$ \\
Daytime Anchoring & 139 & $29.9 \%$ & \\
Socializing & $\mathbf{1 6 0}$ & $\mathbf{3 4 . 4 \%}$ & $\mathbf{4}$ \\
Diving & 41 & $8.8 \%$ & \\
Overnight Anchoring & $\mathbf{1 5 2}$ & $\mathbf{3 2 . 7 \%}$ & $\mathbf{5}$ \\
Visit Restaurants & 99 & $21.3 \%$ & \\
Fishing & 114 & $24.5 \%$ & \\
Sailing & $\mathbf{1 9 2}$ & $\mathbf{4 1 . 3 \%}$ & $\mathbf{2}$ \\
Swimming & 91 & $19.6 \%$ & \\
Skiing/Water-sports & 13 & $2.8 \%$ & \\
Beach Camping & 13 & $2.8 \%$ & \\
Jet Skiing & 1 & $.2 \%$ & \\
Other & 20 & $4.3 \%$ & \\
& & & \\
Results based on n = 464 respondents departing from Marina Wet Slips. &
\end{tabular}


- Fishing was the top-ranked activity among respondents who accessed waterways from Marina Dry Storage facilities, with slightly over $57 \%$ indicating that they engage in this activity during a typical trip.

- Cruising was the second-most common response for Marina Dry Storage facility users--an activity that occurs on approximately $26 \%$ of typical trips taken by this group of survey respondents. Nature Viewing, Sightseeing, and Socializing were also found to be top-five activities, each accounting for over $18 \%$.

- Less than $10 \%$ of survey respondents departing from Marina Dry Storage facilities identified sailing, skiing/water-sports, beach camping, and overnight anchoring as activities that occur on a typical trip (Table 16c; Question 16).

Table 16c. Boaters’ Activity Statistics: Marina Dry Storage Group.

\begin{tabular}{lrrr}
\hline & & & \\
Activity & Count & $\begin{array}{r}\text { Percentage of } \\
\text { Respondents }\end{array}$ & Rank \\
Beach Picnicking & 13 & $10.1 \%$ & \\
Nature Viewing & $\mathbf{2 6}$ & $\mathbf{2 0 . 1 \%}$ & $\mathbf{3}$ \\
Sightseeing & $\mathbf{2 3}$ & $\mathbf{1 8 . 8 \%}$ & $\mathbf{4}$ \\
Cruising & $\mathbf{3 4}$ & $\mathbf{2 6 . 3 \%}$ & $\mathbf{2}$ \\
Daytime Anchoring & 16 & $12.4 \%$ & \\
Socializing & $\mathbf{2 4}$ & $\mathbf{1 8 . 6 \%}$ & $\mathbf{5}$ \\
Diving & 13 & $10.1 \%$ & \\
Overnight Anchoring & 9 & $6.9 \%$ & \\
Visit Restaurants & 25 & $19.3 \%$ & \\
Fishing & $\mathbf{7 4}$ & $\mathbf{5 7 . 3 \%}$ & $\mathbf{1}$ \\
Sailing & 3 & $2.3 \%$ & \\
Swimming & 13 & $10.1 \%$ & \\
Skiing/Water-sports & 12 & $9.3 \%$ & \\
Beach Camping & 7 & $5.4 \%$ & \\
Jet Skiing & 2 & $1.5 \%$ & \\
Other & 6 & $4.6 \%$ & \\
& & & \\
Results based on n = 129 survey respondents departing from Marina Dry \\
Storage facilities.
\end{tabular}


- Cruising ranked as the top activity of survey respondents departing from Docks, with $42 \%$ acknowledging that this activity takes place during a typical trip.

- Fishing was the second-most common response, reported by 39\% of Dock users.

- Other activities that this user group identified as prominent included nature viewing (32\%), sightseeing (31\%), and socializing (31\%).

- The least-likely activities for respondents departing from private Docks included diving, beach camping, sailing, jet skiing, and skiing/water-sports; each accounting for less than 10\% of the activities identified as occurring during a typical trip (Table 16d; Question 16).

Table 16d. Boaters’ Activity Statistics: Dock Group.

\begin{tabular}{lrcc}
\hline & & & \\
Activity & Count & $\begin{array}{c}\text { Percentage of } \\
\text { Respondents }\end{array}$ & Rank \\
Beach Picnicking & 170 & $17.8 \%$ & \\
Nature Viewing & $\mathbf{3 0 5}$ & $\mathbf{3 2 . 1 \%}$ & $\mathbf{3}$ \\
Sightseeing & $\mathbf{3 0 1}$ & $\mathbf{3 1 . 6 \%}$ & $\mathbf{4}$ \\
Cruising & $\mathbf{3 9 9}$ & $\mathbf{4 2 . 0 \%}$ & $\mathbf{1}$ \\
Daytime Anchoring & 180 & $18.9 \%$ & \\
Socializing & $\mathbf{3 0 0}$ & $\mathbf{3 1 . 5 \%}$ & $\mathbf{5}$ \\
Diving & 70 & $7.3 \%$ & \\
Overnight Anchoring & 100 & $10.5 \%$ & \\
Visit Restaurants & 261 & $27.4 \%$ & \\
Fishing & $\mathbf{3 7 4}$ & $\mathbf{3 9 . 3 \%}$ & $\mathbf{2}$ \\
Sailing & 93 & $9.7 \%$ & \\
Swimming & 199 & $20.9 \%$ & \\
Skiing/Water-sports & 190 & $20.0 \%$ & \\
Beach Camping & 43 & $4.5 \%$ & \\
Jet Skiing & 17 & $1.7 \%$ & \\
Other & 30 & $3.1 \%$ & \\
& & & \\
Results based on n = 950 survey respondents departing from private Docks. &
\end{tabular}




\section{Perceived Congestion}

This section offers a summary of "perceived congestion" statistics based on survey responses to Question 17. Note that congestion was defined in the questionnaire as the presence of "more boaters that you would prefer" in a given area. The results are shown in Table 17 and highlighted in Figure 4.

- Approximately $40 \%$ of the boaters that participated in the survey answered, "Yes" to Question 17, indicating that they had avoided or left congested areas while boating.

- Survey respondents departing from Boat Ramps had the highest percentage of perceived congestion with approximately 50\% indicating that they had left or avoided an area that they perceived as being congested. This value was significantly higher than the average across all waterway access groups.

- Respondents departing from the Shoreline/Causeway also indicated a relatively high percentage of perceived congestion (43\%); although it was a percentage that was not found to be significantly different from the overall average value of $40 \%$ (due to the relatively small sample size).

- Of the primary water access groups, respondents departing from Marina Wet Slips were the least likely to perceive congestion on the waterways, with only $22 \%$ reporting that they had left or avoided an area due to congestion. This percentage was significantly less than the average overall percent of perceived congestion (40\%).

- Respondents who departed from both private Docks and Marina Dry Storage facilities had perceived congestion percentages that were significantly less than the overall average of $40 \%$, with $30 \%$ and $31 \%$, respectively (Table 17; Question 17= Yes). 
Table 17. Proportion of Boaters that Indicated They Had Avoided or Left Their Favorite Spots/Destinations Due to Congestion.

\begin{tabular}{lcccc}
\hline Access Category & $\mathbf{n}$ & $\begin{array}{r}\text { Answered } \\
\text { "Yes” to Q17 }\end{array}$ & Percentage & Rank \\
Boat Ramp & $\mathbf{1 3 3 9}$ & $\mathbf{6 6 7}$ & $\mathbf{4 9 . 8 \% * *}$ & $\mathbf{1}$ \\
Shoreline/Causeway & $30 \dagger$ & 13 & 43.3 & 2 \\
Marina Wet Slip & 328 & 74 & $22.5 \% *$ & 5 \\
Marina Dry Storage & 86 & 27 & $31.4 \% *$ & 3 \\
Dock & 606 & 185 & $30.5 \% *$ & 4 \\
Other (not ranked) & $11 \dagger$ & 3 & $27.2 \%$ & $\mathrm{NA}$ \\
Overall & $\mathrm{n}=2,405$ & 971 & $40.3 \%$ (average) \\
\hline
\end{tabular}

$\dagger$ indicates a relatively small sample size $(\mathrm{n}<40)$.

* Denotes a percentage value that is significantly less than the overall average \% at $95 \%$ Confidence.

** Denotes a percentage value that is significantly greater than the overall average $\%$ at $95 \%$ Confidence.

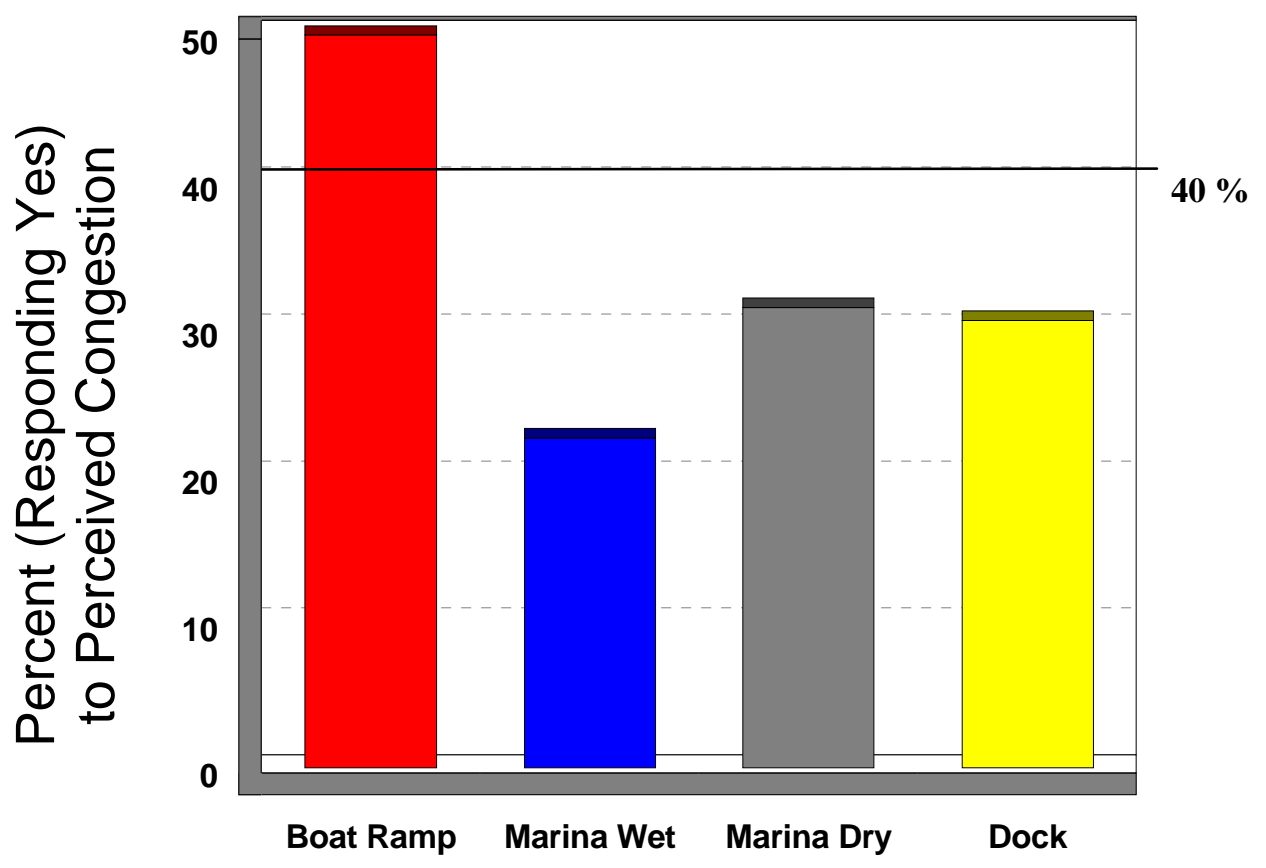

Figure 4. Percentage of Survey Respondents Reporting "Yes” to Perceived Congestion By User Group. 


\section{Chapter 5. Seasonal Boating Characteristics}

\section{Defining the Boating Seasons - A Temporal Analysis}

Monthly trip data were examined to identify the number and the duration of boating seasons, based upon the average number of trips taken by boaters during each month and related statistics. Trip frequency counts - the number of reported boating days - were obtained from responses to Question 13 of the mail survey instrument (see appendix A for the Questionnaire).

For the purposes of this analysis, a boating season is defined as a grouping of "like" consecutive or non-consecutive months based on temporal trends in waterway use and monthly trip frequency counts. The mean (average) of the number of reported trips (per month) are shown in Table 18 and highlighted in Figure 5. Summary statistics are presented for all survey respondents and for each of four distinct waterway access groups - comprised of boaters/respondents accessing the waterways from Boat Ramps, Marina Wet Slips, Marina Dry Storage facilities, and Docks.

Visual inspection of the mean number of trips for all waterway access groups (Figure 5) exposes a pattern that is consistent with defining three boating seasons in the Brevard County study region. Identifiable clusters of "like months," based on similarities in trip frequencies, suggest the presence of a primary "peak" season, an "off-peak" season, and a non-consecutive month "shoulder" season (associated with months that serve to bridge the peak and non-peak seasons).

1. The "peak season" is centered about the month of June (and runs from May through July), where the average reported trip count per respondent/boater is between 3.41 and 3.63 trips per month (values that are significantly greater than the overall average of 2.82 trips per month at the $95 \%$ confidence level);

2. A low use-intensity "off-peak" season that spans from November through February, where the average reported trip count ranges between 2.02 and 2.33 trips per month (values that are significantly less than the overall average of 2.82 trips per month at the $95 \%$ confidence level); and

3. A "shoulder" season that bridges the peak and non-peak seasons - comprised of a twomonth pre-peak cluster (March and April) and a three-month post-peak cluster (which runs from August through October).

Note that the shoulder months are associated with average trip counts that are not significantly different from the overall monthly average value of 2.82 trips per month. The pre-peak shoulder period (March and April) is associated with a gradual rise in average trip counts per month, while the post-peak shoulder period exhibits a marked decline in the average reported trip counts per month. 
The average monthly trip count for the pre-peak shoulder period (March and April) is approximately 3.0 trips per month and the average monthly trip count for the post-peak shoulder period (August through October) is 2.8 trips per month. In both cases, theses multiple month cluster averages are not significantly different from the overall average monthly trip count of 2.82 trips per month at the $95 \%$ confidence level.

Table 18. Mean Number of Reported Trips by Month and User Category.

\section{Mean Number of Reported Trips}

\begin{tabular}{|c|c|c|c|c|c|c|}
\hline Month & All & Ramp & Dock & $\begin{array}{l}\text { Marina } \\
\text { Wet Slip }\end{array}$ & $\begin{array}{c}\text { Marina } \\
\text { Dry Storage }\end{array}$ & $\begin{array}{l}\text { 95\% Confidence } \\
\text { Internal (mean) } \\
\text { All Users } \dagger\end{array}$ \\
\hline January & 2.07 & 2.21 & 1.73 & 2.38 & 1.61 & $(1.95-2.19)$ \\
\hline February & 2.16 & 2.32 & 1.82 & 2.35 & 1.83 & $(2.04-2.28)$ \\
\hline March & 2.79 & 3.03 & 2.37 & 2.90 & 2.45 & $(2.65-2.93)$ \\
\hline April & 3.23 & 3.46 & 2.85 & 3.30 & 2.88 & $(2.82-3.64)$ \\
\hline May* & 3.62 & 3.84 & 3.28 & 3.61 & 3.26 & $(3.46-3.78)$ \\
\hline June* & 3.63 & 3.85 & 3.39 & 3.43 & 3.25 & $(3.47-3.80)$ \\
\hline July* & 3.41 & 3.68 & 3.23 & 2.88 & 2.91 & $(3.25-3.57)$ \\
\hline August & 3.08 & 3.32 & 2.90 & 2.63 & 2.77 & $(2.73-3.43)$ \\
\hline September & 2.88 & 3.09 & 2.63 & 2.68 & 2.55 & $(2.74-3.02)$ \\
\hline October & 2.67 & 2.81 & 2.45 & 2.72 & 2.24 & $(2.52-2.82)$ \\
\hline November & 2.33 & 2.44 & 2.06 & 2.62 & 1.83 & $(2.20-2.46)$ \\
\hline December & 2.02 & 2.11 & 1.73 & 2.42 & 1.56 & $(1.90-2.14)$ \\
\hline $\begin{array}{l}\text { Monthly Avg. } \\
\text { (overall)** } \mathrm{n}=\end{array}$ & $\begin{array}{r}2.82 \\
3,116\end{array}$ & $\begin{array}{r}3.01 \\
1652\end{array}$ & $\begin{array}{r}2.53 \\
900\end{array}$ & $\begin{array}{r}2.82 \\
441\end{array}$ & $\begin{array}{r}2.43 \\
123\end{array}$ & $(2.70-2.94)$ \\
\hline
\end{tabular}

* Peak months, where mean $>2.82$ at $95 \%$ confidence.

** Excludes respondents from Shoreline/Causeway or Other category.

† 95\% Confidence Intervals are shown in parentheses, and are interpreted as follows:

Bold: if confidence interval does not contain the mean of 2.82 trips and mean $>2.82$;

Bold and Italic: if the upper limit of confidence interval $<2.82$ trips. 


\section{Mean Trip Counts by Month}

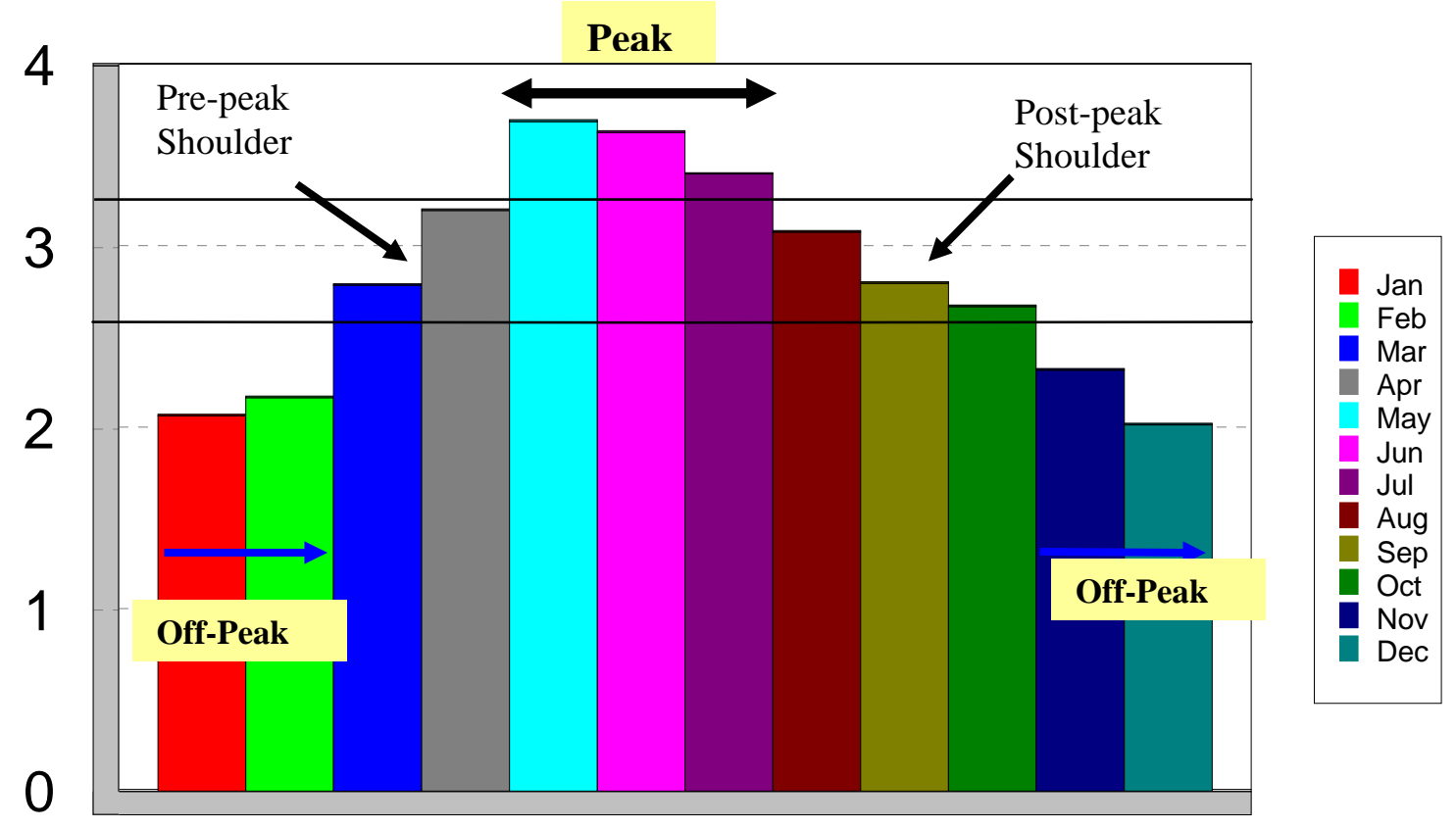

Figure 5. Mean Monthly Trip Counts (All Survey Respondents). 


\section{Sample Size Considerations}

Average monthly trip counts are based on user information obtained from Question 13 of the survey questionnaire, in which survey respondents were asked to report the number of boating trips taken during each month of the year. The observed maximum estimated standard deviation $\mathrm{s}_{\max }$ for monthly trip counts of all respondents was approximately 3.5 trips per month. This value implies that a minimal required sample size ( $\mathrm{n}^{*}$ ) of approximately 310 respondents to be within an acceptable margin of error - plus or minus .5 trips per month - when estimating the mean monthly trip count at the $95 \%$ confidence level. As such, the sample of $n=3,116$ respondents reported trips easily exceeds the number required to meet the specified margin of error when generating estimates for the average number of monthly trips for all respondents.

In the validation of individual sample sizes associated with the various waterway access/user groups, questions do arise over the adequacy of the size of sub-samples. There is statistical evidence, however, to suggest that an adequate sample size for each user group was obtained based on the estimated standard deviations associated with reported monthly trip counts. For example, consider that for a $\mathrm{j}$-th user category $(\mathrm{j}=1, \ldots, 4$ waterway access/user groups) for any given k-th month $(\mathrm{k}=1, \ldots 12)$, the average estimated standard deviation of reported monthly trip counts for a $\mathrm{j}$-th user category and $\mathrm{k}$-th month $\left(\mathrm{s}^{*}{ }_{\mathrm{jk}}\right)$ is approximately 4.0 . This value implies that a minimum sample size of approximately 330 is required for each user group to be within the prescribed margin of error (plus or minus .5 trips). This sub-sample target is somewhat overstated, however, as it does not take into account the "finite" nature of the various boater populations associated with each of the four major water access groups.

The required sample size of 330 trip observations is exceeded for all user categories with the exception of boaters that fall in the Marina Dry Storage (with a sub-sample count of only $\mathrm{n}=245$ ). Adjusting for the finite nature of the boating population within this category, based on a rough estimate of Marina Dry Storage availability within the region and the standard deviation in monthly trip counts, the estimated minimum required sample size is approximately 120 to fall within an acceptable maximum margin of error - plus or minus .5 trips per month at a $90 \%$ confidence level. Hence, the samples size of each waterway access category obtained from the survey questionnaire may be deemed as appropriate for the purposes and objectives of this study and the desired level of precision. 


\section{Validation of Designated Boating Seasons}

The three designated boating seasons described earlier were validated by the results of a cluster analysis. Several hierarchical clustering routines were run using monthly data for the variables listed in Table 19, each yielding consistent results. The clustering routines were constrained to search for an optimal number of clusters $\mathrm{c}^{*}$, based upon an inspection of natural breaks found within the trip data and the patterns associated variables that capture the temporal trend in reported trips. Furthermore, the cluster analysis was run under the imposed minimum of two clusters and a maximum of five clusters in the identification of 'like months.' Hierarchical clustering routines were chosen given that the variables used to describe the trends in Figure 5 were measured at a variety of different scales (i.e., the analysis involved the use of nominal, ordinal, and interval scale data).

Hierarchical clustering methods were used to identify clusters of months that exhibited "similar" characteristics in terms of the average reported trips, the relative position of months with respect to the peak trip months, the monthly moving average in comparison to the overall average, and monthly trip rankings. Similarity, and hence the clustering of "like months," is determined by the shortest statistical "distance" (i.e., the least dissimilarity between cluster values) by which months or clusters of months are linked together in relational or statistical space (measured in Euclidean terms).

In short, individual months and clusters of months were linked in a manner that is efficient in terms of accounting for variation, similarities/dissimilarities, and/or differences in the values of monthly observations for the variables listed in Table 19.

Table 19. A Listing and Description of Variables Used in the Hierarchical Cluster Analysis.

Cluster/Label Variable: $\mathbf{M O N T H}$ (month of the year): January - December

Variables used to cluster MONTH* $^{*}$

$\begin{array}{ll}\text { Variable } & \text { Description } \\ \text { ANRT } & \text { Average Number of Reported Trips (per month) } \\ \text { DISTP } & \text { Distance from Peak center-- absolute number of months } \\ \text { Rank } & \text { Rank of ANRT (in descending order } \rightarrow \text { 1=high; 12=low) } \\ \text { MA3_Rank } & \text { Moving Average of Rank (3 } 3^{\text {rd }} \text {, centered) } \\ \text { INC_ANRT } & \text { Increase in ANRT (over previous month) } \\ \text { AATM } & \text { Above-Average Trip Month (1=yes; } 0=\text { no) }\end{array}$

* Note that the variables listed above are measured at a variety of scales, including the nominal, ordinal, and interval scale; requiring clustering methods that allow for "mixed" data types. 


\section{Dendrograms}

Cluster routines are typically accompanied by a "dendrogram" - a graphical device that displays the distance (or dissimilarity) between clusters, and the distance at which individual objects or clusters are joined. This device offers a way in which to map the distances at which various clusters join. It also allows for the identification of break points that exist between various clusters or cluster groupings, and is an historical account of the clustering process as individual elements/months are linked together. Dissimilarity distances and break points (highlighting large gaps between clusters) are the basis by which an optimal number of clusters can be determined. In short, the dendrogram is the graphical counterpart to the summary statistics generated from a given cluster routine, and includes information on dissimilarity distances and the values at which clusters form and link together.

Summary statistics for the cluster analysis on reported monthly trips and the designation of boating seasons are provided in Table 20 for a selected number of hierarchical clustering methods. In all the hierarchical routines employed, the clustering algorithms produced identical groupings or clusters of months. Note also that the selected cluster routines each produced cophenetic correlation coefficients that ranged between .64 and .73 - indicating that the identified cluster groupings are strong and efficient in terms of representing the similarities/dissimilarities that exist in the values of the variables associated with the different months of the year. The results suggest that the non-consecutive months of August, September, October, March, and April form a distinct cluster whose members are similar in terms of trip statistics, yet dissimilar and differentiable from months not contained within this cluster. In other words, these five shoulder months are statistically dissimilar to the months forming the "peak" season cluster and those forming the "off-peak" season cluster. 
Table 20. Results of Cluster Analysis in the Designation of Boating Seasons.

Distance Values for Clusters and Cluster Links $\dagger$

\begin{tabular}{|c|c|c|c|c|}
\hline $\begin{array}{r}\text { Routine } \rightarrow \\
\text { Distance type } \rightarrow\end{array}$ & $\begin{array}{l}\text { Simple Avg. } \\
\text { Weighted } \\
\text { (Euclidean) }\end{array}$ & $\begin{array}{l}\text { Avg. Absolute } \\
\text { Deviation Centroid } \\
\text { (Euclidean) }\end{array}$ & $\begin{array}{l}\text { Complete } \\
\text { Un-Weighted } \\
\text { (Euclidean) }\end{array}$ & $\begin{array}{l}\text { Median } \\
\text { Weighted } \\
\text { (Euclidean) }\end{array}$ \\
\hline
\end{tabular}

Cluster(s) identified: 1,2 and 3

Peak

1 June

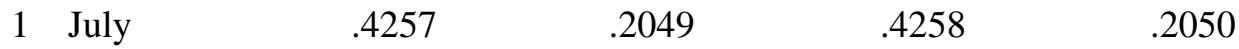

1 May (May links to July to start cluster - very similar)

\section{Off-Peak}

2 November

$\begin{array}{llllll}2 & \text { February } & .5430 & .2470 & .4669 & .2470\end{array}$

2 December

2 January (January links to December to start cluster - very similar)

\section{Shoulder (Non-consecutive)}

3 August (August links to April to start cluster - very similar)

3 April

$\begin{array}{llllll}3 & \text { October } & .8202 & .5583 & .7538 & .6627\end{array}$

3 September

3 March (March links to September to start cluster - very similar)

Cluster links:

$\begin{array}{lcccc}2-3 & 1.3795 & 1.7439 & 1.3393 & 1.8503 \\ 1-2-3 & 2.0386 & 3.5906 & 1.9202 & 4.0566 \\ -\begin{array}{l}\text { Cophenetic } \\ \text { Correlation }\end{array} & .7364 & .6418 & .7368 & .6416 \\ \begin{array}{l}\text { Suggested \# } \\ \text { of clusters }\end{array} & 3 & 3 & 3 & 3\end{array}$

Note: Cluster routines were run using NCSS 2000

$\dagger$ Note: Similar cluster designations were also produced using a Manhattan metric distance. 
A more detailed account of the step-by-step clustering process is provided by the dendrograms shown in Figures 6a and 6b. The vertical axis of the dendrogram illustrates the months or clusters of months as they link together. The horizontal axis yields a measure of dissimilarity and the distance at which months or clusters fuse together. The observed gaps between clusters reveal three distinct break points based on observed maximum distances in dissimilarity between months or clusters. The horizontal axis provides a platform for viewing the positioning of each object as it is clustered and, in this case, how months and clusters of months are linked and arranged in relational space at any given dissimilarity value.

The dendrogram is useful in visualizing the distance at which any two months and/or clusters are fused together and the degree of dissimilarity between those months or clusters. The less the dissimilarity, the faster months or clusters link together. For example, the dendrogram in Figure 6a suggests that the months of May, June, and July are very dissimilar to the months of November, December, January, and February, as these two clusters are the last to be joined together at a distance of approximately 3.59. The month of March is more like the month of September (joined at a distance of .037) than it is like the month of February (which is joined with March at a distance of 1.743). The month of March is more like the months of September and October than it is May, as it forms a cluster with September and October at a distance of .176, but does not link to May until the clusters link at a distance of 3.59 (the distance in which all months and clusters fuse together). Note also there is a large natural break (or gap) between the cluster of "off-peak" months (which contains November) and the cluster of "shoulder" months (which contains the month of March). Months of the year that are most alike (or least dissimilar) in terms of the number of trips form the "early clusters" located at the far-right side of the dendrogram. Note that these clusters are at distances that are relatively close to zero. For example, consider the pairings of August and April at a distance of .040) or that of July and May (at a distance of .101). In short, dissimilarity between any two months or clusters increases as distance between those months or clusters increases, and as one moves left across the horizontal axis. Note that the dendrogram in Figure 6b is similar in structure to that of the one shown in Figure 6a. In fact, the dendrograms for all selected hierarchical cluster routines shown in Table 20 are very similar to one another. This consistency suggests that the three designated cluster groups/boating seasons are efficient ways in which to group months based on the reported trip data. The results of the cluster analysis also suggest that the optimal number of clusters is 3 , with groupings that match those identified by visual inspection of Figure 5, and the summary statistics in Table 18. The cluster analysis provides statistical validation for the designated groupings of months that define each of the three designated boating seasons - peak season, off-peak season, and a nonconsecutive shoulder season that bridges the peak and non-peak seasons. It is interesting to note that the clusters do not conform to conventional seasonal classifications of winter, spring, summer, and fall. This statistical finding suggests that trip propensity in any given month may be affected by numerous factors including physical conditions (e.g., weather patterns), boater characteristics, and behavioral factors -boaters' perceptions and expectations regarding conditions associated with individual months or time periods, the on-water recreational boating experience as defined by user-related traffic patterns (spatially and temporally), general use patterns by departure category and season, perceptions of congestion and "hot spots," and accessibility to favorite destinations. The results presented in this section form the foundation for the subsequent seasonal analyses in which trip patterns and activities by boating seasons and user groups will be analyzed. 


\section{Dendrogram}

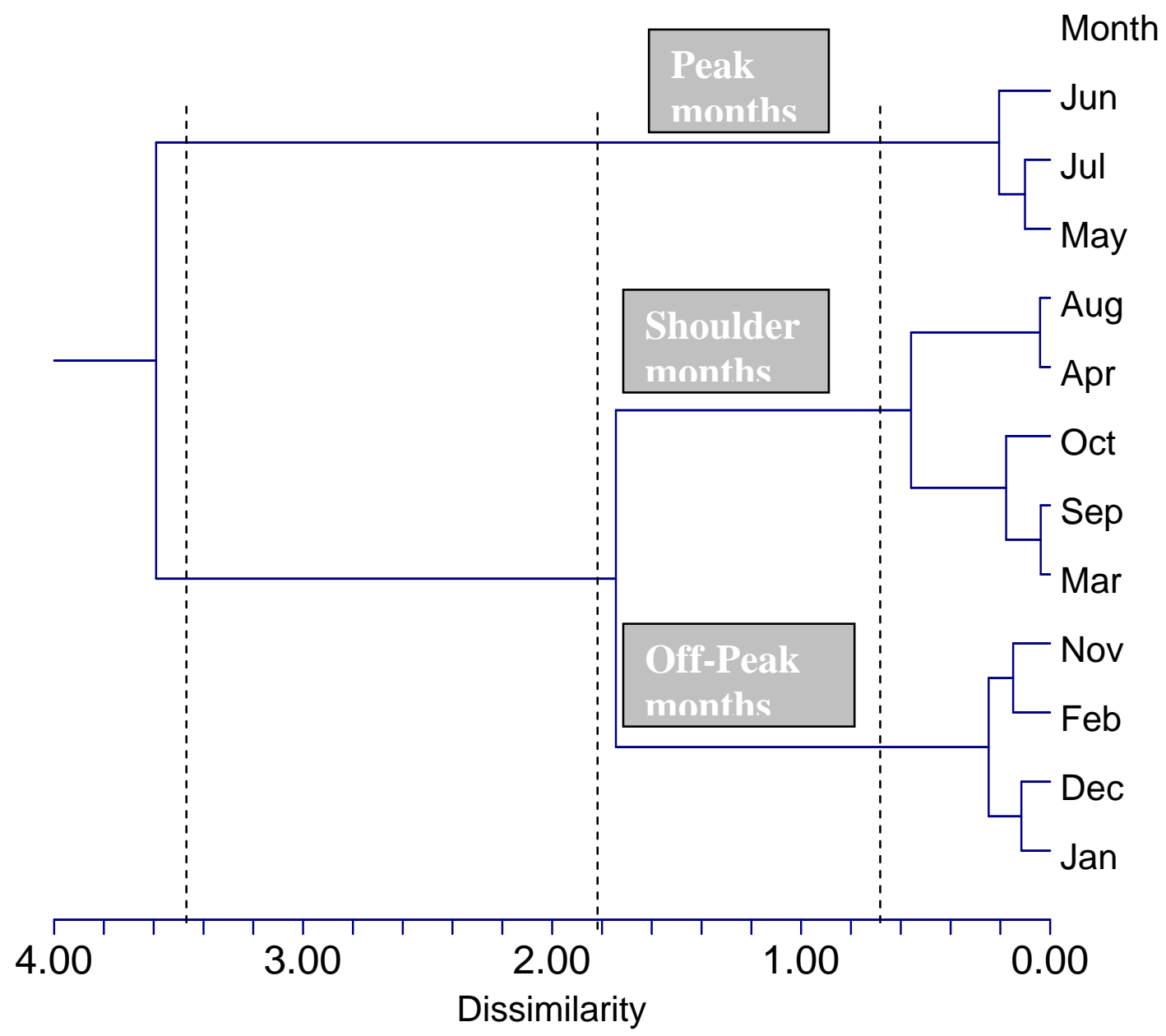

Figure 6a. Dendrogram Showing Clusters of Months Based on the Mean Number of Reported Monthly Trips and Related Variables; Using a Multivariate Hierarchical Cluster Analysis.

(Method: Average Absolute Deviation w/Euclidean Distance). 


\section{Dendrogram}

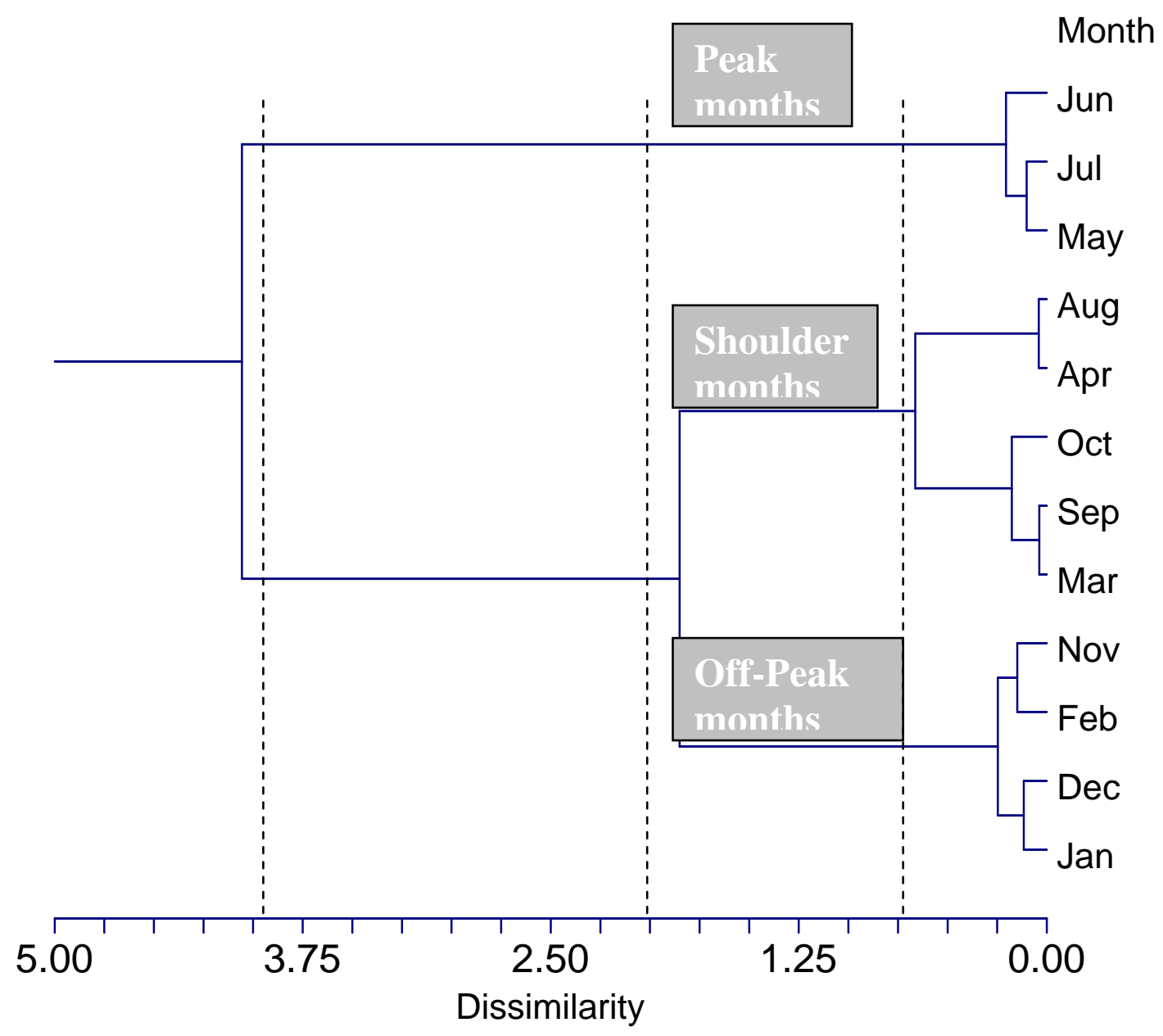

Figure 6b. Dendrogram Showing Clusters of Months Based on the Mean Number of Reported Monthly Trips and Related Variables; Using a Multivariate Hierarchical Cluster Analysis.

(Method: Median Weighted Pair-Group Centroid w/Euclidean Distance). 


\section{Seasonal Survey Participation}

This section examines seasonal boating trip characteristics using the information from the "last two" trips taken by survey recipients. The analysis focuses on seasonal variations in the following trip-related attributes: AM and PM departure time, trip duration (day trips vs. overnight trips), weekend vs. weekday trip proportions, and reported trip activities.

Questionnaire information on various trip characteristics was evaluated seasonally and by user group. The number of reported trips that took place during a given boating season is shown in Table 21. A total of $n=6,332$ seasonal boating trips were reported by respondents to the survey questionnaire. As expected, the largest percentage of reported trips was associated with the peak season (roughly 44\% of all trips). Despite the orientation of the sample toward peak season trips, adequate sample sizes were obtained for the off-peak and shoulder seasons, as well as the majority of season/user-group combinations.

Table 21. Seasonal Survey Participation Among User Groups (Reported Trips).

\begin{tabular}{|c|c|c|c|c|c|c|}
\hline Season & All Users & Ramp & Dock & $\begin{array}{c}\text { Marina } \\
\text { Wet }\end{array}$ & $\begin{array}{c}\text { Marina } \\
\text { Dry }\end{array}$ & Other \\
\hline $\begin{array}{l}\text { Off- } \\
\text { Peak }\end{array}$ & $\begin{array}{r}1,388 \\
21.9 \%\end{array}$ & $\begin{array}{r}690 \\
(49.7 \%) \\
(21.0 \%)\end{array}$ & $\begin{array}{r}414 \\
(29.8 \%) \\
(22.8 \%)\end{array}$ & $\begin{array}{r}200 \\
(14.4 \%) \\
(23.3 \%)\end{array}$ & $\begin{array}{r}53 \\
(3.8 \%) \\
(\mathbf{2 1 . 6 )}\end{array}$ & $\begin{array}{r}31 \\
(<3 \%) \\
(25.2 \%)\end{array}$ \\
\hline Peak & $\begin{array}{r}2,769 \\
43.7 \%\end{array}$ & $\begin{array}{r}1,497 \\
(54.1 \%) \\
(\mathbf{4 5 . 4 \% )}\end{array}$ & $\begin{array}{r}743 \\
(26.8 \%) \\
(\mathbf{4 1 . 2 \% )}\end{array}$ & $\begin{array}{r}361 \\
(13.0 \%) \\
(\mathbf{4 2 . 0 \% )}\end{array}$ & $\begin{array}{r}114 \\
(4.1 \%) \\
(\mathbf{4 6 . 5 \% )}\end{array}$ & $\begin{array}{r}54 \\
(2 \%) \\
(43.9 \%)\end{array}$ \\
\hline Shoulder & $\begin{array}{r}2,175 \\
34.3 \%\end{array}$ & $\begin{array}{r}1,109 \\
(50.1 \%) \\
(33.6 \%)\end{array}$ & $\begin{array}{r}652 \\
(29.9 \%) \\
(\mathbf{3 6 . 0 \% )}\end{array}$ & $\begin{array}{r}298 \\
(13.7 \%) \\
(34.7 \%)\end{array}$ & $\begin{array}{r}78 \\
(3.6 \%) \\
(31.8 \%)\end{array}$ & $\begin{array}{r}38 \\
(<3 \%) \\
(30.9 \%)\end{array}$ \\
\hline \multirow[t]{2}{*}{ All } & 6,332 & 3,296 & 1,809 & 859 & 245 & 123 \\
\hline & $\begin{array}{l}\text { Percentage } \\
\text { of all users* }\end{array}$ & $52.0 \%$ & $28.6 \%$ & $13.6 \%$ & $3.9 \%$ & $1.9 \%$ \\
\hline
\end{tabular}

* Survey respondents classified as “Other” - boaters launching from Beach, Shoreline, or Causeway.

Percentages shown in parentheses -- (\%) and (\%) -- are defined as follows: \% of user group in non-bold type; with \% of season total shown in bold type. 


\section{Seasonal Analysis of Number of Trips Taken}

For the seasonal analyses, only the four major waterway access "user groups" are analyzed; specifically, boaters that depart from Ramps, Docks, Marina Wet Slips, and Marina Dry Storage facilities.

Table 22 highlights various summary statistics as they pertain to average and median trips taken during in each of the three designated boating seasons identified by the cluster analysis (based on responses to Question 13 of the survey questionnaire).

Note that each of the three designated boating seasons contained a different number of months: (a) an off-peak season of 4 winter months; (b) a peak season of 3 high-use months; and (c) a shoulder season comprised of 5 non-consecutive months. As a result, trip data was standardized to reflect the mean and median number of reported trips that occurred during a "typical" month within each of the three boating seasons. In other words, the summary statistics on seasonal trips in forthcoming sections will be shown on a season-specific "per month" basis.

It is important to note that all seasonal distributions of reported trips were positively skewed and found to be significantly different from a "normal distribution" at the 95\% confidence level. The implication here is that mean monthly trip counts by season may be somewhat overstated due to the presence of "outliers" (large observations that tend to inflate the averages).

The summary statistics in Table 22 reveal that the mean and median number of trips per month varies substantially across seasons, with the shoulder season being the one that is more like the overall mean and median trip count per month for all seasons. 
Table 22. Seasonal Breakdown of the Mean and Median Number of Trips per Month by Waterway Access/User Group.

\begin{tabular}{|c|c|c|c|c|c|}
\hline Season & $\begin{array}{c}\text { All } \\
\text { Respondents }\end{array}$ & Ramp & Dock & Marina Wet & Marina Dry \\
\hline Off-Peak & $\begin{array}{r}2.06 \\
1\end{array}$ & $\begin{array}{l}2.28 \\
1.25\end{array}$ & $\begin{array}{r}1.83 \\
.5^{* *}\end{array}$ & $\begin{array}{r}2.45 \\
1\end{array}$ & $\begin{array}{l}1.71 \\
.5^{* *}\end{array}$ \\
\hline Shoulder & $\begin{array}{r}2.80 \\
\underline{1.8}\end{array}$ & $\begin{array}{l}3.15 \\
2.2^{*} \\
\end{array}$ & $\begin{array}{r}2.64 \\
1.2^{* *} \\
\end{array}$ & $\begin{array}{r}2.84 \\
\underline{1.6} \\
\end{array}$ & $\begin{array}{r}2.58 \\
1.4^{* *} \\
\end{array}$ \\
\hline Peak & $\begin{array}{r}3.39 \\
2\end{array}$ & $\begin{array}{l}3.78 \\
2.6^{*}\end{array}$ & $\begin{array}{r}3.30 \\
1.6\end{array}$ & $\begin{array}{r}3.32 \\
\underline{1.6} \\
\end{array}$ & $\begin{array}{r}3.13 \\
1.6 \\
\end{array}$ \\
\hline (Overall) & & & & & \\
\hline Mean & 2.70 & 3.02 & 2.53 & 2.84 & 2.43 \\
\hline Median & 1.6 & $2^{*}$ & $1.25^{* *}$ & 1.6 & 1.4 \\
\hline $\begin{array}{l}\text { Sub- } \\
\text { sample } \\
\text { size (n) }\end{array}$ & 3,114 & 1,652 & 900 & 439 & 123 \\
\hline & $\%$ of $n$ & $53.1 \%$ & $28.9 \%$ & $14.1 \%$ & $3.9 \%$ \\
\hline
\end{tabular}

Note: Mean number of trips per month shown in bold type and median in non-bold type.

Note: Underlined median values are found to be not significantly different from the overall median for all users during the season or grouping in question (based on non-parametric test results carried out at the $95 \%$ confidence level).

* Median value is significantly greater than the Seasonal median for all respondents

** Median value is significantly less than the Seasonal median for all respondents 
Figure 7a illustrates the average number of trips per season for all waterway access groups, where the overall observed mean is equal to 2.7 trips per season. The distributions of reported trips counts per month (by season) are illustrated in Figure 7b. These distributions are severely and positively skewed, with a large number of outlying values (as highlighted by the red dots on the vertical box plots). All seasonal trip distributions were found to be "non-normal" at the 95\% confidence level, a feature that negated the possibility of performing a standard Analysis of Variance (ANOVA) test. Subsequently, a Kruskal-Wallis (KW) ANOVA and a Rank Sum Test were used to evaluate the hypothesis of "equality of medians" to compare the median trip counts per month across each of the three designated boating seasons. The test results for the seasonal comparisons are shown in Table 23.

The KW-ANOVA procedure led to rejecting the null hypothesis of equality of medians at the $95 \%$ confidence level. Furthermore, it is shown that the median number of trips taken during the peak boating season is significantly greater than the overall seasonal median of 1.6 trips at $95 \%$ confidence. Also, the median number of trips taken during the off-peak season is significantly less than the overall seasonal median of 1.6 trips at the $95 \%$ confidence level.

It is important to note that while the differences in mean and median trip values may seem small, the implications of these small (yet statistically meaningful) differences are immense when extrapolated across time and the regional boating population at large. We should not forget that the statistical results are standardized on a "per-month basis," as well as couched in terms of a typical respondent/boater. Consider that even a small difference of say +1.2 trips per month (between seasons) may seem inconsequential on the surface, yet when this value is multiplied by thousands of boaters in the area it translates into a difference of great magnitude when the total trip counts of all boaters are tabulated. 


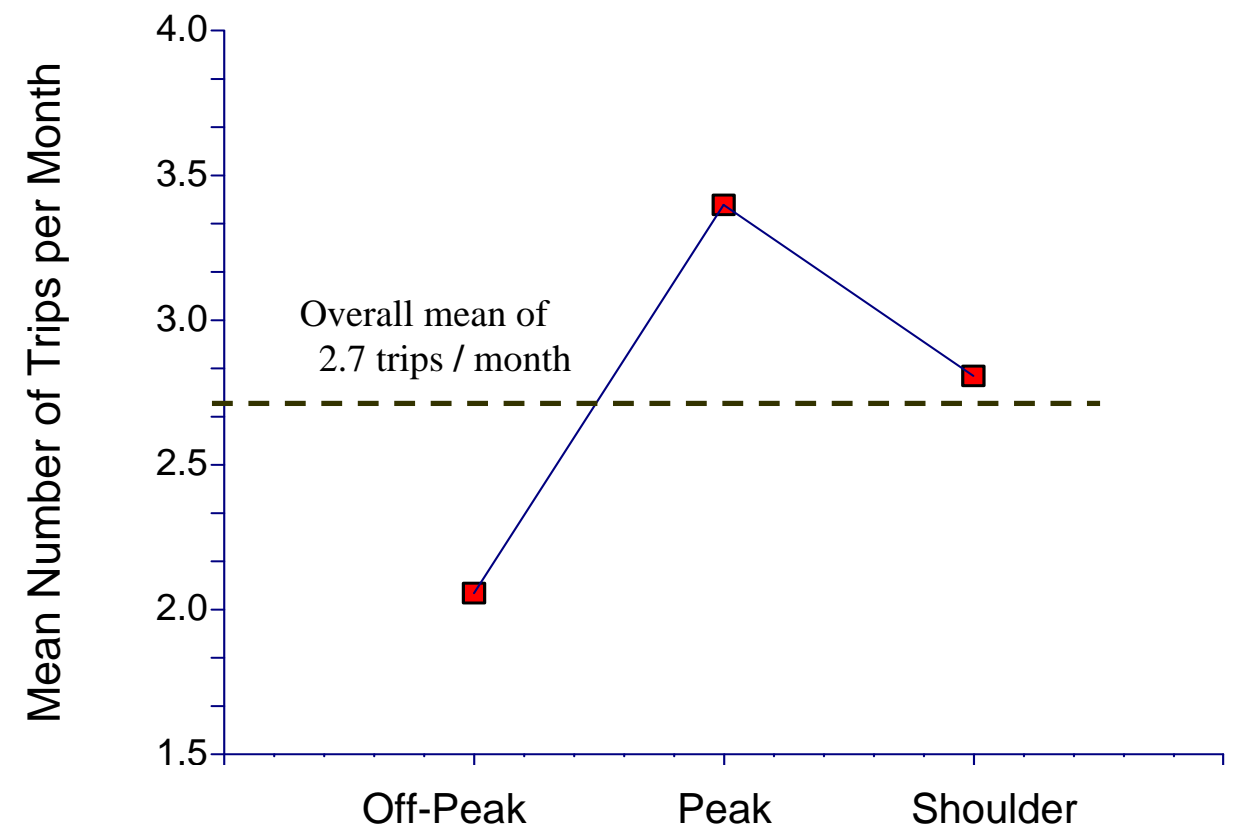

Figure 7a. Mean Trip Counts per Month by Season.

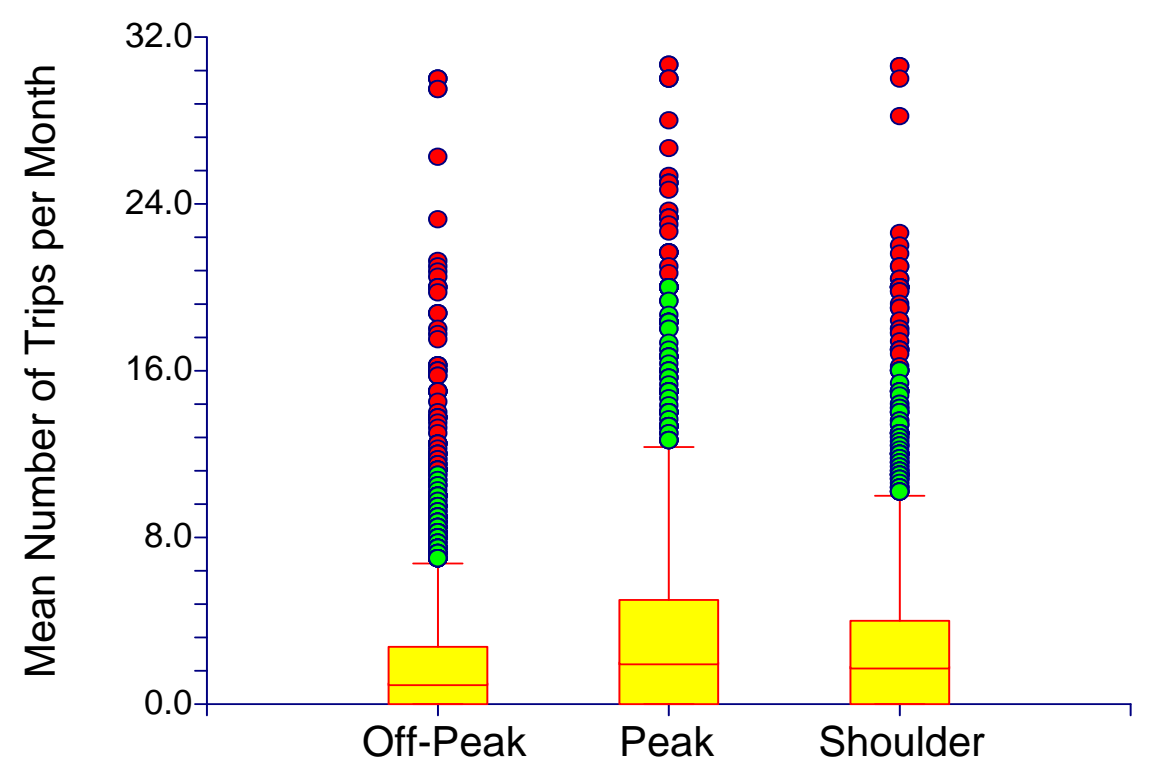

Figure 7b. Monthly Trip-Count Distributions by Season. 
Table 23. Kruskal-Wallis ANOVA and Mean Rank Sum Test Results.

\section{Kruskal-Wallis One-Way ANOVA on Ranks}

\section{Hypotheses}

Ho: All medians are equal (across the designated boating seasons)

Ha: At least two medians are different (across the designated boating seasons)

\section{Test Results}

\begin{tabular}{|c|c|c|c|c|c|}
\hline \multirow{4}{*}{\multicolumn{2}{|c|}{$\begin{array}{l}\text { Method: Rank Sum Test } \\
\text { Not Corrected for Ties } \\
\text { Corrected for Ties } \\
\text { Number Sets of Ties }\end{array}$}} & \multirow{4}{*}{$\begin{array}{l}\text { DF } \\
2 \\
2 \\
147\end{array}$} & \multirow{4}{*}{$\begin{array}{l}\text { Chi-Square } \\
\text { (H) } \\
472.5865 \\
482.3149\end{array}$} & \multirow{4}{*}{$\begin{array}{l}\text { Probability } \\
\text { Level } \\
0.000000 \\
0.000000\end{array}$} & \multirow{4}{*}{$\begin{array}{l}\text { Decision } \\
(\mathbf{0 . 0 5 )} \\
\text { Reject Ho } \\
\text { Reject Ho }\end{array}$} \\
\hline & & & & & \\
\hline & & & & & \\
\hline & & & & & \\
\hline Group & Count & $\begin{array}{l}\text { Sum of } \\
\text { Ranks }\end{array}$ & $\begin{array}{l}\text { Mean } \\
\text { Rank }\end{array}$ & Z-Value & Median \\
\hline Off-Peak & 698 & 799487.00 & 1145.40 & $-13.7250 * *$ & 0.00 \\
\hline Shoulder & 1060 & 1406545.00 & 1326.93 & $-10.2443^{* *}$ & 0.9166 \\
\hline Peak & 1354 & 2637796.00 & 1948.15 & $21.3403 * * *$ & 2.75 \\
\hline
\end{tabular}

Note: Overall median value for a typical boating season $=1.6$ trips per season.

** Significantly less than the overall median of 1.6 at $95 \%$ confidence $* * *$ Significantly greater than the overall median of 1.6 at $95 \%$ confidence 
While the trends in the "mean" or average number of reported trips per month are similar across user groups and seasons (see Table 23 and Figure 11), the mean might not be the best indicator of the average trip generation that occurs within each user group.

In fact, there is a fair amount of variability in the reported trips per month within any given user group and season, as well as outlying observations which tend to skew or overstate the average number of trips taken (as implied by Figure 7b). To sidestep this problem, a comparison was made of the median number of trips taken per month (see Table 23 and Figure 8). Note that Figures 8 and 9 illustrate very different patterns, given that the median values illustrated in Figure 9 are less sensitive to outliers or extreme values; and thus, provide a reasonabe way to represent the typical number of trips that will occur in any given month of a particular season by a given user group.

Analysis of the median trips per month by user group and season, as shown in Figure 9, reveal three distinct seasonal use patterns:

(1) Respondent departing from Boat Ramps tend to report a significantly greater number of trips per month during the peak and shoulder season than respondents associated with other user groups (roughly anywhere from .6 to 1 additional trip per month).

Note again, that while such a small difference in the number of additional trips may seem somewhat inconsequential, the implications are enormous for the total number of trips that this group generates within this study region given that (a) ramp users are a relatively more active boating group in comparison to other user groups when one considers trip frequency and trip duration, (b) they tend spend relatively more time on the water during day trips, and (c) their overall numbers are large in terms of their contribution to the regional boating population.

(2) Dock and Marina Dry Storage facility users tend to report a relatively low number of trips throughout the boating season, with Dock users taking fewer trips per month during the shoulder season in comparison to boaters departing from other launch origins.

(3) Marina Wet Slip users tend to take a median number of trips per month that is not significantly different from the overall season median across all designated boating seasons (something that is confirmed by the results shown in Table 23). 


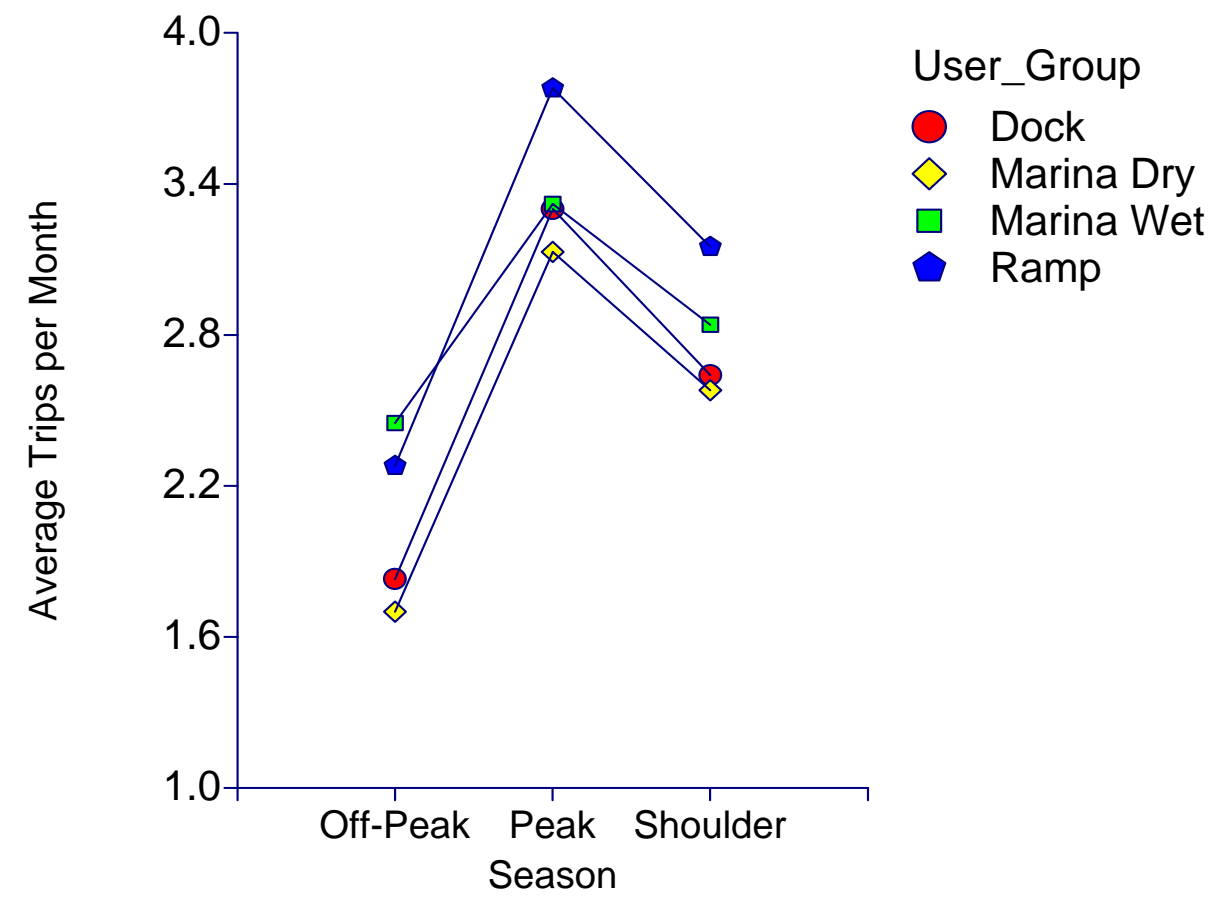

Figure 8. Mean Trip Counts by Season and User Group.

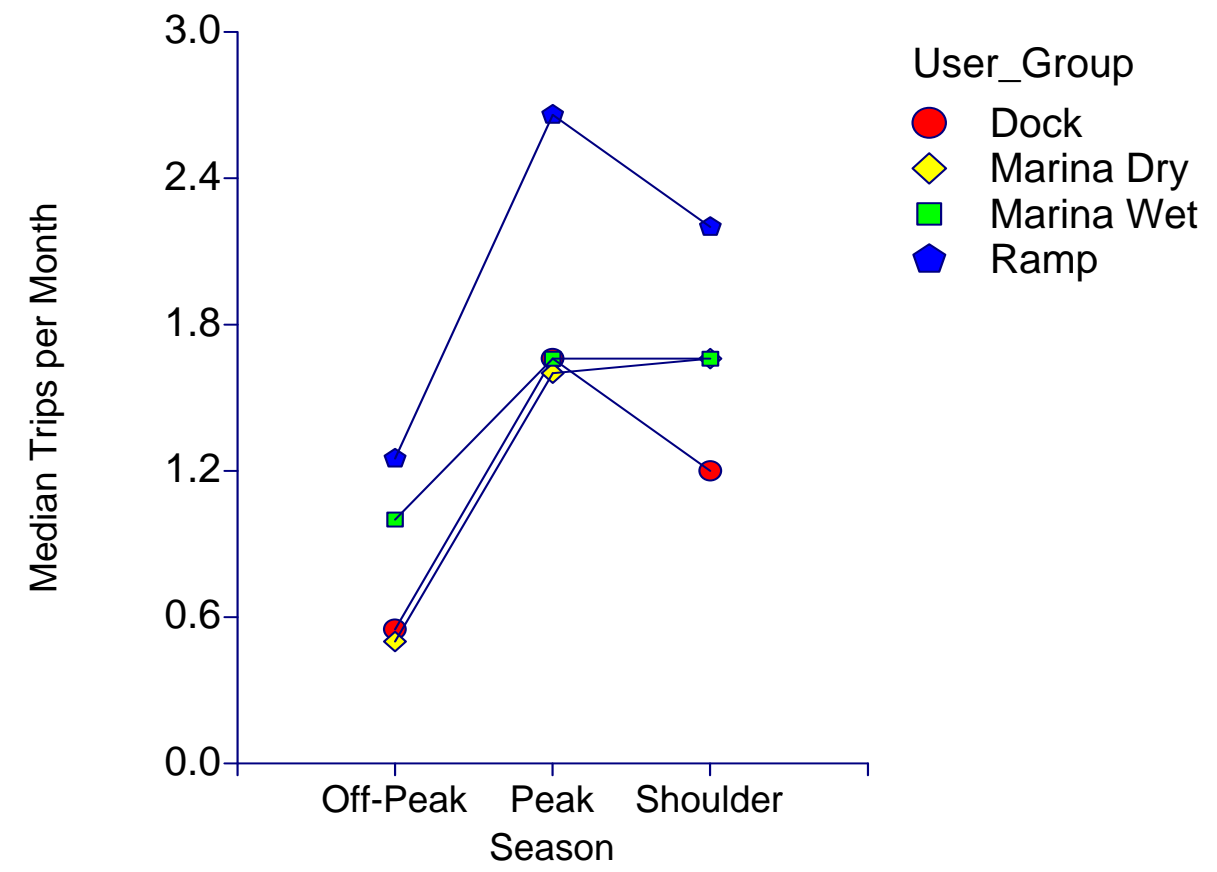

Figure 9. Median Trip Counts by Season and User Group. 


\section{Seasonal Analysis of Trip Departure-Times}

\section{AM Departure Time}

Summary statistics for reported AM departure times by season and user group are presented in Table $24^{1}$. The results are based on information obtained from the $n=5,020$ total survey respondents to Question 2 of the survey questionnaire.

The mean overall AM departure time of trips reported by all survey respondents was approximately 7:52AM. The average peak-season departure time of approximately 7:40AM was found to be significantly earlier than the average departure times during off-peak or shoulder seasons (7:58AM and 8:01AM, respectively), as well as significantly earlier than the overall average of 7:52AM.

Relative frequency histograms highlighting the distributions of reported AM departure times by season are shown in Figures 10a through 10c. Class intervals are broken down by the half-hour to help differentiate the nuances that occur in the AM launch times by season.

The histograms (Figures 10a through 10c) and summary statistics (Table 24) reveal several interesting features that are worthy of enumeration and discussion.

(1) The distribution of AM departure times for each of the three designated boating seasons tends to be "unimodal" (having one prominent peak);

(2) The peak AM departure time tends to occur between 7:00 and 7:30AM during the offpeak and shoulder seasons, and between 6:00 and 6:30AM during the peak season (implying that there is a tendency for boaters to begin their on-water trips earlier during the peak boating season);

(3) There is a "staggering" of non-peak departure times that occur shortly after the hour--with spikes that occur consistently (and shortly) after 8:00AM, 9:00AM, 10:00AM, and 11:00AM in each of the three histograms;

(4) Boaters launching from Ramps begin their trips earlier than other user groups (departing at approximately 7:21AM on average, and 7:12AM during the peak boating season); and

(5) Boaters accessing the waterways from Marina Wet Slips or Docks reported AM departure times that were significantly later than other user groups during all of the designated seasons; with average AM departure times of 8:54AM and 8:31AM, respectively.

\footnotetext{
${ }^{1}$ Note that departure times difference between the peak boating season and shoulder and non-peak seasons may be somewhat understated due to complications the arise with the conversion to Daylight Savings Time.
} 
Table 24. Mean AM Departure Time by Season and User Group.

\begin{tabular}{|c|c|c|c|c|c|}
\hline Season & All Users & Ramp & Dock & $\begin{array}{l}\text { Marina } \\
\text { Wet }\end{array}$ & $\begin{array}{l}\text { Marina } \\
\text { Dry }\end{array}$ \\
\hline Off-Peak & $\begin{array}{r}7: 58 A M \\
(1,072)\end{array}$ & $\begin{array}{l}7: 28 \dagger \\
(614)\end{array}$ & $\begin{array}{c}\text { 9:02**†† } \\
(261)\end{array}$ & $\begin{array}{l}9: 06 \dagger \dagger \\
(134)\end{array}$ & $\begin{array}{c}8: 34 \dagger \dagger * * \\
(40)\end{array}$ \\
\hline Peak & $\begin{array}{c}7: 40 A M^{*} \\
(2,279)\end{array}$ & $\begin{array}{c}7: 12 \dagger \\
(1,355)\end{array}$ & $\begin{array}{c}8: 22 \dagger \dagger \\
(515)\end{array}$ & $\begin{array}{c}\text { 8:42†† } \\
(332)\end{array}$ & $\begin{array}{l}7: 43 \\
(108)\end{array}$ \\
\hline Shoulder & $\begin{array}{c}\text { 8:01AM } \\
(1,669)\end{array}$ & $\begin{array}{l}7: 30 \dagger \\
(955)\end{array}$ & $\begin{array}{c}\text { 8:36*†† } \\
(411)\end{array}$ & $\begin{array}{c}\mathbf{8 : 4 8} * \dagger \dagger \\
(79)\end{array}$ & $\begin{array}{l}8: 03 \\
(67)\end{array}$ \\
\hline $\begin{array}{c}\text { All } \\
\text { Seasons }\end{array}$ & $\begin{array}{c}7: 52 A M \\
(\mathrm{n}=5,020)\end{array}$ & $\begin{array}{c}7: 21 A M+ \\
(2,924)\end{array}$ & $\begin{array}{c}\text { 8:31AM } \dagger \dagger \\
(1,187)\end{array}$ & $\begin{array}{c}8: 54 A M+\dagger \\
(545)\end{array}$ & $\begin{array}{c}7: 59 A M \\
(215)\end{array}$ \\
\hline
\end{tabular}

Note: Mean departure time shown in boldface type; Sample sizes are shown in parentheses.

Note: All Users - represents all departure categories including Shoreline/Causeway and Other.

* Significantly less (earlier) than values observed from the same user group or the overall mean during other seasons at the $95 \%$ confidence;

** Significantly greater (later) than values observed for the same user group or the overall mean value during the other season at the $95 \%$ confidence level;

† Significantly less (earlier) than values observed for other user groups or the overall mean value during the same season at $95 \%$ confidence; and

$\dagger †$ Significantly greater (later) than values observed for other user groups or the overall mean value during the same season at $95 \%$ confidence. 


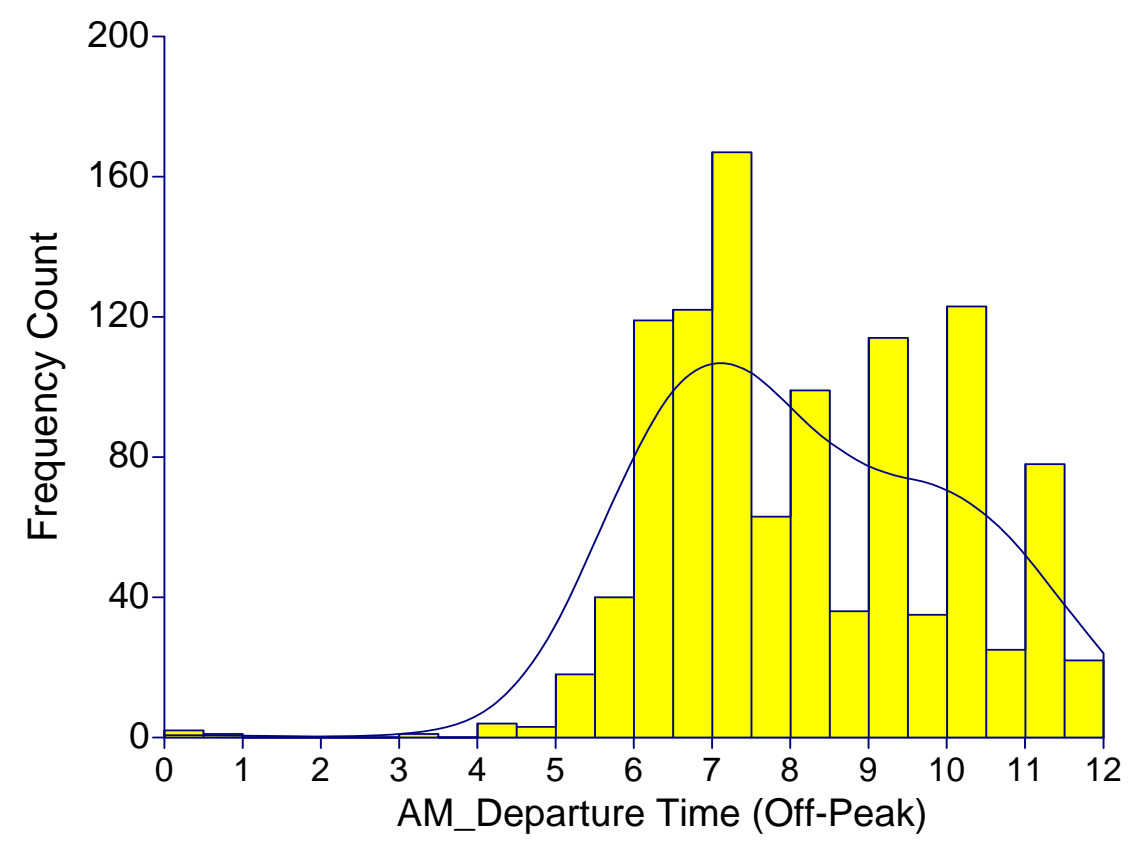

AM Departure Time ( $n=1,072$ respondents)

Note: $\mathrm{X}$-Axis (0.0 = midnight; $12.0=$ noon $)$

Figure 10a. Histogram of AM Departure Time - Non-Peak Season.

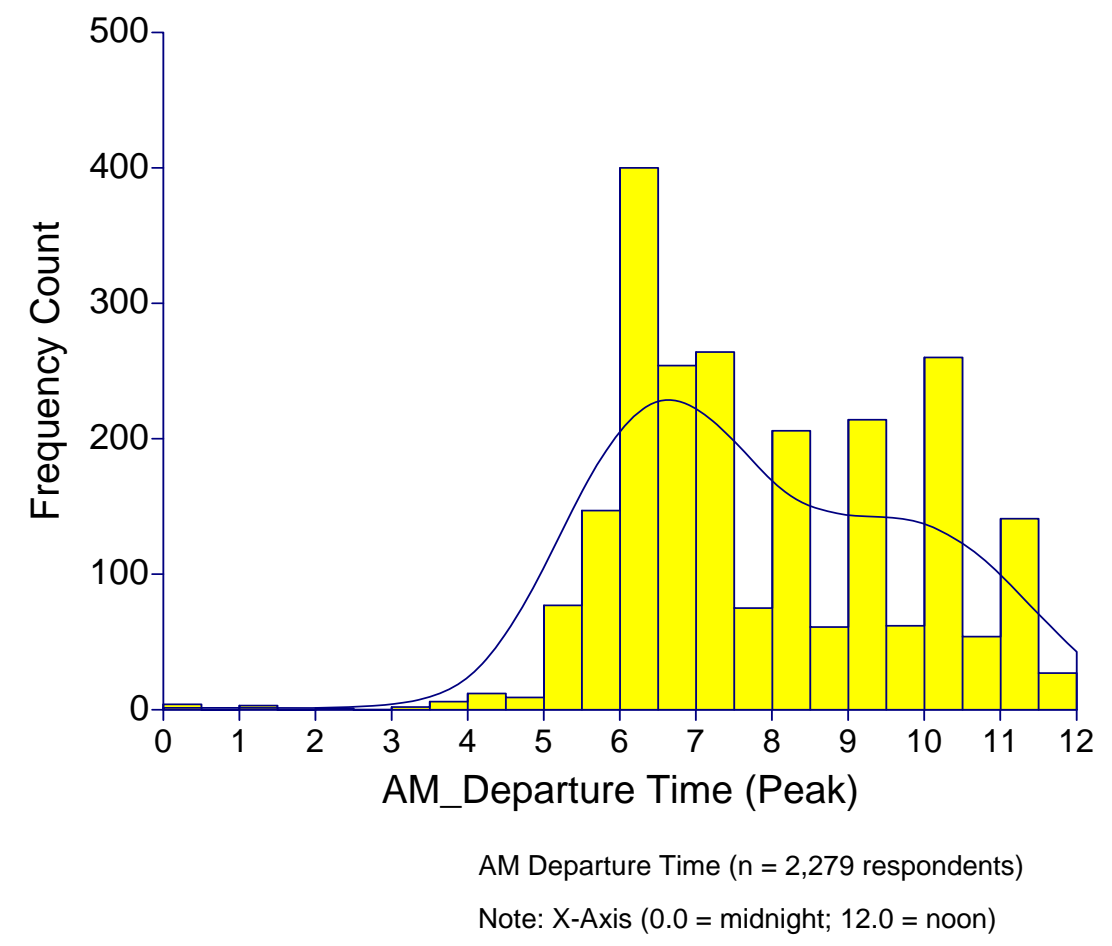

Figure 10b. Histogram of AM Departure Time - Peak Season. 


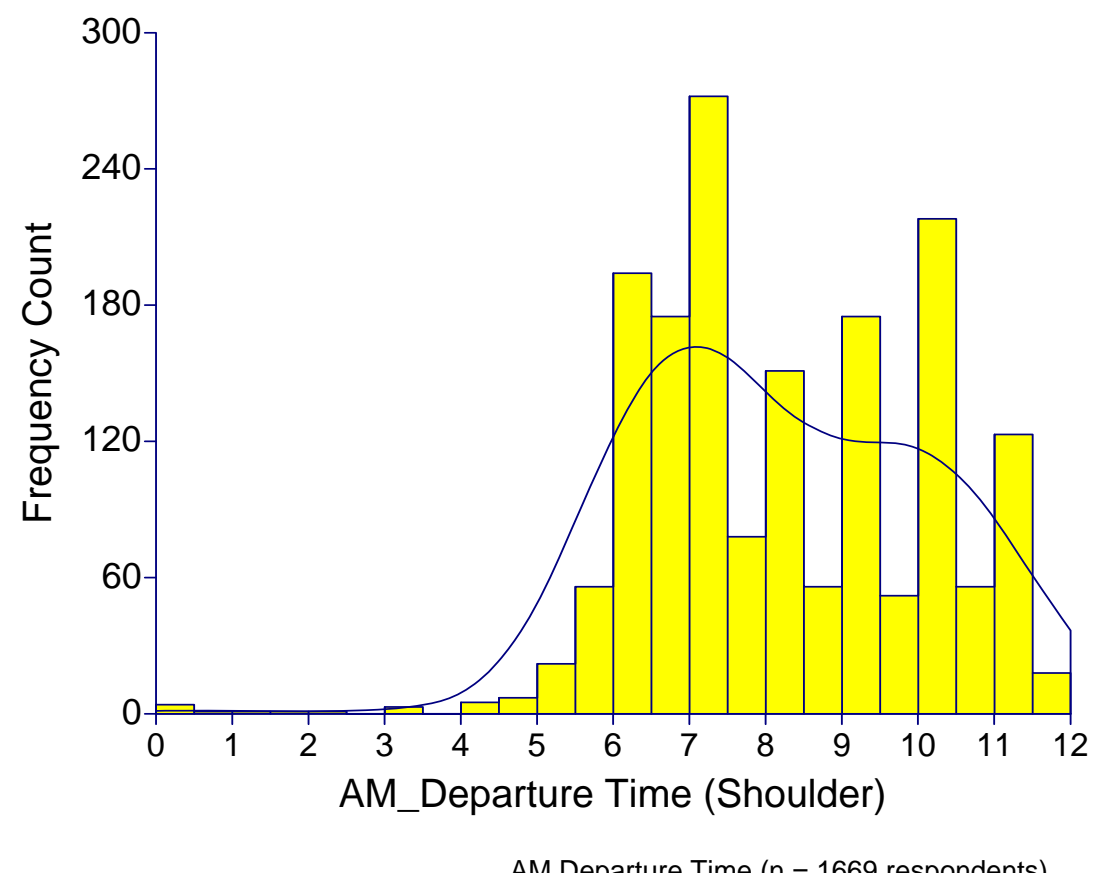

AM Departure Time $(n=1669$

Note: $\mathrm{X}$-Axis $(0.0=$ midnight; $12.0=$ noon $)$

Figure 10c. Histogram of AM Departure Time - Shoulder Season. 
Morning (AM) departure times by season and user group are highlighted in Figure 11. Substantial differences can be observed between the average AM departure times of Ramp users vs. those of other "late departure" groups. Notwithstanding, the seasonal trends in departure times are similar; with the earliest AM departure times occurring during the peak boating season and the latest AM departure times occurring during the off-peak season.

In general, boaters departing from Ramps tend to launch anywhere from an hour to 90 minutes earlier than boaters departing from Docks or Marina Wet Slips. In addition, boaters departing from Marina Dry Storage facilities tend to show the great seasonal variability in AM departure times.

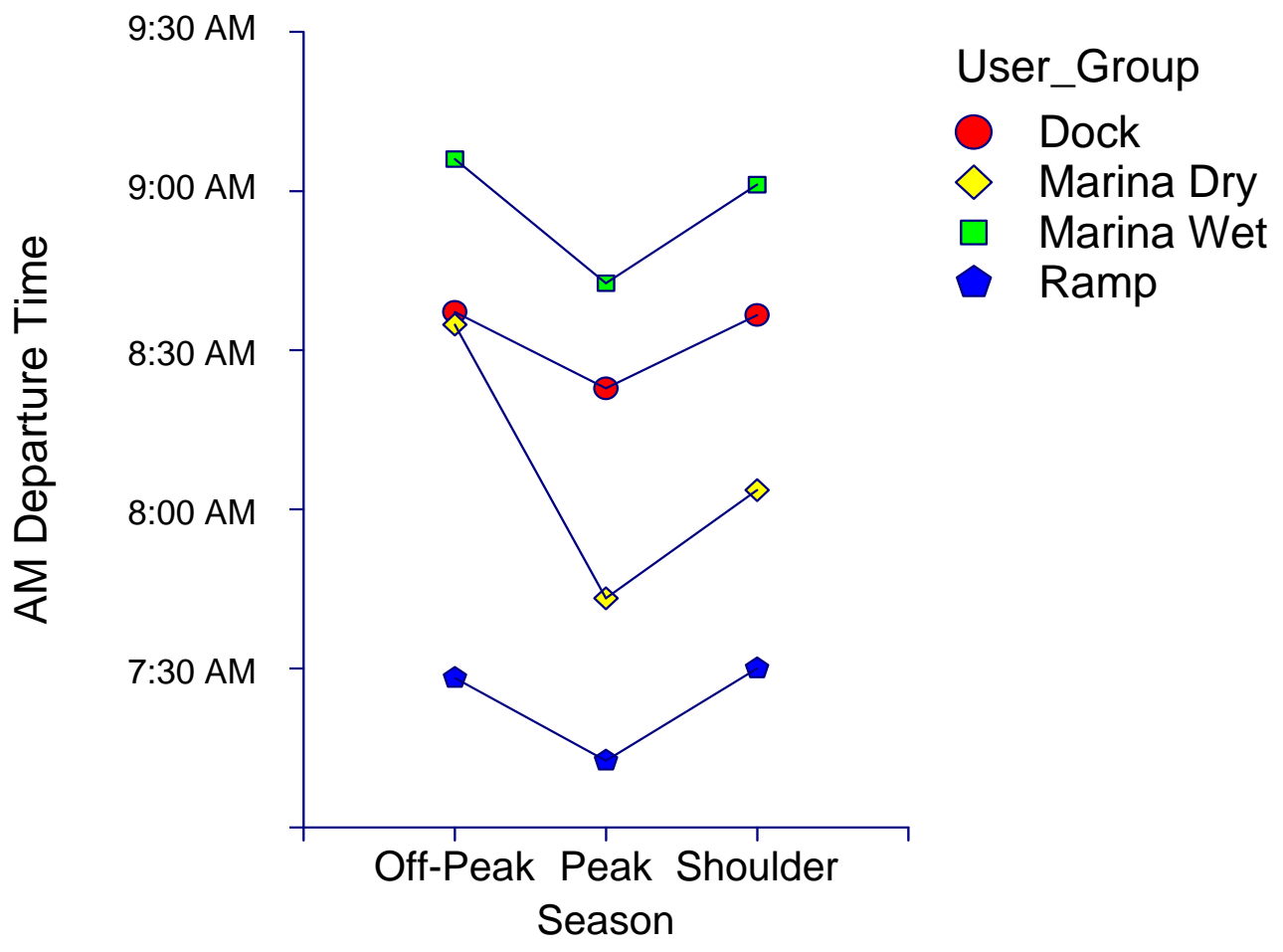

Figure 11. Mean AM Departure Times by User Group and Season. 


\section{PM Departure Time}

A total of $n=1,102$ survey respondents reported trips with PM departure times. This subsample represents roughly $17.4 \%$ of the total reported trips. The distribution of PM departure times is shown in Figure 12. The distribution is positively skewed and unimodal, with trip frequency counts that decline in a fairly consistent manner in later time slots. The peak PM departure time occurs between 12:00 noon and 1:00PM, followed by waves of staggered departures that occur with regularity (on the half hour, at 60 minute intervals) -- at 1:30PM, 2:30PM, 3:30PM, etc.

A seasonal summary of PM departure times by user groups is given in Table $25^{2}$. The average PM departure time is 2:35PM. While not listed, the median departure time is 2:00PM. The fact that the mean and median values are fairly similar suggests that the distribution of PM departure times is not severely skewed.

The reported PM launch time for trips that occurred during the peak boating season (2:38PM) is not significantly different from the overall average PM launch time of 2:35PM.

Several distinctions in departure times are observed when broken down by user group. First, the reported afternoon departure time of respondents using Ramps was significantly later than other user groups during each of the three designated boating seasons; with an overall average PM departure time of 3:30PM. Second, Marina Wet Slip users and those departing from Marina Dry Storage facilities reported PM departure times that were significantly earlier than the other groups in virtually all seasons (with the exception of Marina Dry Storage users during the peak season). Lastly, respondents departing from Docks had PM launch times that were statistically similar to the overall average and seasonal average PM departure times for all groups.

\footnotetext{
${ }^{2}$ Caution should be exercised in analyzing the trends in PM launch times as described in this section, due to the fact that relatively small sample sizes were obtained for Marina Dry Storage facility users, making statistical comparisons more difficult.
} 


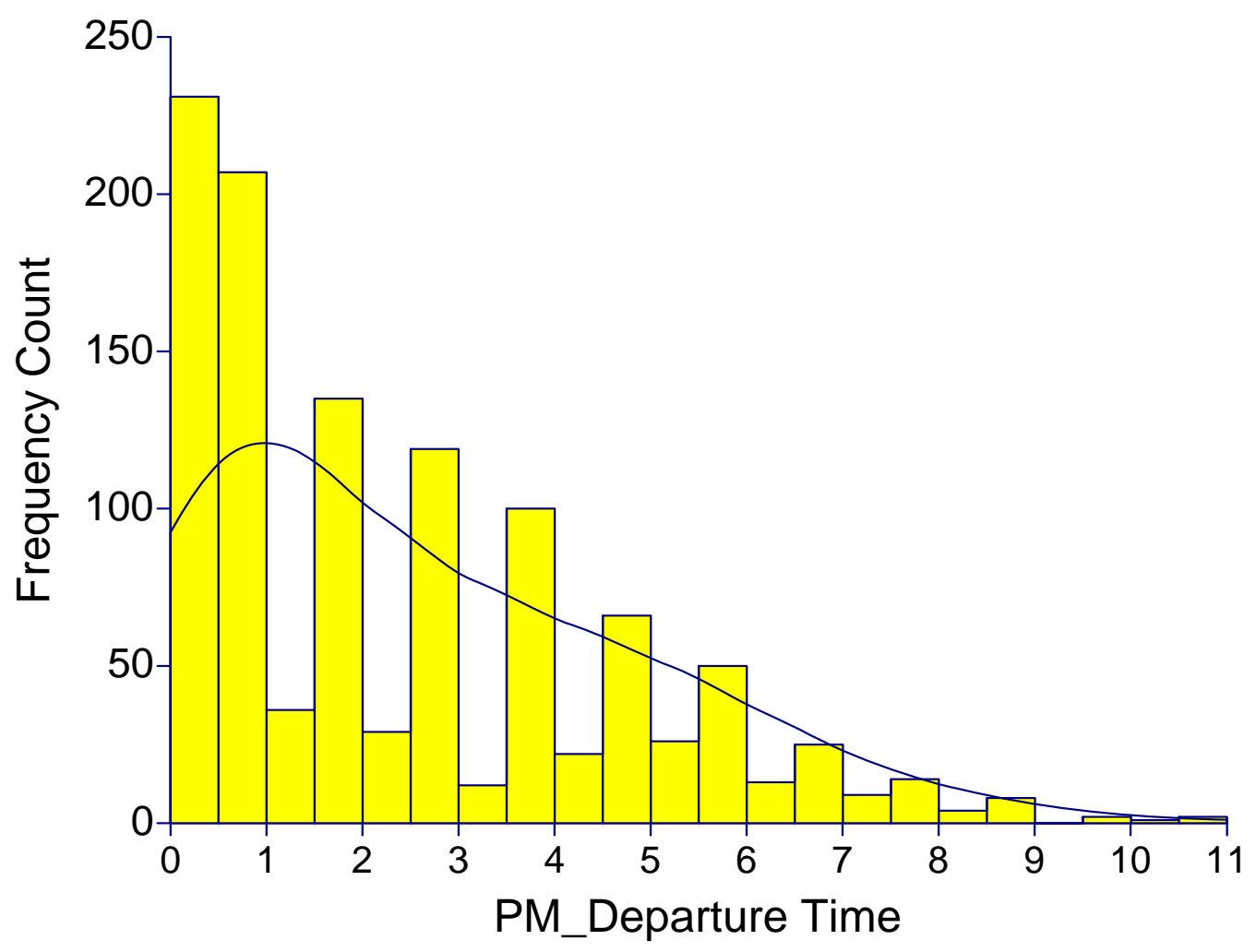

Departure Time $(n=1,111)$

Note: $X$-Axis (0.0 = noon; $11.0=11 \mathrm{PM}$ maximum)

Figure 12. Frequency Distribution of PM Launch Times for Reported Trips. 
Table 25. Mean PM Departure Time by Season and User Group.

\begin{tabular}{|c|c|c|c|c|c|}
\hline Season & All Users & Ramp & Dock & $\begin{array}{l}\text { Marina } \\
\text { Wet }\end{array}$ & $\begin{array}{l}\text { Marina } \\
\text { Dry }\end{array}$ \\
\hline Off-Peak & $\begin{array}{c}2: 32 P M \\
(246)\end{array}$ & $\begin{array}{c}4: 06 \dagger † * * \\
(61)\end{array}$ & $\begin{array}{l}2: 11 \dagger \\
(119)\end{array}$ & $\begin{array}{l}1: 48 \dagger^{*} \\
(54)\end{array}$ & $\begin{array}{c}1: 17 \dagger^{*} \\
(7)\end{array}$ \\
\hline Peak & $\begin{array}{c}2: 38 P M \\
(463)\end{array}$ & $\begin{array}{c}3: 33 \dagger \dagger \\
(127)\end{array}$ & $\begin{array}{l}2: 15 \dagger \\
(222)\end{array}$ & $\begin{array}{l}2: 17 \dagger \\
(100)\end{array}$ & $\begin{array}{c}2: 48^{* *} \\
(5)\end{array}$ \\
\hline Shoulder & $\begin{array}{c}2: 35 P M \\
(393)\end{array}$ & $\begin{array}{c}\text { 3:05††* } \\
(111)\end{array}$ & $\begin{array}{l}2: 24 \\
(206)\end{array}$ & $\begin{array}{c}2: 18 \dagger \\
(64)\end{array}$ & $\begin{array}{c}1: 07 \dagger^{*} \\
(8)\end{array}$ \\
\hline $\begin{array}{c}\text { All } \\
\text { Seasons }\end{array}$ & $\begin{array}{c}2: 35 P M \\
(\mathrm{n}=1,102)\end{array}$ & $\begin{array}{c}3: 30 P M+\dagger \\
(299)\end{array}$ & $\begin{array}{c}2: 17 P M \\
(547)\end{array}$ & $\begin{array}{c}2: 13 P M+ \\
(218)\end{array}$ & $\begin{array}{c}1: 36 P M \dagger \\
(20)\end{array}$ \\
\hline
\end{tabular}

Note: Mean departure time shown in boldface type; Sample sizes are shown in parentheses.

Note: All Users - represents all departure categories including Shoreline/Causeway and Other.

* Significantly less (earlier) than values observed from the same user group or the overall mean during other seasons at the $95 \%$ confidence;

** Significantly greater (later) than values observed for the same user group or the overall mean value during the other season at the $95 \%$ confidence level;

† Significantly less (earlier) than values observed for other user groups or the overall mean value during the same season at $95 \%$ confidence; and

$\dagger+$ Significantly greater (later) than values observed for other user groups or the overall mean value during the same season at $95 \%$ confidence. 


\section{Seasonal Analysis of Trip Duration}

Day Trips: Reported Trips of 24 Hours or Less

A seasonal comparison was made of trip durations of $n=5,655$ reported trips of 24 hours or less (representing roughly $90 \%$ of the total number of trips reported by survey respondents).

The analysis of day-trip duration revealed numerous statistical differences in the mean and median number of hours spent on the water during the three boating seasons and four user groups. Seasonal differences in the duration of day trips were the most pronounced between respondents departing from Ramps and Marina Dry Storage facilities (which tended to have the longest trip durations) and respondents departing from Docks (which tended to have the shortest day-trip durations).

Summary statistics for trip duration are presented in Table 26 for “day trips” (reported trips of 24 hours or less). Supporting graphics for this section are shown in Figures 13a and 13b.

Reported day trips for all users averaged about 5.72 hours, with an observed median daytrip duration of 5 hours. Median trip durations range from 4 to 7 hours, depending on the user category and season. Note that the $95 \%$ confidence interval for the mean day trip duration is between 5.62 hours and 5.82 hours for user groups across all seasons. The limited range of this interval (approximately .20 hours or 12 minutes) indicates that reported trip durations are compactly distributed about the mean of 5.72 hours and the median of 5 hours (a feature that is confirmed by the box plot of this distribution shown in Figure 13b).

Boaters departing from Marina Dry Storage facilities tended to stay out on the water anywhere from approximately 30 to 90 minutes longer than boaters from other categories during the peak and shoulder seasons. Day trips taken by Marina Wet Slip users tend to be shorter in the off-peak and shoulder seasons than during the peak season, with a seasonal use pattern that was very similar to that of boaters departing from Ramps. The shortest overall trip durations were associated with boaters departing from Docks; particularly during the off-peak and shoulder seasons. Dock users tended to report day trips that were anywhere from approximately 1 to 1.5 hours shorter in duration than those reported by boaters from other user groups.

All in all, significant differences were observed across user groups and seasons. The results presented in Table 26 reinforce the notion that boaters associated with the various user groups constitute distinct statistical populations with use characteristics and trip durations that vary significantly across seasons. Marked differences in trip duration were found between the peak season (where day trips tend to be the longest), and the non-peak and shoulder seasons (where day trips are anywhere from approximately 30 minutes to 1.5 hours shorter), depending on the user group. 
Table 26. Mean and Median Trip Durations (in hours) by Season and User Group--Reported "Day Trips” (Trips $\leq 24$ Hours).

\begin{tabular}{|c|c|c|c|c|c|}
\hline Season & All Users & Ramp & Dock & $\begin{array}{l}\text { Marina } \\
\text { Wet }\end{array}$ & $\begin{array}{c}\text { Marina } \\
\text { Dry }\end{array}$ \\
\hline Off-Peak & $\begin{array}{c}5.27 \text { hrs } \\
5 \text { hrs } \\
(1,223)\end{array}$ & $\begin{array}{c}5.76 \\
6 \dagger \\
(629)\end{array}$ & $\begin{array}{c}4.37 \dagger \dagger \\
4 \dagger \dagger \\
(376)\end{array}$ & $\begin{array}{c}5.42 \\
4 \dagger \dagger \\
(156)\end{array}$ & $\begin{array}{c}5.45 \\
4.5 \\
(46)\end{array}$ \\
\hline Peak & $\begin{array}{c}6.06 \text { hrs } \\
6 \text { hrs } \\
(2,528)\end{array}$ & $\begin{array}{c}\mathbf{6 . 3 8}+* \\
6 \dagger \\
(1,421)\end{array}$ & $\begin{array}{c}5.21 \dagger \dagger \\
4 \dagger \dagger \\
(688)\end{array}$ & $\begin{array}{c}\mathbf{6 . 3 0} \\
5 \\
(261)\end{array}$ & $\begin{array}{c}6.82 \dagger \\
7 \dagger \\
(107)\end{array}$ \\
\hline Shoulder & $\begin{array}{c}5.57 \text { hrs } \\
5 \text { hrs } \\
(1,904)\end{array}$ & $\begin{array}{c}\mathbf{6 . 0 8} \dagger \\
6 \dagger \\
(1,011)\end{array}$ & $\begin{array}{c}\mathbf{4 . 5 9} \dagger \dagger \\
4 \dagger \dagger \\
(597)\end{array}$ & $\begin{array}{c}5.53 \\
4 \dagger \dagger \\
(204)\end{array}$ & $\begin{array}{c}6.59 \dagger^{*} \\
6 十^{*} \\
(74)\end{array}$ \\
\hline $\begin{array}{c}\text { All } \\
\text { Seasons }\end{array}$ & $\begin{array}{c}5.72 \mathrm{hrs} \\
5 \mathrm{hrs}\end{array}$ & $\begin{array}{c}6.15 \dagger \\
(3083)\end{array}$ & $\begin{array}{c}4.78+\dagger \\
(1682)\end{array}$ & $\begin{array}{c}5.84 \\
(637)\end{array}$ & $\begin{array}{l}6.48 \dagger \\
(229)\end{array}$ \\
\hline
\end{tabular}

Note: Mean shown in bold type; median in non-bold type. Sub-sample sizes are shown in parentheses.

Groupings for All Seasons and All Users also contain observations for Shoreline/Causeway and Other.

* Significantly greater (longer) than values observed from the same user group during other seasons at the $95 \%$ confidence level;

** Significantly less (shorter) than values observed from the same user group during other seasons at the $95 \%$ confidence level;

† Significantly greater (longer) than values observed in other user groups during the same season at the $95 \%$ confidence level; and

$\dagger \dagger$ Significantly less (shorter) than values observed in other user groups during the same season at the $95 \%$ confidence level. 


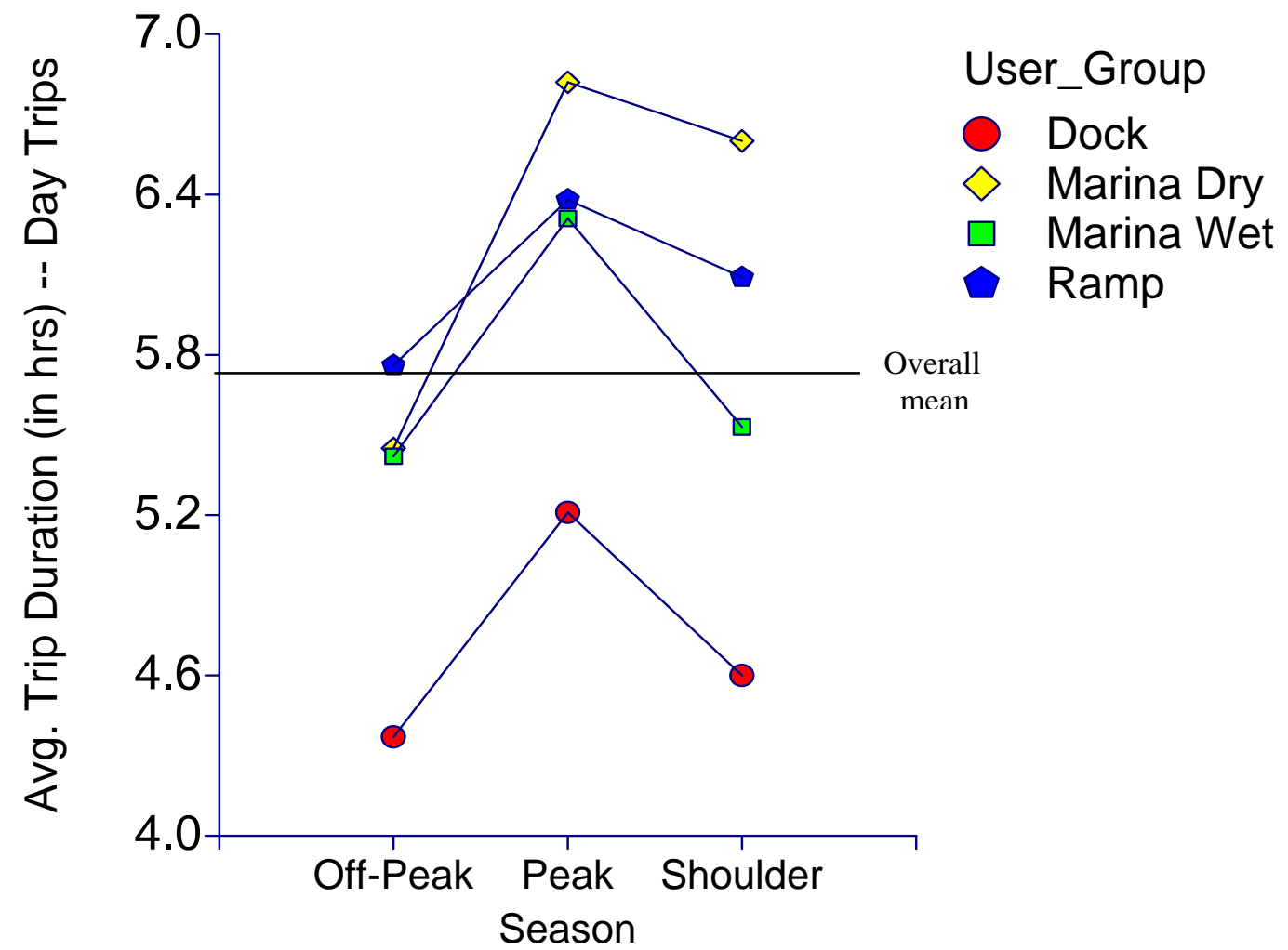

Figure 13a. Mean Reported Duration of Day Trips (in hours) by Season/User Group. 


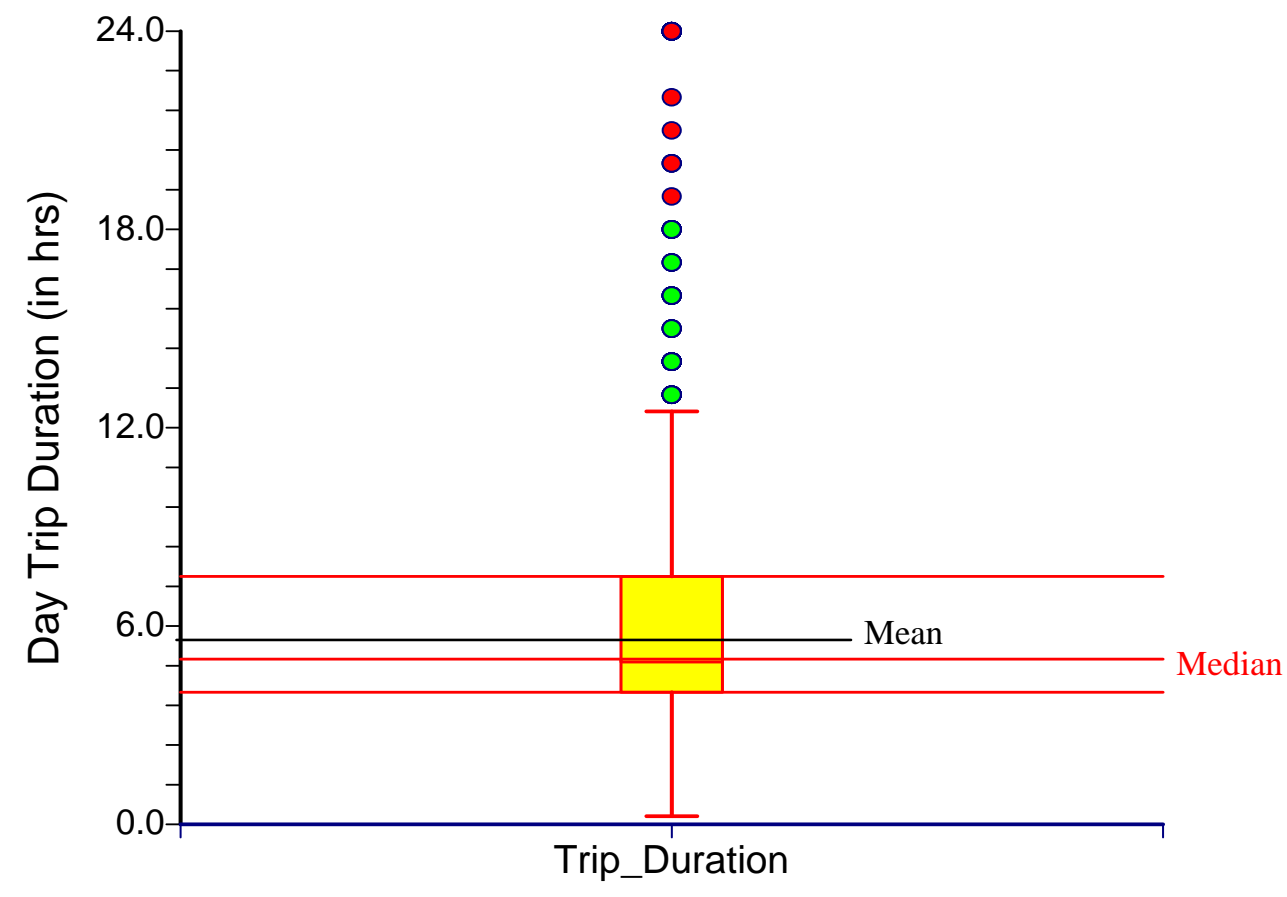

Figure 13b. Box-Plot of the Duration of "Day Trips" as Reported by Respondents from All User Groups. 
Overnighters: Reported Trips Greater than 24 Hours in Duration

A total of $n=717$ reported trips (or approximately $13.2 \%$ of the total trips reported) were of a duration that exceeded 24 hours. For convenience, these trips have been labeled as "overnighters" - or trips that are longer than 24 hours.

The average duration of reported overnighters was 121 hours, and the median overnight trip duration was 48 hours. The large gap between the mean and median values suggests that the distribution of overnight trip duration is highly skewed with the presence of extreme outlying values at the tail end of the distribution. An illustration of the distribution of reported over-night trip durations by season and user group is provided in Figure 14a.

It is interesting to note the substantial differences between respondents departing from Docks and Marina Wet Slips vs. those departing from Ramps and Marina Dry storage facilities. Dock and Marina Wet Slip users tended to report overnighters of durations that were three or more times in length than boaters departing from Ramps and Marina Dry Storage facilities. As stated earlier, this gap is likely due to the presence of large outlying values.

The maximum reported overnight trip duration for Dock users was 5,760 hours (240 days) and 4,320 hours (180 days) for Marina Wet Slip Users. Note that these values are far greater than the maximum reported trip durations of only 96 hours (4 days) and 336 hours (14 days), respectively, for respondents departing from Marina Dry Storage facilities and Boat Ramps. The large gap between these sub-groups suggests that Dock and Marina Wet Slip users tend to take longer over night trips, on average; yet their mean reported trip duration may be somewhat overstated (and unrepresentative of the typical overnight trip duration for boaters in these statistical populations) given the presence of numerous extreme observations or outliers. 


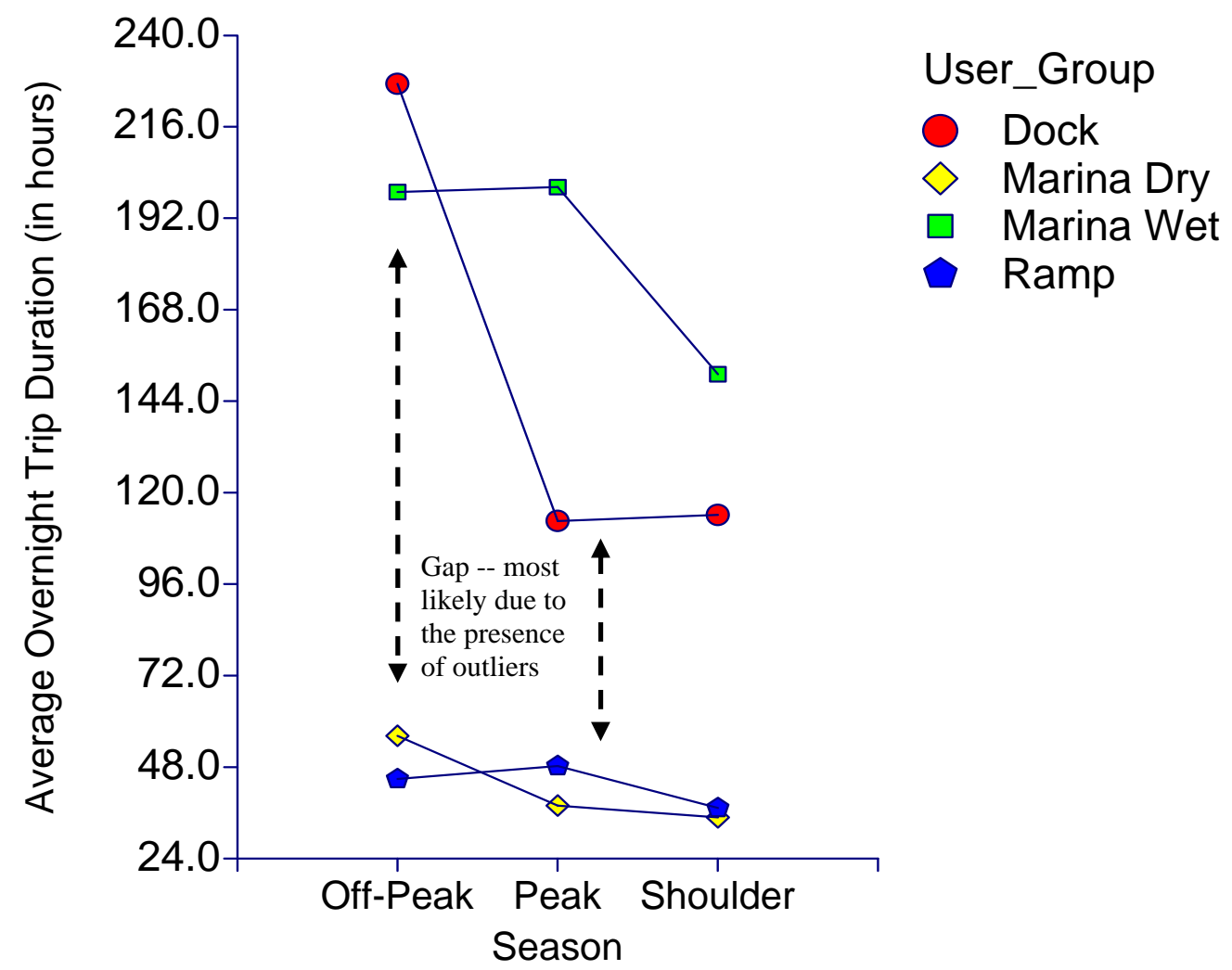

Note the gap between pairs of users groups - due to extreme outlying observations reported by users departing from Docks and Marina Wet Slips.

Figure 14a. Mean Reported Duration of Overnight Trips by Season and User Group (Trip duration $>24$ hours). 
In order to sidestep the influence of outliers, a separate analysis was carried out to compare overnight trips of more than 24 hours but less than or equal to 168 hours (7 days) in duration. Summary statistics for this "constrained" analysis are provided in Table 27, with results illustrated in Figures 14b and 14c. By eliminating the outliers, the mean overnight trip duration drops from 121 hours to 53.1 hours - a value that is more in line with the observed median value of 48 hours (see Box Plot in Figure 14c).

While a gap in trip duration between user groups was still evident in the constrained analysis (Figure 14b), the observed differences in trip duration values ranged between 12 and 24 hours, as opposed to the exaggerated gaps observed in Figure 14a (which were 3 or 4 times as large depending on the season, with the largest gaps occurring during the off-peak and peak seasons).

The constrained analysis revealed that Marina Wet Slip and Dock users still accounted for the longest trip durations across all seasons, with trip durations of approximately 56 to 64 hours (2.3 to 2.7 days) on average. Ramp and Marina Dry Storage users tend to have overnighters that averaged about 42 hours (1.75 days) in length, with Ramp users taking their longest overnighters during the peak season. Boaters departing from Marina Dry Storage facilities showed the greatest variation in trip duration across seasons, and tended to take relatively long overnighters during the off-peak season and relatively short trips during the shoulder season.

The longest overnighters were reported by Marina Wet Slip users, and were associated with trips that took place during the off-peak season. These overnighters, on average, were roughly 67 hours in duration (or 2.8 days); and were approximately 11 to 28 hours longer than trips taken by boaters from other user groups during this season. 
Table 27. Mean and Median Overnight Trip Durations (in hours) by Season and User Group--“Overnighters” (Trips > 24 Hours and $\leq 168$ Hours).

\begin{tabular}{|c|c|c|c|c|c|}
\hline Season & All Users & Ramp & Dock & $\begin{array}{l}\text { Marina } \\
\text { Wet }\end{array}$ & $\begin{array}{c}\text { Marina } \\
\text { Dry }\end{array}$ \\
\hline Off-Peak & $\begin{array}{c}53.51 \text { hrs } \\
48 \text { hrs } \\
(145)\end{array}$ & $\begin{array}{c}\mathbf{4 0 . 0 8} \dagger \dagger \\
33 \dagger \dagger \\
(60)\end{array}$ & $\begin{array}{c}57.52 \dagger \\
48 \\
(34)\end{array}$ & $\begin{array}{c}\mathbf{6 8 . 6 0} \dagger^{*} * \\
48 \\
(41)\end{array}$ & $\begin{array}{c}\text { 56.28* } \\
36+\dagger \\
(7)\end{array}$ \\
\hline Peak & $\begin{array}{c}55.78 \text { hrs } \\
48 \mathrm{hrs} \\
(243)\end{array}$ & $\begin{array}{c}\text { 48.33††* } \\
33 \dagger \\
(83)\end{array}$ & $\begin{array}{c}59.68 \dagger \\
48 \\
(63)\end{array}$ & $\begin{array}{c}\mathbf{6 0 . 5 5} \dagger \\
48 \\
(89)\end{array}$ & $\begin{array}{c}37.91 \dagger \\
31 \dagger \dagger \\
(6)\end{array}$ \\
\hline Shoulder & $\begin{array}{c}50.38 \text { hrs } \\
48 \text { hrs } \\
(255)\end{array}$ & $\begin{array}{c}37.03 \dagger \dagger \\
31 \dagger \dagger \\
(97)\end{array}$ & $\begin{array}{c}50.82 * * \\
48 \\
(57)\end{array}$ & $\begin{array}{c}64.94 \dagger \\
48 \\
(91)\end{array}$ & $\begin{array}{c}34.85 \dagger * * \\
33 \dagger \\
(7)\end{array}$ \\
\hline All & $\begin{array}{c}53.13 \text { hrs } \\
48 \text { hrs } \\
(649)\end{array}$ & $\begin{array}{c}\mathbf{4 1 . 8 1}+\dagger \\
32 \dagger \dagger \\
(240)\end{array}$ & $\begin{array}{c}55.95 \\
48 \\
(155) \\
\end{array}$ & $\begin{array}{c}63.73 \dagger \\
48 \\
(225) \\
\end{array}$ & $\begin{array}{c}42.83 \dagger \dagger \\
34 \dagger \dagger \\
(21)\end{array}$ \\
\hline
\end{tabular}

Note: Mean shown in bold type; median in non-bold type. Sub-sample sizes are shown in parentheses.

Groupings for All Seasons and All Users also contain observations for Shoreline/Causeway and Other.

* Significantly greater (longer) than values observed from the same user group during other seasons at the $95 \%$ confidence level;

** Significantly less (shorter) than values observed from the same user group during other seasons at the 95\% confidence level;

$\dagger$ Significantly greater (longer) than values observed in other user groups during the same season at the $95 \%$ confidence level; and

$\dagger †$ Significantly less (shorter) than values observed in other user groups during the same season at the $95 \%$ confidence level. 


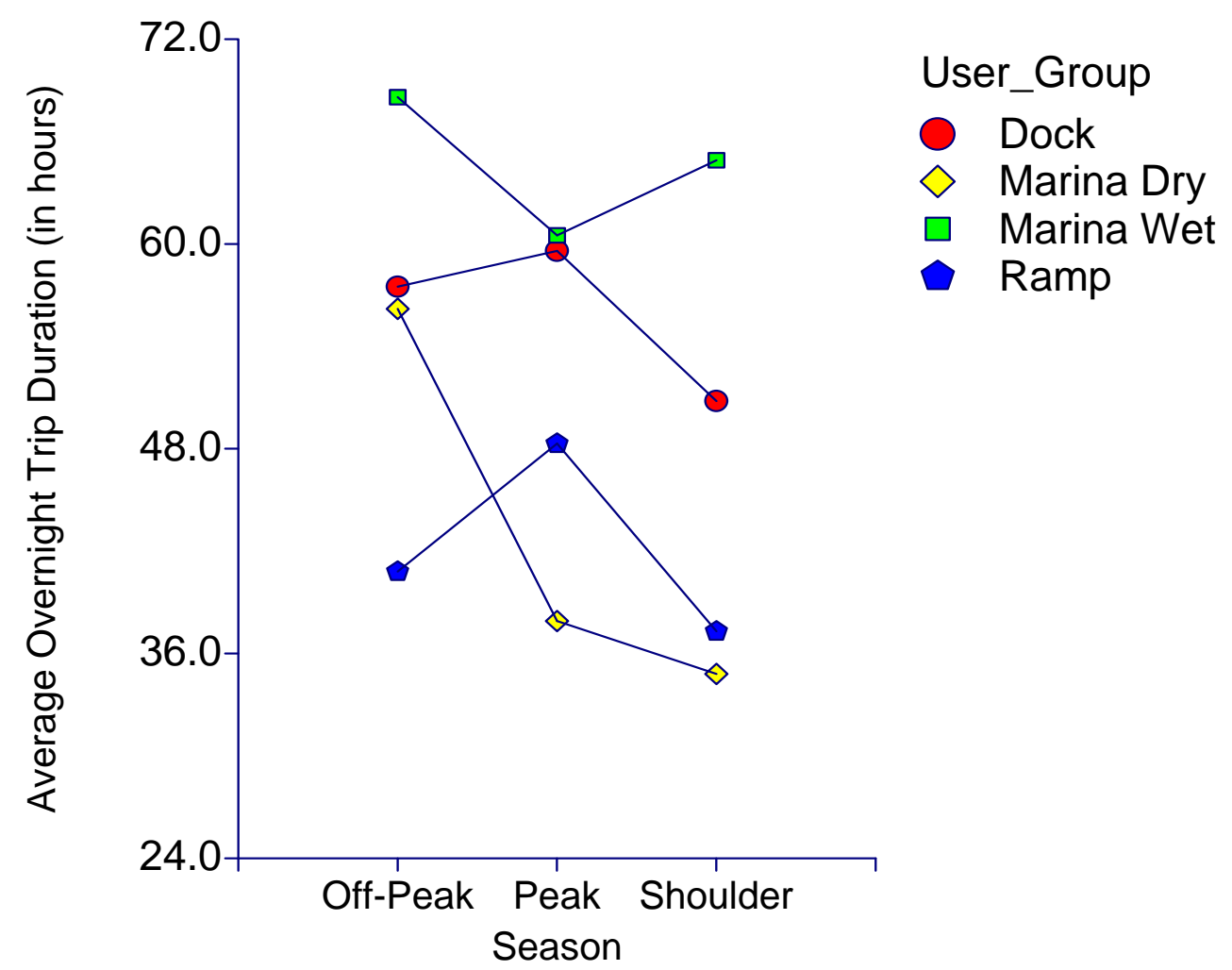

Figure 14b. Mean Reported Duration of Overnight Trips by Season and User Group (Trip duration $>24$ hours and $\leq 168$ hours). 


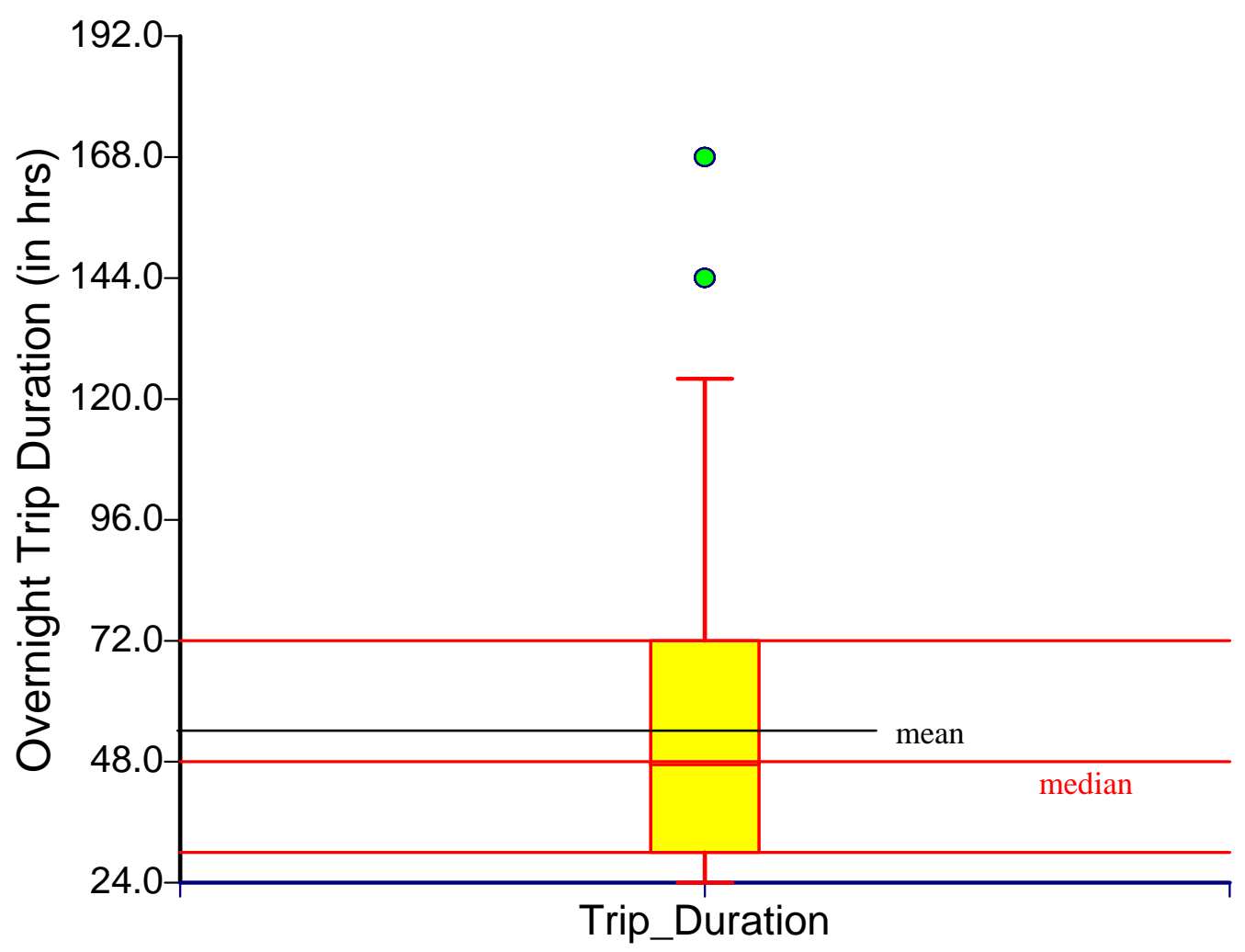

Figure 14c. Box Plot Showing Duration of Reported Overnight Trips by Survey Respondents from All User Groups (Duration $>24$ hours and $\leq 168$ hours). 


\section{Seasonal Analysis of Weekend vs. Weekday Trips}

Summary statistics highlighting the proportion of trips associated with weekend days (Saturday or Sunday) vs. weekdays (Monday through Friday) are based on the responses to Question 4 of the survey. Of the $n=4,955$ reported trips, 2,139 trips fell on weekend days yielding an overall proportion of .584. In other words, 58.4\% of the reported trips were classified as weekend trips and $41.6 \%$ were classified as weekday trips.

A breakdown of the proportion of weekend trips by user group and season is presented in Table 28. Ramp users had the highest proportion of reported trips falling on weekend days during the peak season -- .64 (or 64\%) -- a value that is significantly greater than the average for all user groups during the peak season. In addition, survey respondents departing from Ramps had higher percentages of weekend trips across all seasons, with an overall average of approximately $62 \%$ of their reported trips occurring during the weekend. Survey respondents departing from Docks had the second-highest overall percentage of trips occurring on weekends (approximately 56\%), with slightly over 57\% during the peak season. Boaters departing from Marina Dry Storage facilities tended to report the lowest percentage of weekend trips (with less than 50\% of their trips falling on weekend days). Moreover, respondents departing from Marina Dry Storage facilities had a very low percentage of weekend trips during the shoulder and off-peak seasons; with slightly less than $40 \%$ of reported trips occurring during the shoulder season and about $47 \%$ occurring during the off-peak season. Marina Wet Slip users reported that roughly 50\% of their trips taken during the off-peak and shoulder seasons occur on weekends. Almost 55\% of peak season trips reported by Marina Wet Slip took place on weekends.

The proportion of reported trips falling on weekend days during the peak boating season was .609 or approximately 61\%, on average, for all users. This value is significantly greater than the overall average proportion of $58.4 \%$ for all seasons. This suggests that peak-season boating trips are more likely to occur during the weekend in comparison to boat trips taken throughout the entire year or during the off-peak or shoulder seasons (where trips are more evenly divided between weekend days and weekdays).

It should be noted that if all days of the week were equally likely in terms of observing a trip (that is, trips were equally spread out over the course of the week), the "expected" proportion of weekend trips would be $2 / 7=.285$ (or 28.5\%). This represents a hypothetical benchmark by which to compare the proportion of reported trips by user group and/or season. In all cases, user groups posted proportions that significantly exceeded this benchmark in each identified boating season. The ratio of “reported" trips to “expected” trips -- $\mathbf{( 5 8 . 4 \%}$ / $\mathbf{2 8 . 5 \% )}$ or 2.05 - indicates that weekend trips are, on average, about twice as likely to occur than a weekday trip (across all seasons). During the peak season, this ratio increases to $(\mathbf{6 0 . 9 \%} / \mathbf{2 8 . 5 \% )}$ or 2.14 - suggesting an even greater likelihood of weekend trips. These indices highlight the weekend orientation of recreational boating trips in this study region. It should be noted that no user-group/season combination had a proportion of weekend trips that fell below the $28.5 \%$ benchmark. 
Table 28. Proportion of "Weekend Trips" by User Group and Season.

\begin{tabular}{|l|c|c|c|c|c|}
\hline Season & All Users & Ramp & Dock & Marina Wet & Marina Dry \\
\hline \multirow{2}{*}{ Off-Peak } & .397 & $.568^{*}$ & .360 & $.265^{* *}$ & $.334^{* *}$ \\
& $(2,399)$ & $(679)$ & $(758)$ & $(380)$ & $(565)$ \\
\hline \multirow{3}{*}{ Peak } & .526 & $.648^{* \dagger}$ &. $\mathbf{4 5 0 * * \dagger}$ & $.377^{* * \dagger}$ &. $\mathbf{4 8 6 * * \dagger}$ \\
& $(1,010)$ & $(376)$ & $(351)$ & $(90)$ & $(183)$ \\
\hline \multirow{3}{*}{ Shoulder } & .438 & $.658^{* \dagger}$ & $.380^{* *}$ & $.276^{* *}$ & $.289^{* * \dagger \dagger}$ \\
& $(714)$ & $(234)$ & $(234)$ & $(123)$ & $(121)$ \\
\hline \multirow{2}{*}{ Overall } & .431 & $.606^{*}$ & $.384^{* *}$ & $.274^{* *}$ & $.351^{* *}$ \\
& $\mathrm{~N}=4,955$ & $(1,561)$ & $(1,575)$ & $(744)$ & $(1,029)$ \\
\hline
\end{tabular}

Note: Sample and sub-sample sizes shown in parentheses.

* Significantly greater than mean for the same season at the $95 \%$ confidence level;

** Significantly less than mean for the same season at the $95 \%$ confidence level;

$\dagger$ Significantly greater than mean for same user group at the $95 \%$ confidence level; $\dagger \dagger$ Significantly less than mean for same user group at the 95\% confidence level. 


\section{Seasonal Analysis of Boating Activities}

Information on boating activities that occur on a typical trip was gathered from survey respondents, along with the number of typical trips taken per month. The objective was to identify the predominant boating activities by user group and season. A list of 15 boating activities was provided in the survey questionnaire (see Table 29 for a summary of those activities).

Survey respondents were asked to identify those boating activities that they engage in during a "typical trip" (Question 16). Moreover, boaters were asked to report the number of typical trips taken in each month of the year (Question 13). Data on boating activities and trips per month were combined to estimate the number, and more importantly, the percentage of specific trip activities that occur during each of the three designated boating seasons; taking into account the number of typical trips per month in a given season and reported trip activities that occur during a typical trip.

An estimated total number of trip activities was calculated for each of the three designated boating seasons based on information obtained from a sub-sample of $n=1,769$ survey respondents (the maximum number that responded to Questions 13 and 16). Table 30 provides a breakdown of the estimated percentage of recreational boating activities for each of the 15 activity categories listed in Question 16.

The percentage breakdown of activities tended to be fairly consistent from season to season. Fishing was the predominant activity of boaters who participated in the survey, accounting for approximately $19.0 \%$ of all activities that typically occur during a boating season. Fishing as a relative proportion of all activities exhibited a tendency to be only slightly higher during the non-peak season, accounting for about $19.3 \%$ of all typical activities. Nevertheless, as a typical activity, Fishing was almost twice as likely to occur as the next highest activity during each of the three boating seasons. Also high on the list of typical activities were Cruising (10.6\%), Nature Viewing (10.5\%), Sightseeing (8.8\%), and Socializing (8.4\%). The percentages of these activities were also fairly consistent across the three boating seasons.

It was noted in Chapter 5 that roughly 53\% of survey respondents cited Fishing as an activity in which they typically engage in. The lower percentage(s) of Fishing as a recreational boating activity depicted in this section may be a byproduct of two major factors: (a) boaters engaging in other activities (Cruising, Sightseeing, Nature Viewing, etc.) may have a greater propensity to take more trips per season (or per year) than do boaters engaging predominantly in Fishing; thereby lowering the overall percentage of Fishing relative to other activities; and (b) the fact that boaters tend to engage in several or numerous activities while on the water (a factor that would tend to also lower the overall percentage of Fishing as a typical activity that occurs during a given boating season). The findings in this section suggest that the survey instrument could be redesigned to solicit responses on the specific month in which the various boating activities occur, to gain even greater insight into seasonal use patterns. 
Table 29. A List of Boating Activities (as provided in the Survey)

\begin{tabular}{|c|l|c|l|}
\hline Activity Code & Activity Description & Activity Code & Activity Description \\
\hline FH & Fishing & OA & Overnight Anchoring \\
\hline SS & Sight Seeing & DA & Day Anchoring \\
\hline NV & Nature Viewing & DV & Diving \\
\hline BP & Beach Picnicking & JS & Jet Skiing \\
\hline BC & Beach Camping & CR & Cruising \\
\hline VR & Visiting Restaurants & SW & Swimming \\
\hline SO & Socializing & WS & Water Sports/Skiing \\
\hline SA & Sailing & & \\
\hline
\end{tabular}

Table 30. Percentage of Reported Boating Activities by Season (\% based on Typical Trip/Month/Season—weighted by number of trips)

\begin{tabular}{|c|c|c|c|c|}
\cline { 2 - 5 } \multicolumn{1}{c|}{} & \multicolumn{4}{c|}{ Seasons } \\
\hline $\begin{array}{c}\text { Activity } \\
\text { Code }\end{array}$ & $\boldsymbol{A l l}$ & Off-Peak & Peak & Shoulder \\
\hline FH & $\mathbf{1 9 . 0 4}$ & 19.33 & 19.14 & 18.80 \\
\hline SS & $\mathbf{8 . 8 4}$ & 8.86 & 8.92 & 8.75 \\
\hline NV & $\mathbf{1 0 . 4 5}$ & 10.89 & 10.40 & 10.24 \\
\hline BP & $\mathbf{5 . 6 2}$ & 5.46 & 5.66 & 5.69 \\
\hline BC & $\mathbf{2 . 5 7}$ & 2.72 & 2.59 & 2.45 \\
\hline VR & $\mathbf{6 . 5 6}$ & 6.42 & 6.51 & 6.67 \\
\hline SO & $\mathbf{8 . 4 5}$ & 8.30 & 8.39 & 8.58 \\
\hline SA & $\mathbf{3 . 0 8}$ & 3.70 & 3.02 & 2.77 \\
\hline OA & $\mathbf{3 . 9 4}$ & 4.38 & 3.81 & 3.79 \\
\hline DA & $\mathbf{5 . 5 2}$ & 5.48 & 5.46 & 5.59 \\
\hline DV & $\mathbf{3 . 7 0}$ & 3.62 & 3.72 & 3.73 \\
\hline JS & $\mathbf{0 . 3 2}$ & 0.25 & 0.38 & 0.36 \\
\hline CR & $\mathbf{1 0 . 5 8}$ & 10.35 & 10.61 & 10.71 \\
\hline SW & $\mathbf{6 . 4 0}$ & 5.97 & 6.44 & 6.61 \\
\hline WS & $\mathbf{4 . 9 0}$ & 4.26 & 4.95 & 5.23 \\
\hline Total & $\mathbf{9 9 . 9}$ & 99.9 & 100 & 99.9 \\
\hline
\end{tabular}

Note: Results based on $n=1,769$ respondents, and the reported number of "typical trips" per month (typical trips/month/season) and reported activities that are said to occur during a typical trip.

Column totals may not sum to $100 \%$ due to truncation/rounding of values.

Note: In all cases, the percentage values for activities in a given season were not statistically different from the percentage value shown for all seasons at the $95 \%$ confidence level. 


\section{Chapter 6. Perceived Detractors and Needs}

This chapter summarizes the responses to the following survey questions:

Question 24. "What detracts most from your boating experience?”

Question 25. "What is needed most to improve your boating experience?”

A typology of principal detractors (problems) and of principal needs (solutions to problems) was developed through a content analysis of the responses to these questions in $n=2,480$ returned surveys (i.e., the long version that contained Questions 24 and 25). Responses with shared general themes were grouped into primary categories for each question. In most cases, a primary category encompassed one or more subcategories, identified and extracted with as few as 4 or as many as 385 shared-theme responses. Every effort was made to capture the intended meaning of a given response and to maintain consistency in its assignment to a particular category/sub-category. Many survey respondents provided multiple answers to one or both questions and others chose not to answer, so that the total response number does not equal the returned survey count. Certain responses were excluded as not being amenable to intervention, such as weather issues or personal time/money concerns.

Given the differing numbers of mailed/returned surveys associated with the primary waterway access user groups (i.e., marina (wet slip and dry storage were combined) home dock (hereafter, “dock”), and ramp), and the potential for group-specific emphases with respect to detractors and needs, the analysis considered each group independently, as well as the total response base. Relative differences in priorities among the three were thereby defined, as well as the proportional contribution of each to the overall category response counts and rankings.

\section{Detractors}

Table 31 lists nine primary categories of boating detractors, identified through analysis of answers to Question 24. Each is followed by its composite subcategories. The leading primary category, accounting for one-third (33.4\%) of all analyzed responses $(n=2,720)$, addressed the lack of courtesy and/or seamanship in other boaters (i.e., failure to observe safe, considerate, or regulated boating practices). Responses citing excessive regulation, particularly as to speed control, made up the second highest detractor category, with $22.3 \%$ of total responses. Lack of access concerns, dominated by ramp issues and comprising $14.5 \%$ of the total, completed the top three primary categories. Similar numbers of responses cited environmental impacts $(9.6 \%$ of total), inadequate infrastructure ( $8.6 \%$ of total), or waterway congestion (6.3\% of total) as the principal detractor. A lack of destination provisions, from restaurants to water sport areas, was the focus of $2.2 \%$ of all responses. The last two primary categories, with nearly equal response numbers and together comprising the final 3.0\% of the total, indicated either that too little regulation or enforcement was a principal detractor or that nothing detracted from their boating 
Table 31. Primary Boating Detractors.

\begin{tabular}{|c|c|c|c|c|}
\hline Categories / Sub-Categories & $\begin{array}{c}\text { Totals } \\
\text { (all user groups) }\end{array}$ & $\begin{array}{c}\text { \% of } \\
\text { Total } \\
(n=2,720)\end{array}$ & $\begin{array}{c}\text { Category } \\
\text { Rank }\end{array}$ & $\begin{array}{c}\text { Subcategory } \\
\text { Rank } \\
\text { (top ten) }\end{array}$ \\
\hline Lack of Courtesy and/or Seamanship & 909 & 33.4 & 1 & \\
\hline Other Boater Behaviors in General & 385 & 14.2 & & 1 \\
\hline Ramp User Behaviors & 104 & 3.8 & & 8 \\
\hline PWCs & 207 & 7.6 & & 4 \\
\hline Speeding Power Boats & 114 & 4.2 & & 7 \\
\hline Large Boat Wakes & 58 & 2.1 & & \\
\hline Noisy Power Boats & 14 & 0.5 & & \\
\hline Boaters Under the Influence & 27 & 1.0 & & \\
\hline Excessive Regulation & 607 & 22.3 & 2 & \\
\hline Boating Regulations in General & 15 & 0.6 & & \\
\hline Fishing Regulations & 5 & 0.2 & & \\
\hline Manatee Zones & 311 & 11.5 & & 2 \\
\hline Speed Zones & 147 & 5.4 & & 6 \\
\hline No Wake Zones & 60 & 2.2 & & \\
\hline Excessive Security / Patrol Harassment & 69 & 2.5 & & \\
\hline Lack of Access & 395 & 14.5 & 3 & \\
\hline Public Ramps / Ramp Congestion & 258 & 9.5 & & 3 \\
\hline Ramp Parking & 68 & 2.5 & & \\
\hline Public Marinas / Slips & 55 & 2.0 & & \\
\hline Public Anchorage / Mooring & 14 & 0.5 & & \\
\hline Altered Environment & 262 & 9.6 & 4 & \\
\hline Shore Development, Loss of Natural Areas & 9 & 0.3 & & \\
\hline Dirty Water, Pollution & 100 & 3.7 & & 9 \\
\hline Shore / Island Trash & 68 & 2.5 & & \\
\hline Derelicts, Hurricane Debris & 15 & 0.6 & & \\
\hline Lack of Fish & 37 & 1.4 & & \\
\hline Grass Flats Destruction & 33 & 1.2 & & \\
\hline Infrastructure Shortcomings & 234 & 8.6 & 5 & \\
\hline Lack of Dredging (Shoaling) & 81 & 3.0 & & 10 \\
\hline Poor Channel Marks / Waterway Signs & 54 & 2.0 & & \\
\hline Inadequate Ramp Facilities & 64 & 2.4 & & \\
\hline Inadequate Marina Facilities (Pumpouts, Boatyards) & 23 & 0.8 & & \\
\hline Bridges / Port Locks & 12 & 0.4 & & \\
\hline Congestion & 170 & 6.3 & 6 & \\
\hline Waterways / Water-based Destinations & 170 & 6.3 & & 5 \\
\hline Lack of Destinations & 61 & 2.2 & 7 & \\
\hline Shore Facilities / Entertainment & 9 & 0.3 & & \\
\hline Waterfront Restaurants & 20 & 0.7 & & \\
\hline Public Dockage & 16 & 0.6 & & \\
\hline Designated Watersport Areas & 12 & 0.4 & & \\
\hline Beaches, Artificial Reefs & 4 & 0.1 & & \\
\hline Lack of Enforcement & 39 & 1.4 & 9 & \\
\hline Too Few Patrols & 5 & 0.2 & & \\
\hline Speed Zones & 8 & 0.3 & & \\
\hline Crab Trap Proliferation & 16 & 0.6 & & \\
\hline Fishing Regulations / Catch Limits & 10 & 0.4 & & \\
\hline No Detractors & 43 & 1.6 & 8 & \\
\hline
\end{tabular}


experience. (Note: "No detractors” was specifically stated, not inferred from a lack of response.) The relative contributions of the three different access groups to the primary detractor categories and their composite sub-categories are provided in Table 32. As in Table 31, the primary categories are listed in their overall descending rank, with a separate internal ranking of primary categories also shown for each user group.

The leading primary detractor category overall, "Lack of Courtesy and/or Seamanship,” encompassed a similar high percentage of marina and ramp user responses, with $34.3 \%$ and $38.2 \%$ of respective group totals, more than twice the number of the next leading category in each instance. For dock users the issue was also significant, ranking second in this user group with $23.4 \%$ of their total responses. The category contained all perceptions of unacceptable boating behavior, most often applied to bad boaters in general, making this the leading subcategory with $14.2 \%$ of the total question responses. Independently, specific operator groups (e.g., personal watercraft operators - PWC) were cited as well as specific practices, ranging from reckless behaviors (e.g., speeding or boating under the influence of alcohol) to ignorance of or noncompliance with established law ("rules of the road”). Concerns uniquely emphasized by a given access group included large boat wakes (5.5\% of marina responses) and inconsiderate behaviors at ramps (6.2\% of ramp responses). The latter stressed both delays in loading and offloading as well as exhibitions of impatience.

A preponderance of the dock user access group cited aspects of excessive regulation as leading detractors, with $40.4 \%$ of their total response count largely responsible for the overall second place ranking of this primary category. Within the marina and ramp populations, the category ranked fourth and third respectively, with $12.6 \%$ and $15.8 \%$ of group responses. Consistent among all three access groups was the leading subcategory of manatee zone regulation, subsuming $21.5 \%$ of dock responses, $5.6 \%$ of marina, and $7.9 \%$ of ramp, with at least twice the number of responses of the next highest subcategory, that of speed zones in general. Many answers dealt with a perceived inappropriate or non-science-based placement of the manatee zones, such as in waters too shallow. Recurring references were made to the imposition of manatee zones in Sykes Creek ( $n=9)$, Barge Canal $(n=9)$, and the Banana River $(n=4)$; similarly cited under the subcategories of excessive speed zones and no wake zones were Sykes Creek $(n=8)$, Barge Canal $(n=15)$, and the Banana River $(n=4)$. Inordinate time required to reach a boating destination was the chief detractor in 24 responses. Seven respondents indicated that manatee, speed, and/or no wake zones had ruined or markedly curbed recreational boating for their family. Also falling under excessive regulation and accounting for $2.5 \%$ of all detractor responses were concerns of overzealous marine law enforcement and security measures. Again, this subcategory drew relatively more responses from dock users (3.4\% of group total), followed by ramp users (2.5\% of group total). Complaints of patrol harassment included overlapping marine monitoring agencies ( $\mathrm{n}=5$ responses) and repetitive boardings/inspections ( $n=8$ responses). Ten responses were directed at overly restrictive security constraints in place at Port Canaveral.

The third largest category overall, lack of water access, drew more responses from marina (15.1\% of total and $3^{\text {rd }}$ in rank) and ramp (18.9\% of total and $2^{\text {nd }}$ in rank) users than from dock users (5.6\% of total and $5^{\text {th }}$ in rank). Expressions of too few ramps and prohibitive congestion at existing ramps were merged to form the largest subcategory ( $n=258$ overall 
responses, with 226 arising from ramp users). The lack of ramp parking, restricting access times of day and days of the week, was also a significant complaint of ramp users, garnering 3.9\% of this group response total. Similarly, the issue of too few marinas ranked highest in the marina access group, with $9.3 \%$ of their responses. The marina shortage was often (17 of the 55 total responses) expressed in terms of disappearing public marinas, losing out to development and privatization ("condo conversion”). Inadequate public anchorage and mooring provisions was also cited as an access deterrent, almost exclusively by marina users (3.0\% of marina total).

Negative environmental aspects to the boating experience made up the fourth largest detractor group overall, with similar rankings within the marina $\left(5^{\text {th }}\right)$, home dock $\left(4^{\text {th }}\right)$, and ramp $\left(4^{\text {th }}\right)$ access groups. Chief among the subcategories overall and also for each user group (4.5\% of marina, $4.7 \%$ of dock, and $3.0 \%$ of ramp user responses, with a total of $n=100$ responses) was dirty water and water trash. Independently, hurricane debris and derelicts in the water were singled out by small numbers in each user group ( $\mathrm{n}=15$ total responses). The presence of shore litter garnered the second highest number of responses in this category, both overall and for marina and dock users, and tied with water pollution among ramp users. Of note were 17 references to garbage on spoil islands. Fewer fish numbers ranked third in this category, with comparable percentages in all user groups, followed closely by grass flats destruction, for which all 33 responses came from ramp users (2.1\% of all ramp responses). The concern over shore development with loss of natural areas was expressed in a small number of answers $(n=9)$, including similar low percentages (0.3-0.5\%) in each user group.

Following environmental issues and ranking fifth overall in detractor response numbers was the primary category encompassing infrastructure shortcomings, with $8.6 \%$ of total responses. This category actually ranked second in the marina group and third in the home dock group, in both cases because of significant concern over lack of dredging (shoaling). This subcategory accounted for $7.1 \%$ of marina responses overall and $6.1 \%$ of dock responses. In comparison, only $0.4 \%$ of ramp user responses cited this problem. Targeted responses in this subcategory included poorly maintained residential canals and creeks ( $\mathrm{n}=13$ responses) and shoaling in the ICW ( $\mathrm{n}=5$ responses) and Banana River ( $\mathrm{n}=6$ responses) channels. Problems with existing ramp facility infrastructure made up the second highest subcategory, with $2.4 \%$ of all category responses. This was much more in evidence in ramp responses (4.5 times the relative occurrence in marina or dock responses). Deficiencies ranged from lighting or parking security to dock space, and maintenance issues included dirty, silted in, or broken ramps. Inadequate marina facilities encompassed $4.1 \%$ of all marina user responses ( $0.8 \%$ and $0.1 \%$ of dock and ramp responses respectively), touching principally upon the lack of haul-out, repair, or boatyard provisions, as well as the need for deeper water, storm protection, and pump-out stations. The final significant infrastructure subcategory entailed complaints of inadequate channel marks (e.g., in the Banana River) and confusing, poorly maintained, or hazardous waterway signs (particularly speed or manatee zone signs). Comprising 2.0\% of all responses, this detractor subcategory included a greater percentage from within the marina and dock responses than from ramp responses.

The detractor category addressing congestion did not lend itself to subcategory components. "Too many boaters" on the waterways and at water-based destinations was the sentiment expressed in 6.3\% of all answers to Question 24. Ranking sixth in the overall response 
count, it had greatest significance for the ramp user group, with 7.9\% of this response pool (fifth in rank). For dock users it ranked sixth (4.7\% of group total), and seventh for the marina using population (3.0\% of total).

Fewer response numbers identified recreational boating detractors with inadequate destination infrastructure, down to $2.2 \%$ of all responses and ranking seventh among the nine primary categories. On an internal percentage basis, this was of greatest concern for the marina group, with $4.5 \%$ of its responses, principally addressing the lack of waterfront restaurants, short term public dockage, and shore based amenities in general. The lack of waterfront restaurants also made up the largest subcategory for dock and ramp users, followed closely by the lack of designated water sport areas. The latter subcategory had an overlapping theme with manatee zone encumbrance, with thirteen responses citing loss of water sport (primarily water skiing) areas to manatee zones.

The final two primary categories shared similar response counts. The 43 responses proclaiming "no detractors" in their boating experience ranked 8 th overall, with an equal representation from the three user groups. Problems with lack of regulation subsumed only 39 responses, ninth in rank for all user groups. Failure to regulate crab trap proliferation encompassed the most responses ( $\mathrm{n}=16$ total), coming primarily from marina and dock users. Lack of enforcement of existing speed or fishing regulation was noted by small numbers within dock and ramp groups and within marina and ramp groups respectively, and the five perceptions of inadequate marine patrol numbers all came from ramp users. Many of these issues appear to have been more readily conveyed in terms of offender behavior (the leading primary category) than of failure to regulate or enforce.

Table 31 shows the top ten detractor subcategories, together accounting for 1,877 (or $69 \%$ ) of the $n=2,720$ total analyzed answers to Question 24. Some conclusions:

- Leading the list with $14.2 \%$ of total responses and drawing significantly from all user groups (highest subcategory for marina and ramp users, with 15.6 and 16.7 percent of respective totals, and ranking third for dock users, with $8.4 \%$ of total responses) was the subcategory citing the behaviors of other bad boaters in general. Lack of seamanship with respect to safe, considerate practices as well as competence was also the basis for three other top ten subcategories: PWCs, speeding power boats, and ramp behaviors. The first two of these, ranking fourth and seventh respectively, appeared to impact the boating experience of all user groups, whereas ramp users alone largely accounted for the $8^{\text {th }}$ place ranking of ramp behavior concerns.

- Likewise, the concern of this large ramp using group over access limitations posed by insufficient ramps drove its $3^{\text {rd }}$ place overall ranking.

- Under excessive regulation, the subcategories of too many and unreasonable manatee and speed zones ranked second and sixth overall, disproportionately originating from the dock users $\left(1^{\text {st }}\right.$ and $2^{\text {nd }}$ subcategories respectively for this access group).

- Waterway congestion ranked $5^{\text {th }}$, with greatest input from ramp users. 
- The environmental issue of dirty water received comparable input across access groups, ranking $9^{\text {th }}$ overall.

- Finally, from the infrastructure category, the lack of dredging completed the ten leading subcategories, with input primarily from marina and dock users for whom it ranked $3^{\text {rd }}$ and $4^{\text {th }}$ respectively. 
Table 32. Primary Detractors by Waterway Access Group.

\begin{tabular}{|c|c|c|c|c|c|c|c|c|c|}
\hline \multirow[b]{2}{*}{$\begin{array}{c}\text { BOATING DETRACTORS } \\
\text { CATEGORIES/SUBCATEGORIES }\end{array}$} & \multicolumn{3}{|c|}{ MARINA ACCESS } & \multicolumn{3}{|c|}{ DOCK ACCESS } & \multicolumn{3}{|c|}{ RAMP ACCESS } \\
\hline & Counts & $\begin{array}{c}\text { \% of all } \\
\text { Marina } \\
\text { Responses } \\
(n=397)\end{array}$ & $\begin{array}{l}\text { Marina } \\
\text { Category } \\
\text { Rank }\end{array}$ & Counts & $\begin{array}{c}\% \text { of all } \\
\text { Dock } \\
\text { Responses } \\
(\mathrm{n}=774)\end{array}$ & $\begin{array}{c}\text { Dock } \\
\text { Category } \\
\text { Rank }\end{array}$ & Counts & $\begin{array}{c}\% \text { of all } \\
\text { Ramp } \\
\text { Responses } \\
\text { (n=1549) }\end{array}$ & $\begin{array}{c}\text { Ramp } \\
\text { Category } \\
\text { Rank }\end{array}$ \\
\hline Lack of Courtesy and/or Seamanship & 136 & 34.3 & 1 & 181 & 23.4 & 2 & 592 & 38.2 & $\mathbf{1}$ \\
\hline Bad Boater Behaviors in General & 62 & 15.6 & & 65 & 8.4 & & 258 & 16.7 & \\
\hline Ramp Users & 4 & 1.0 & & 4 & 0.5 & & 96 & 6.2 & \\
\hline PWCs & 20 & 5.0 & & 54 & 7.0 & & 133 & 8.6 & \\
\hline Speeding Power Boats & 24 & 6.0 & & 35 & 4.5 & & 55 & 3.6 & \\
\hline Large Boat Wakes & 22 & 5.5 & & 11 & 1.4 & & 25 & 1.6 & \\
\hline Noisy Power Boats & 0 & 0 & & 6 & 0.8 & & 8 & 0.5 & \\
\hline Boaters Under the Influence & 4 & 1.0 & & 6 & 0.8 & & 17 & 1.1 & \\
\hline Excessive Regulation & 50 & 12.6 & 4 & 313 & 40.4 & 1 & 244 & 15.8 & 3 \\
\hline Boating Regulations in General & 7 & 1.8 & & 5 & 0.6 & & 3 & 0.2 & \\
\hline Fishing Regulations & 1 & 0.3 & & 0 & 0 & & 4 & 0.3 & \\
\hline Manatee Zones & 22 & 5.6 & & 166 & 21.5 & & 123 & 7.9 & \\
\hline Speed Zones & 10 & 2.5 & & 83 & 10.7 & & 54 & 3.5 & \\
\hline No Wake Zones & 5 & 1.3 & & 33 & 4.3 & & 22 & 1.4 & \\
\hline Excessive Security, Patrol Harassment & 5 & 1.3 & & 26 & 3.4 & & 38 & 2.5 & \\
\hline Lack of Access & 60 & 15.1 & 3 & 43 & 5.6 & 5 & 292 & 18.9 & 2 \\
\hline Public Ramps/Ramp Congestion & 10 & 2.5 & & 22 & 2.8 & & 226 & 14.6 & \\
\hline Ramp Parking & 1 & 0.3 & & 6 & 0.8 & & 61 & 3.9 & \\
\hline Public Marinas/Slips & 37 & 9.3 & & 14 & 1.8 & & 4 & 0.3 & \\
\hline Public Anchorages/Moorings & 12 & 3.0 & & 1 & 0.1 & & 1 & 0.1 & \\
\hline Altered Environment & 38 & 9.6 & 5 & 67 & 8.7 & 4 & 157 & 10.1 & 4 \\
\hline Shore Development/Loss of Nat. Areas & 1 & 0.3 & & 4 & 0.5 & & 4 & 0.3 & \\
\hline Dirty Water, Pollution & 18 & 4.5 & & 36 & 4.7 & & 46 & 3.0 & \\
\hline Shore/Island Trash & 9 & 2.3 & & 13 & 1.7 & & 46 & 3.0 & \\
\hline Derelicts, Hurricane Debris & 6 & 1.5 & & 5 & 0.6 & & 4 & 0.3 & \\
\hline Lack of Fish & 4 & 1.0 & & 9 & 1.2 & & 24 & 1.5 & \\
\hline Grass Flats Destruction & 0 & 0 & & 0 & 0 & & 33 & 2.1 & \\
\hline Infrastructure Shortcomings & 66 & 16.6 & 2 & 88 & 11.4 & 3 & 80 & 5.2 & 6 \\
\hline Lack of Dredging (Shoaling) & 28 & 7.1 & 3 & 47 & 6.1 & & 6 & 0.4 & \\
\hline Channel Marks/Waterway Signs & 14 & 3.6 & & 23 & 3.0 & & 17 & 1.1 & \\
\hline Inadequate Ramp Facilities & 3 & 0.8 & & 6 & 0.8 & & 55 & 3.6 & \\
\hline Inadequate Marina Facilities & 16 & 4.1 & & 6 & 0.8 & & 1 & 0.1 & \\
\hline Bridges, Port Locks & 5 & 1.3 & & 6 & 0.8 & & 1 & 0.1 & \\
\hline Congestion & 12 & 3.0 & 7 & 36 & 4.7 & 6 & 122 & 7.9 & 5 \\
\hline Waterways/Water-based Destinations & 12 & 3.0 & & 36 & 4.7 & & 122 & 7.9 & \\
\hline Lack of Destinations & 18 & 4.5 & 6 & 21 & 2.7 & 7 & 22 & 1.4 & 7 \\
\hline Shore Amenities, Entertainment Fac. & 4 & 1.0 & & 2 & 0.3 & & 3 & 0.2 & \\
\hline Waterfront Restaurants & 6 & 1.5 & & 7 & 0.9 & & 7 & 0.5 & \\
\hline Public Dockage & 6 & 1.5 & & 6 & 0.8 & & 4 & 0.3 & \\
\hline Designated Watersport Areas & 1 & 0.3 & & 6 & 0.8 & & 5 & 0.3 & \\
\hline Beaches, Artificial Reefs & 1 & 0.3 & & 0 & 0 & & 3 & 0.2 & \\
\hline Lack of Enforcement & 10 & 2.5 & 8 & 11 & 1.4 & 9 & 18 & 1.2 & 9 \\
\hline Too Few Patrols & 0 & 0 & & 0 & 0 & & 5 & 0.3 & \\
\hline Speed Zones & 0 & 0 & & 5 & 0.6 & & 3 & 0.2 & \\
\hline Crab Trap Proliferation & 8 & 2.0 & & 6 & 0.8 & & 2 & 0.1 & \\
\hline Fishing Regulations/Catch Limits & 2 & 0.5 & & 0 & 0 & & 8 & 0.5 & \\
\hline No Detractors & 7 & 1.8 & 9 & 14 & 1.8 & 8 & 22 & 1.4 & 7 \\
\hline
\end{tabular}




\section{Needs}

Analysis of responses to Question 25 also suggested nine primary categories, most with one or more component subcategories, as listed in Table 33. As expected, responses addressing solutions to problems (i.e., needs) tended to have themes mirroring those of the problems themselves (i.e., detractors). However, the areas of emphasis shifted in hierarchy somewhat, both overall and within the three access groups (see Table 34). The perception of bad boater behaviors under detractors, for example, was not offset by the same preponderance of regulation enforcement or education measures under needs. Rather, the leading primary category was for more water access, garnering $26 \%(n=696)$ of the total response number $(n=2,682)$. Whereas infrastructure shortcomings ranked $5^{\text {th }}$ overall under detractors, infrastructure improvement suggestions made up $17.6 \%(n=473)$ of all needs responses, making it second in rank. Following closely with $16.9 \%$ was the need for less regulation, commanding six percent more of the response total than the fourth ranked category, which addressed the need for more regulation or enforcement. In descending order, the next three primary categories, each garnering a similar number of responses, were environmental improvement (8.5\% of total), the provision of more boating destination infrastructure ( $8.4 \%$ of total), and boater education (7.7\%). Although complaints of congested waterways made up 6.3\% of all responses to Question 24, the need for less congestion comprised only $1.4 \%$ of responses, making it the smallest category and trailing the $2.6 \%$ indicating "no needs" in their boating experience.

The water access issue led among primary categories because of the numbers expressing a need for more ramps. This dominated in responses from the ramp access group, with $21 \%$ of its total responses, but the subcategory also ranked fourth among dock users and fifth in the marina group. Repeatedly mentioned areas of insufficient ramp numbers included South Brevard County ( $n=29$ responses), the Pineda Causeway area of the Banana River ( $n=10$ responses), Port Canaveral $(n=9)$, and the Indian River side of Merritt Island $(n=6)$. The second most responses (5.9\% of total) under access needs focused on ramp parking, again largely contributed by ramp users (9.3\% of group). This was followed by the need for more marinas, slips, and dry storage, with $4.4 \%$ of total responses driven by the $18 \%$ of all marina user responses and making it the dominant needs subcategory for this user group. Consistent with the concern over disappearing marinas under detractors, the stressed need was for public marinas, stopping the loss to private condos, and curbing the rise in slip fees. Waterway access needs entailing moorings and anchorages also arose primarily from the marina users (4.9\% of group).

The subcategory calling for improved ramp facilities garnered 3 times the relative percentage of answers to Question 25 as its detractor counterpart to Question 24 (7.2\% vs. 2.4\%), such that it ranked first under the need for infrastructure improvements, itself the second leading primary category overall and for each user group. Within the litany of ramp improvement needs (Note: ramp parking was considered separately, under access needs), those garnering more than 10 responses included better maintenance $(n=29$, particularly as to sand build-up), better security and lighting $(n=21)$, more dock space for tie-ups $(n=12)$, bathroom facilities $(n=11)$, freshwater wash down availability ( $\mathrm{n}=11)$, and enlarged (wider, longer, deeper) ramps $(\mathrm{n}=19)$. The need for dredging ranked second in this category (4.2\% overall), chiefly an issue for marina and dock users and emphasizing residential canals $(n=16)$ and channels in general $(n=19$, of 
Table 33. Primary Boater Needs.

\begin{tabular}{|c|c|c|c|c|}
\hline Categories / Sub-Categories & $\begin{array}{c}\text { Totals } \\
\text { (all user groups) }\end{array}$ & $\begin{array}{c}\text { \% of Total } \\
(\mathrm{n}=2,682)\end{array}$ & $\begin{array}{c}\text { Category } \\
\text { Rank }\end{array}$ & $\begin{array}{c}\text { Subcategory Rank } \\
\text { (top ten) }\end{array}$ \\
\hline More Water Access & 696 & 26.0 & 1 & \\
\hline For Boaters in General & 15 & 0.6 & & \\
\hline Ramps & 377 & 14.1 & & 1 \\
\hline Ramp Parking & 157 & 5.9 & & 4 \\
\hline Marinas/Slips/Dry Storage & 119 & 4.4 & & 7 \\
\hline Anchorages and Public Moorings & 28 & 1.0 & & \\
\hline Infrastructure Improvements & 473 & 17.6 & 2 & \\
\hline Channel Marks / Waterway Signs & 105 & 4.0 & & 9 \\
\hline Dredging & 112 & 4.2 & & 8 \\
\hline Ramp Facilities & 192 & 7.2 & & 3 \\
\hline Full Service Marinas & 29 & 1.1 & & \\
\hline Pumpout Stations & 9 & 0.3 & & \\
\hline Fuel Docks & 15 & 0.6 & & \\
\hline Bridges, Port Locks & 11 & 0.4 & & \\
\hline Less Regulation & 454 & 16.9 & 3 & \\
\hline Government Regulation in General & 22 & 0.8 & & \\
\hline Patrol Harassment & 13 & 0.5 & & \\
\hline Speed zones & 140 & 5.2 & & 6 \\
\hline No Wake Zones & 45 & 1.7 & & \\
\hline Manatee Zones & 212 & 7.9 & & 2 \\
\hline Fishing Regulations & 11 & 0.4 & & \\
\hline Canaveral Security & 11 & 0.4 & & \\
\hline More Regulation / Better Enforcement & 291 & 10.9 & 4 & \\
\hline Boating Regulations in General & 56 & 2.1 & & \\
\hline Operator Licensure & 43 & 1.6 & & \\
\hline PWC Regulation & 33 & 1.2 & & \\
\hline Powerboat Regulation & 10 & 0.4 & & \\
\hline Speed Zone Enforcement & 42 & 1.5 & & \\
\hline More Pole/Troll Zones & 24 & 0.9 & & \\
\hline More Patrol Presence on Waterways & 38 & 1.4 & & \\
\hline Ramp Supervision & 17 & 0.6 & & \\
\hline Drinking \& Boating & 7 & 0.3 & & \\
\hline Recreational \& Commercial Fishing & 16 & 0.6 & & \\
\hline Crab Traps & 5 & 0.2 & & \\
\hline Environmental Protection & 228 & 8.5 & 5 & \\
\hline Improved Water Quality & 85 & 3.2 & & 10 \\
\hline Less Island / Shore Trash & 19 & 0.7 & & \\
\hline Sunken Boats, Derelicts Removal & 20 & 0.7 & & \\
\hline More Fish & 62 & 2.3 & & \\
\hline Less Shore Development / More Natural Areas & 27 & 1.0 & & \\
\hline Grass Flats Protection & 15 & 0.6 & & \\
\hline More Destinations/Activity Provisions & 226 & 8.4 & 6 & \\
\hline Public Places to Visit & 13 & 0.5 & & \\
\hline Waterside Restaurants & 66 & 2.5 & & \\
\hline Public and Transient Dockage & 46 & 1.7 & & \\
\hline Beaches, Parks & 29 & 1.1 & & \\
\hline Designated Watersport Areas & 43 & 1.6 & & \\
\hline Artificial Reefs & 29 & 1.1 & & \\
\hline Boater Education & 207 & 7.7 & 7 & \\
\hline Safety/Etiquette/Boating Skills/Regulations & 151 & 5.6 & & 5 \\
\hline Environmental Stewardship & 20 & 0.7 & & \\
\hline PWC Education & 9 & 0.3 & & \\
\hline Ramp Launch \& Retrieval & 27 & 1.0 & & \\
\hline Less Congested Waterways & 37 & 1.4 & 9 & \\
\hline Fewer Boaters & 37 & 1.4 & & \\
\hline No Needs & 70 & 2.6 & 8 & \\
\hline
\end{tabular}


which 8 cited the Intra-Coastal Waterway - ICW). In addition, there were 13 answers desirous of a new inlet's being dredged, citing faster ocean access and improved water flushing. This subcategory was followed closely by better channel marks and waterway signs (4.0\% overall). The need for more well lit channel markers was conveyed in 13 responses, and the Banana River was most often cited as needing markers $(n=10)$. Signage concerns emphasized clearer and less ambiguous speed restriction signs. The need for full service marinas centered upon haulout and boatyard provisions for boat servicing. While marina users were more represented in this subcategory, the separate consideration of waterway fuel docks received relatively more input from dock users, and both groups largely accounted for the few responses citing more pumpout stations and more accommodating bridges and Port locks.

Ranking third overall, with $16.9 \%$ of all responses, was the primary category identified with less regulation. Consistent with Question 24 responses, the preponderance wanted less extensive and/or less strict manatee $(n=212)$, speed $(n=140)$, and no wake zones $(n=45)$. The issue of manatee zones elicited recurring calls for more science-based, more seasonal, more judicious, or more balanced application. While it was the dominant subcategory for dock users, with $14.5 \%$ of the group total, less manatee zone regulation also drew $3.4 \%$ of all marina responses and 5.9\% of ramp responses. Less governmental boating regulation in general was the $4^{\text {th }}$ highest subcategory ( $0.8 \%$ of total responses), followed by smaller numbers for less patrol interference, an easing of Port Canaveral security, and fewer fishing regulations.

Responses in favor of more regulation/enforcement were more widely distributed under different subcategories, each comprising at most 1-2\% of all responses but together making up the

$4^{\text {th }}$ largest category of needs. Among ramp users this category ranked third overall, compared to $6^{\text {th }}$ for both dock and marina users. Better enforcement of existing boating regulation in general was the leading subcategory, with $2.1 \%$ of all answers, followed by better speed zone enforcement (1.5\% of total), more comprehensive operator licensure (1.6\% of total), and more patrol presence on the water $(1.4 \%)$. While access group contributions were comparable overall, ramp users were the chief proponents of more pole/troll areas (1.6\% of group) and greater ramp supervision (1.0\% of group).

Environmental improvement and more boating activity provisions ranked $5^{\text {th }}$ and $6^{\text {th }}$ respectively, but with only two responses separating the total counts. Improved water quality comprised the largest environmental subcategory, with 3.2\% of the total spread over the three access groups, the highest internal percentage coming from dock users (4.5\% of group) followed by marina users (3.4\% of group). The other substantial subcategory, the need for greater fish populations, involved significant numbers from each access group, but with ramp user preponderance (3.2\% of group). Issues of less shore development (1\% of total) and less shore litter $(0.7 \%$ of total), the latter emphasizing spoil island clean-up, drew comparably from all access groups. The final two environmental subcategories, the removal of sunken boats and derelicts and the protection of grass flats, were of greatest concern to marina users and to ramp users respectively.

The professed need for more destinations and boating activity provisions, the $7^{\text {th }}$ ranked primary category, was disproportionate to reported corresponding detractors, with about four times the percentage contribution (8.4\% of total needs). The largest subcategory, "more 
waterfront restaurants," subsumed $2.5 \%$ of total responses, with an equally large percentage from within marina and dock users (4.1\% each), about four times that from within ramp users. More public, transient dockage to accommodate shore pursuits, ranking second in this category, also comprised $4.1 \%$ of all marina responses, with lesser input from dock and ramp users. Close behind was the call for more designated watersport areas, of which almost half (20 of 43 total responses) specified the need for water ski dedicated areas, often citing the loss of previous water ski areas to manatee zones. Dock users led with $2.7 \%$ of their total. Equal overall numbers wanted more beaches and parks or artificial reefs (each with $1.1 \%$ of total); the former was identified more by marina and dock users and the latter by marina and ramp users. Under beaches and parks, many $(\mathrm{n}=12)$ wanted more or better spoil island recreational provisions.

The last primary category with significant numbers (7.7\% of total) encompassed those answers addressing the need for boater education. All needs for heightened boater safety awareness, boat handling and etiquette training, and knowledge of regulations were assimilated in a single subcategory, encompassing almost $3 / 4$ of all category responses $(n=151)$ and figuring significantly across all access groups. The need for a mandatory boating course for all operators, often worded as a "mandatory safety course," was stressed by 51 respondents within this subcategory. The education primary category ranked $5^{\text {th }}$ for ramp users, as compared with $7^{\text {th }}$ for either marina or dock users, in part because of the user-group-specific call for training in ramp launch and retrieval. The call for environmental stewardship education ( $0.3 \%$ overall) was also associated with a higher percentage of ramp users (1\% of group).

Seventy respondents answered Question 25 with “no needs," making it the $8^{\text {th }}$ ranking primary category, with $2.6 \%$ of all responses, not stemming significantly more from any one access group. Only the need for less congested waterways had fewer mentions, with $1.4 \%$ of all responses and also ranking $9^{\text {th }}$ within all user groups.

The top ten needs subcategories overall are identified in Table 33. These accounted for 1,650 responses, or $61.5 \%$ of all analyzed responses to Question 25. Some conclusions:

- Three of the top ten subcategories fell under water access needs, including the leading subcategory of more ramps (14.1\% of total responses) and the $4^{\text {th }}$ ranked subcategory advocating more ramp parking (5.9\% of total). Each was significantly driven by the $1^{\text {st }}$ and $3^{\text {rd }}$ place ranking, respectively, within the large ramp access group.

- The second highest subcategory, calling for less manatee zone regulation, garnered $7.9 \%$ of all responses but a disproportionate $14.5 \%$ of all dock user responses. Similarly, the percentage of dock users calling for less speed zone regulation, the $6^{\text {th }}$ highest needs subcategory, was more than double that of the overall response percentage (11.7\% vs. 5.2\%).

- Ramp infrastructure improvements comprised the $3^{\text {rd }}$ highest subcategory with $7.2 \%$ of all responses (11.4\% of all ramp responses).

- The subcategory ranking $5^{\text {th }}$ overall, citing the need for boater education in all aspects of safety, etiquette, and regulatory measures, garnered $4.1 \%$ of marina, $5.0 \%$ of dock, and $6.3 \%$ of ramp responses. 
- Marinas, slips, and dry storage access needs comprised the $7^{\text {th }}$ highest subcategory, with $4.4 \%$ of all responses and a disproportionate $18.0 \%$ within the marina users.

- Infrastructure subcategories of dredging maintenance and better channel marks and signs occupied the $8^{\text {th }}$ and $9^{\text {th }}$ rankings, with $4.2 \%$ and $4.0 \%$ of total responses, respectively. Both of these needs ranked higher with marina and dock users than with ramp users, particularly with respect to dredging.

- Finally, the environmental need for better water quality completed the top ten subcategories, ranking $7^{\text {th }}$ within the dock access group and $8^{\text {th }}$ within marina and ramp user groups. 
Table 34. Primary Needs by Waterway Access Group.

\begin{tabular}{|c|c|c|c|c|c|c|c|c|c|}
\hline \multirow[b]{2}{*}{$\begin{array}{c}\text { BOATING NEEDS } \\
\text { CATEGORIES/SUBCATEGORIES }\end{array}$} & \multicolumn{3}{|c|}{ MARINA ACCESS } & \multicolumn{3}{|c|}{ DOCK ACCESS } & \multicolumn{3}{|c|}{ RAMP ACCESS } \\
\hline & Counts & $\begin{array}{c}\% \text { of all } \\
\text { Marina } \\
\text { Responses } \\
(n=412)\end{array}$ & $\begin{array}{c}\text { Marina } \\
\text { Category } \\
\text { Rank }\end{array}$ & Counts & $\begin{array}{c}\% \text { of all } \\
\text { Dock } \\
\text { Responses } \\
(n=753)\end{array}$ & $\begin{array}{c}\text { Dock } \\
\text { Category } \\
\text { Rank }\end{array}$ & Counts & $\begin{array}{c}\% \text { of all } \\
\text { Ramp } \\
\text { Responses } \\
(\mathbf{n}=1517)\end{array}$ & $\begin{array}{c}\text { Ramp } \\
\text { Category } \\
\text { Rank }\end{array}$ \\
\hline More Water Access & 119 & 28.9 & 1 & 91 & 12.1 & 3 & 486 & 32.0 & 1 \\
\hline For Boaters in General & 3 & 0.7 & & 1 & 0.1 & & 11 & 0.7 & \\
\hline Ramps & 18 & 4.4 & & 41 & 5.4 & & 318 & 21.0 & \\
\hline Ramp Parking & 4 & 1.0 & & 12 & 1.6 & & 141 & 9.3 & \\
\hline Marinas/Slips/Dry Storage & 74 & 18.0 & & 32 & 4.2 & & 13 & 0.9 & \\
\hline Anchorages and Public Moorings & 20 & 4.9 & & 5 & 0.7 & & 3 & 0.2 & \\
\hline Infrastructure Improvements & 92 & 22.3 & 2 & 132 & 17.5 & 2 & 249 & 16.4 & 2 \\
\hline Channel Marks/Waterway Signs & 27 & 6.5 & & 36 & 4.7 & & 42 & 2.7 & \\
\hline Dredging & 30 & 7.3 & & 58 & 7.7 & & 24 & 1.6 & \\
\hline Ramp Facilities & 11 & 2.7 & & 8 & 1.1 & & 173 & 11.4 & \\
\hline Full Service Marinas & 16 & 3.9 & & 8 & 1.1 & & 5 & 0.3 & \\
\hline Pumpout Stations & 3 & 0.7 & & 5 & 0.7 & & 1 & 0.1 & \\
\hline Fuel Docks & 2 & 0.5 & & 10 & 1.3 & & 3 & 0.2 & \\
\hline Bridges, Port Locks & 3 & 0.7 & & 7 & 0.9 & & 1 & 0.1 & \\
\hline Less Regulation & 46 & 11.2 & 4 & 243 & 32.3 & 1 & 165 & 10.9 & 4 \\
\hline Boating Regulation in General & 8 & 1.9 & & 8 & 1.1 & & 6 & 0.4 & \\
\hline Patrol Harassment & 1 & 0.2 & & 9 & 1.2 & & 3 & 0.2 & \\
\hline Speed Zones & 13 & 3.2 & & 88 & 11.7 & & 39 & 2.6 & \\
\hline No Wake Zones & 6 & 1.5 & & 23 & 3.1 & & 16 & 1.1 & \\
\hline Manatee Zones & 14 & 3.4 & & 109 & 14.5 & & 89 & 5.9 & \\
\hline Fishing Regulations & 2 & 0.5 & & 1 & 0.1 & & 8 & 0.5 & \\
\hline Canaveral Security & 2 & 0.5 & & 5 & 0.7 & & 4 & 0.3 & \\
\hline More Regulation/Enforcement & 31 & 7.5 & 6 & 65 & 8.6 & 6 & 195 & 12.9 & 3 \\
\hline Boating Regulations in General & 7 & 1.7 & & 12 & 1.6 & & 37 & 2.4 & \\
\hline Operator Licensure & 5 & 1.2 & & 11 & 1.5 & & 27 & 1.8 & \\
\hline PWC Regulation & 6 & 1.5 & & 9 & 1.2 & & 18 & 1.2 & \\
\hline Powerboat Regulation & 3 & 0.7 & & 2 & 0.3 & & 5 & 0.3 & \\
\hline Speed Zone Enforcement & 3 & 0.7 & & 13 & 1.7 & & 26 & 1.7 & \\
\hline More Pole/Troll Zones & 0 & 0.0 & & 0 & 0.0 & & 24 & 1.6 & \\
\hline More Patrol Presence on Waterways & 3 & 0.7 & & 8 & 1.1 & & 27 & 1.8 & \\
\hline Ramp Supervision & 0 & 0.0 & & 2 & 0.3 & & 15 & 1.0 & \\
\hline Drinking \& Boating & 1 & 0.2 & & 2 & 0.3 & & 4 & 0.3 & \\
\hline Recreational \& Comm. Fishing Regs & 1 & 0.2 & & 4 & 0.5 & & 11 & 0.7 & \\
\hline Crab Traps & 2 & 0.5 & & 2 & 0.3 & & 1 & 0.1 & \\
\hline Environmental Protection & 36 & 8.7 & 5 & 66 & 8.8 & 5 & 126 & 8.3 & 6 \\
\hline Improved Water Quality & 14 & 3.4 & & 34 & 4.5 & & 37 & 2.4 & \\
\hline Less Island/Shore Trash & 2 & 0.5 & & 7 & 0.9 & & 10 & 0.7 & \\
\hline Sunken Boats, Derelicts Removal & 8 & 1.9 & & 4 & 0.5 & & 8 & 0.5 & \\
\hline More Fish & 4 & 1.0 & & 10 & 1.3 & & 48 & 3.2 & \\
\hline Less Shore Development/More Nat. Areas & 8 & 1.9 & & 8 & 1.1 & & 11 & 0.7 & \\
\hline Grass Flats Protection & 0 & 0.0 & & 3 & 0.4 & & 12 & 0.8 & \\
\hline More Destinations/Activity Provisions & 53 & 12.9 & 3 & 86 & 11.4 & 4 & 87 & 5.7 & 7 \\
\hline Public Places to Visit & 5 & 1.2 & & 6 & 0.8 & & 2 & 0.1 & \\
\hline Waterside Restaurants & 17 & 4.1 & & 31 & 4.1 & & 18 & 1.2 & \\
\hline Public and Transient Dockage & 17 & 4.1 & & 14 & 1.9 & & 15 & 1.0 & \\
\hline Beaches, Parks & 6 & 1.5 & & 11 & 1.5 & & 12 & 0.8 & \\
\hline Designated Watersport Areas & 2 & 0.5 & & 20 & 2.7 & & 21 & 1.4 & \\
\hline Artificial Reefs & 6 & 1.5 & & 4 & 0.5 & & 19 & 1.3 & \\
\hline Boater Education & 19 & 4.6 & 7 & 46 & 6.1 & 7 & 142 & 9.4 & 5 \\
\hline Safety/Etiquette/Boating Skills/Regs & 17 & 4.1 & & 38 & 5.0 & & 96 & 6.3 & \\
\hline Environmental Stewardship & 2 & 0.5 & & 3 & 0.4 & & 15 & 1.0 & \\
\hline PWC Education & 0 & 0.0 & & 4 & 0.5 & & 5 & 0.3 & \\
\hline Ramp Launch \& Retrieval & 0 & 0.0 & & 1 & 0.1 & & 26 & 1.7 & \\
\hline Less Congested Waterways & 3 & 0.7 & 9 & 3 & 0.4 & 9 & 31 & 2.0 & 9 \\
\hline Fewer Boaters & 3 & 0.7 & & 3 & 0.4 & & 31 & 2.0 & \\
\hline No Needs & 13 & 3.2 & 8 & 21 & 2.8 & 8 & 36 & 2.4 & 8 \\
\hline
\end{tabular}




\section{Part 3 - Spatial Analysis of Boating Patterns}

\section{Chapter 7. Mapping Marina and Ramp Patronage}

An important element of the Brevard County recreational boating characterization was to determine the general land-side service areas for the County's waterway access facilities (i.e., marinas and boat ramps). This analysis relied, first, upon identifying marina and ramp patrons and, second, mapping where those patrons live relative to the facilities that they used. To accomplish this, Florida Sea Grant personnel collected vessel registration numbers from boats berthed in Brevard County marinas. A total of 34 marinas were visited, of which 28 agreed to participate in the study. Twenty-two of the 28 participating marinas granted access to their docks, dry storage racks, or client mailing lists. In addition, Brevard County Parks and Recreation staff and Florida Sea Grant personnel collected automobile and vessel trailer registration numbers at 30 boat ramps on 54 selected weekend days and week days over a one year period (March 2006 through February 2007). Vessel, trailer, and automobile tag numbers collected at marinas and ramps were compared to registration data maintained by the Florida Department of Highway Safety and Motor Vehicles to obtain names and mailing addresses for marina and ramp patrons. Mailing addresses were, in turn, mapped using United States Postal Service (USPS) address locating or "geocoding" software, ${ }^{1}$ as presented in Figure 1.

\section{Marina and Ramp Use Landside Profile}

The survey of vessels kept in Brevard County marinas yielded 1,146 patron names and mailing addresses from an estimated 2,327 wet slip and dry rack spaces. This sample thereby accounted for approximately $50 \%$ of all marina users, assuming a $100 \%$ occupancy rate. A total of 74 addresses were mapped to out-of-state locations; 1,072 were located within Florida. Of the marina patrons who had a Florida address, 66.2\% $(\mathrm{n}=710)$ were located within Brevard County (Figure 2). Orange (12.9\%), Seminole (7.1\%), Indian River (3.6\%), and Volusia (2.2\%) counties accounted for an additional $25.8 \%$ of Brevard County marina patronage. Together, the top five counties accounted for $92 \%$ of Brevard County marina patronage.

Vessel trailers and tow vehicles were inventoried at Brevard County ramps on 54 sample days, throughout a one year period, yielding 22,018 log sheet entries (trailer and/or tow vehicle tag). A total of 9,025 Florida tag numbers were linked to unique addresses, which could be mapped using USPS geocoding software. Of these, 8,966 addresses were in Florida. Of the ramp patrons who had Florida addresses, 46.3\% were located within Brevard County (Figure 3). Orange (14.5\%), Seminole (9.2\%), Indian River (7.5\%), and Volusia (5.2\%) counties contributed an additional $36.4 \%$ to Brevard County ramp use. Together, the top five counties accounted for almost $83 \%$ of Brevard County ramp patronage.

\footnotetext{
${ }^{1}$ The term geocoding refers to the process of locating addresses to street segments. This was accomplished by using United States Postal Service ZIP-code matching software. Approximately, ninety-eight percent of the addresses for marina and ramp users were matched to the ZIP + 4 (nine-digit zip code) which results in a locational error that is generally no greater than four houses along a street segment.
} 
Geocoded ramp patron data can also be used to map service areas for individual boat ramps. Figure 4 illustrates the land-side service areas (that capture $81 \%$ of the ramp patrons) for the popular Freddie Patrick Park ramp at Port Canaveral. The service areas were identified using a GIS method that incorporated criteria established by Applebaum (1966) for determining a retail market share boundary based on consumer travel distances. Applebaum suggested that the boundaries of a primary service area encompass a geographic area that accounts for between $70-$ 80 percent of the users or consumers within that market. Mapped patronage data can be used to estimate demand for particular boat ramps based on use profiles (obtained from this study) and the number of registered and trailerable boats that are located within delineated service areas.

\section{Florida Distribution of Brevard County Ramp and Marina Patrons}
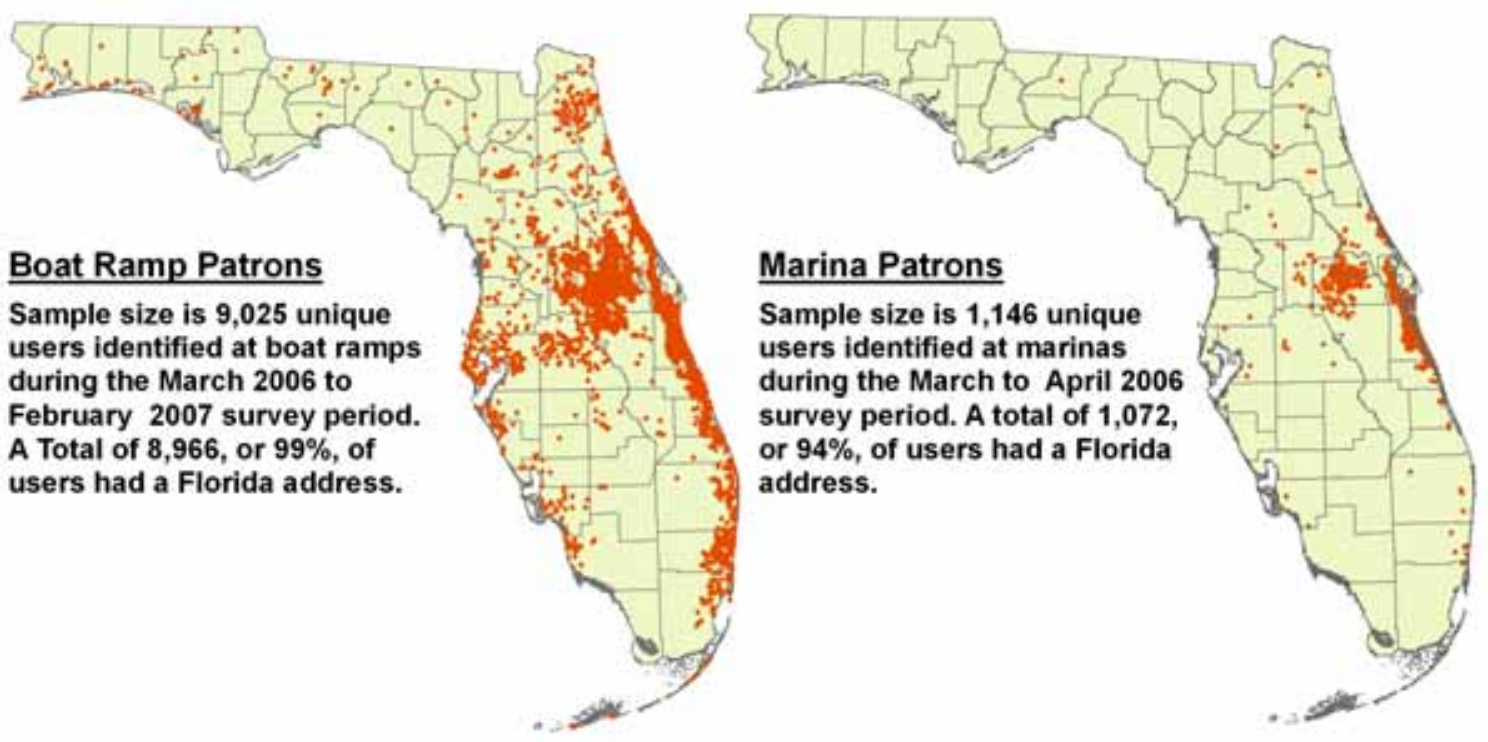

Vessel trailer and tow vehicle tag numbers were identified at Brevard County boat ramps. Vessel registration numbers were also identified from boats berthed or kept at Brevard County marinas and dry-storage facilities. This information was compared with registration data to provide names and mailing addresses for ramp and marina patrons. Mailing addresses were mapped using U.S. Postal Service address locating software.

Figure 1. Florida Distribution of Brevard County Ramp and Marina Patrons. 


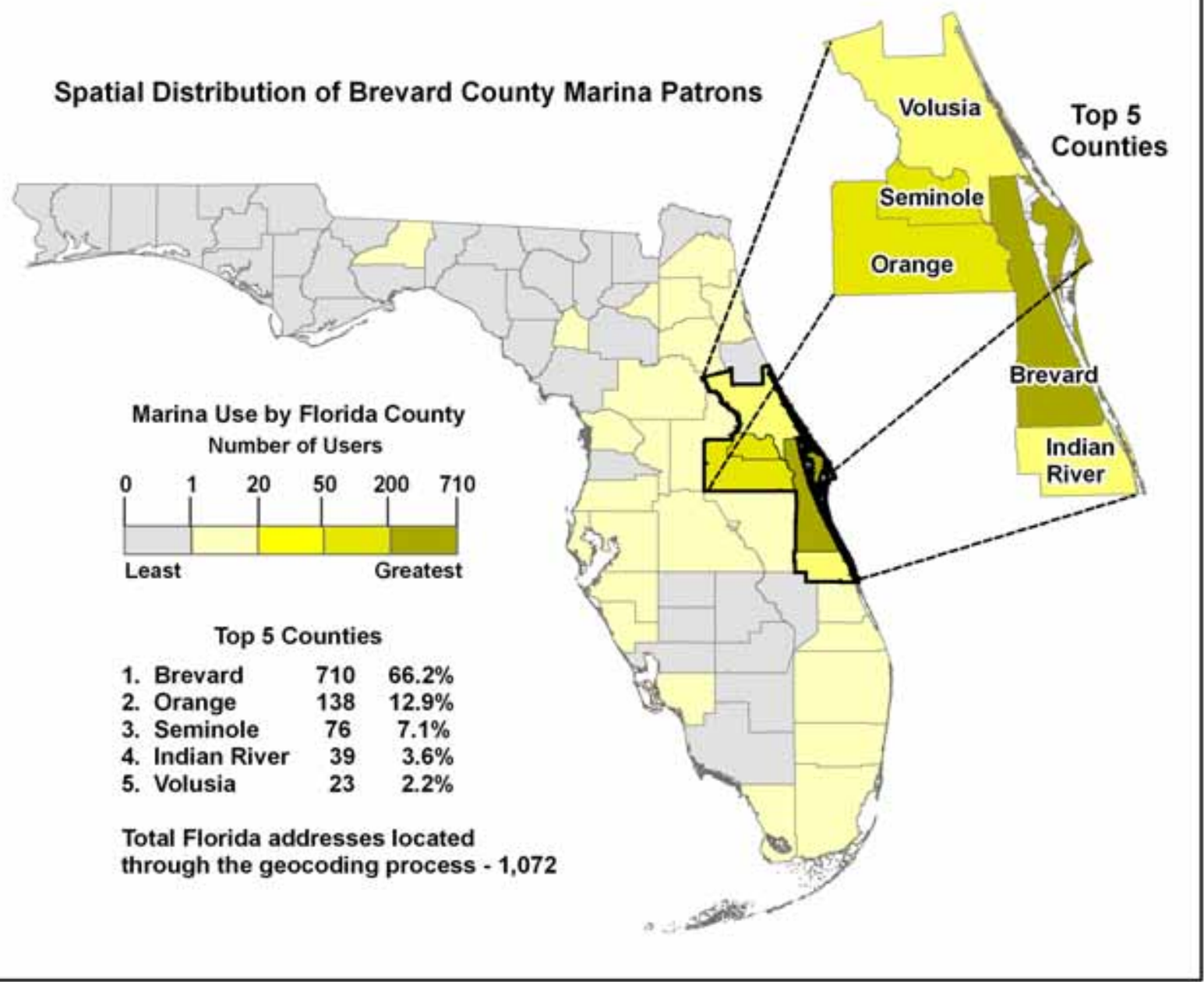

Figure 2. Florida Distribution of Brevard County Marina Patrons. 


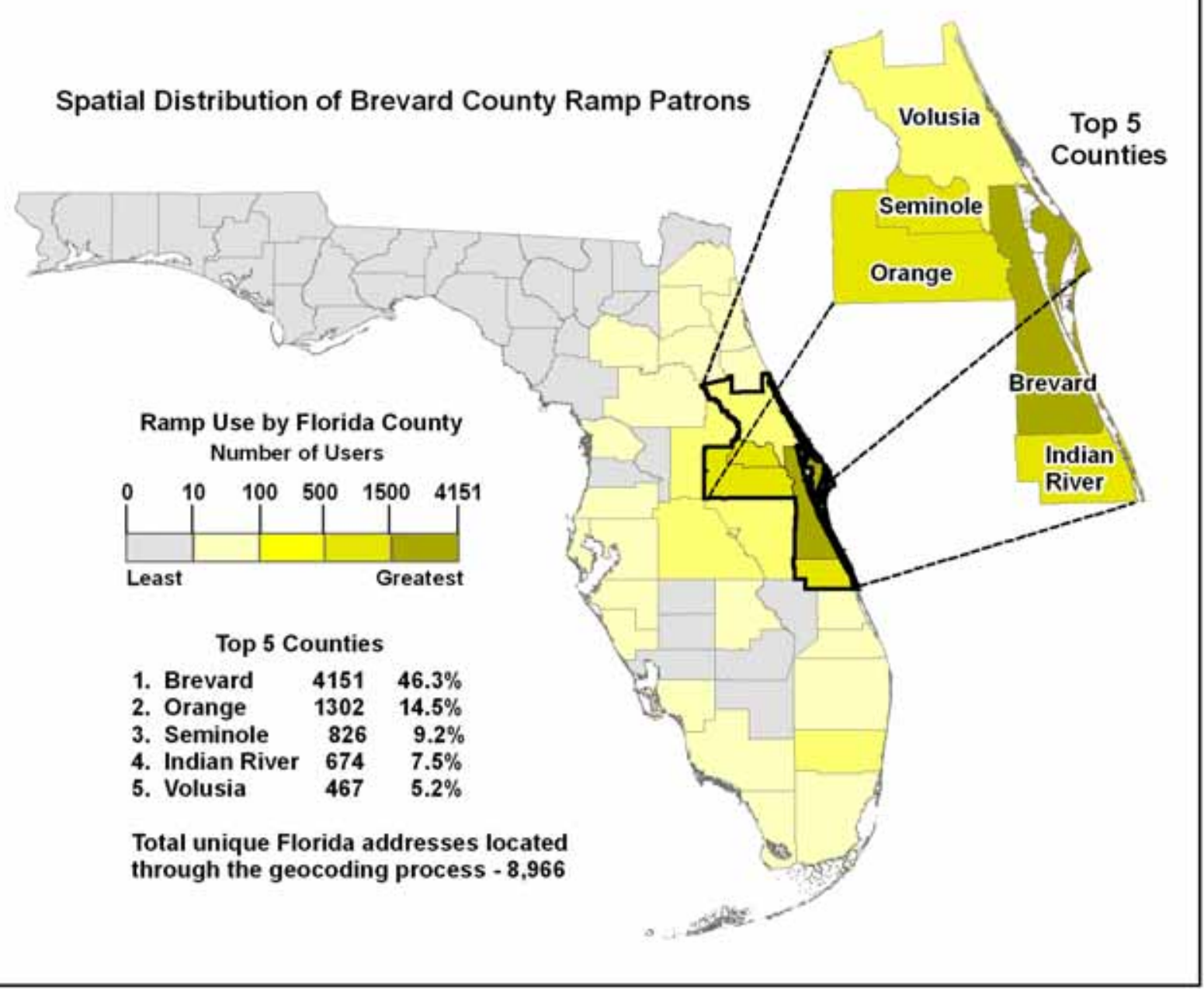

Figure 3. Florida Distribution of Brevard County Ramp Patrons. 


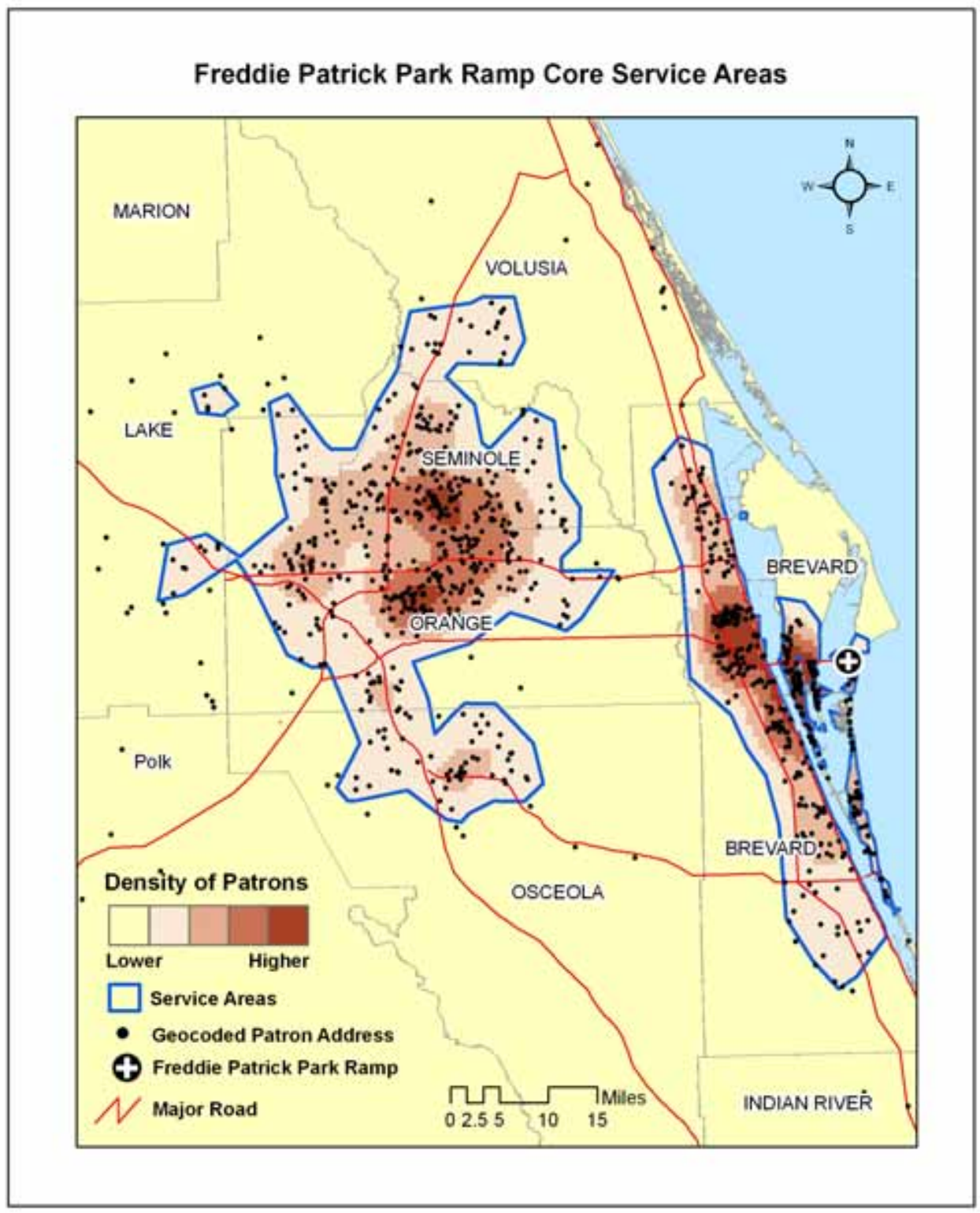

Figure 4. Core Service Areas for the Freddie Patrick Park Boat Ramp.

\section{Ramp Use vs. Parking Capacity}

The parking capacities of boat ramps were estimated and compared with the average numbers of boat trailers observed during peak (May, June, and July), shoulder (March, April, August, September, and October), and off-peak (November, December, January, and February) use periods. ${ }^{2}$ The analysis was limited to the 42 weekend days that ramp visits took place (Table

\footnotetext{
${ }^{2}$ Use periods were identified by a cluster analysis of the number of days each month that survey respondents reported taking boat trips. See Chapter 5 for a more detailed description of the seasonal analysis.
} 
1). The results indicate that a number of boat ramps (e.g., Bairs Cove, Freddie Patrick, H. Pollak, John Jorgensen, Kelly Park, Kennedy Park, Mims, Parrish Park, and Sebastian Inlet State Park, Sebastian Yacht Club, Main Street) are operating close to or above estimated parking capacity during some use periods By contrast, some ramps may be underutilized relative to estimated parking capacities (e.g., Alex Goode, Ballard Park, Bio-Lab, Constitution Bi-Centennial, Front Street, Eau Gallie, Lee Wenner, Long Point, Old Beacon 42, and Ramp Road).

Table 1. Ramp Parking Capacity and Utilization Estimates.

\begin{tabular}{|l|c|c|c|c|}
\hline \multirow{2}{*}{ Ramp Name } & \multirow{2}{*}{$\begin{array}{c}\text { Parking } \\
\text { Spaces }\end{array}$} & \multicolumn{3}{c|}{$\begin{array}{c}\text { *Average Number of Trailers } \\
\text { Observed for Time Periods }\end{array}$} \\
\cline { 3 - 5 } & & Peak Use & Shoulder & Off-Peak \\
\hline 1st Street & 10 & 4 & 2 & 2 \\
\hline Alex Goode & 28 & 8 & 6 & 4 \\
\hline Bairs Cove & 40 & 27 & 38 & 35 \\
\hline Ballard Park & 50 & 29 & 28 & 13 \\
\hline Bio-Lab & 30 & 10 & 10 & 9 \\
\hline Constitution Bicentennial & 30 & 9 & 9 & 7 \\
\hline Eau Gaille & 40 & 17 & 17 & 11 \\
\hline Eddy Creek & 12 & 2 & 6 & 5 \\
\hline Freddie Patrick & 100 & 91 & 64 & 34 \\
\hline Front Street & 40 & 14 & 13 & 6 \\
\hline H Pollak & 7 & 12 & 8 & 4 \\
\hline John Jorgensen & 20 & 33 & 30 & 23 \\
\hline Kelly Park & 37 & 39 & 27 & 24 \\
\hline Kennedy Point & 28 & 24 & 24 & 13 \\
\hline Kiwanis Island & 15 & 9 & 8 & 8 \\
\hline Lee Wenner & 43 & 22 & 18 & 3 \\
\hline Long Point & 25 & 4 & 4 & 2 \\
\hline Main Street Boat Dock & 25 & 22 & 19 & 13 \\
\hline Marina Point & 30 & 20 & 15 & 9 \\
\hline Mims & 8 & 10 & 6 & 5 \\
\hline Old Beacon 42 & 50 & 23 & 27 & 22 \\
\hline Old Scottsmoor & 16 & 9 & 8 & 8 \\
\hline Parrish Park & 45 & 47 & 63 & 33 \\
\hline Pineda Landing (POW/MIA) & 40 & 20 & 18 & 5 \\
\hline Port End Park & 28 & 15 & 16 & 8 \\
\hline Port St. John & 30 & 22 & 20 & 10 \\
\hline Ramp Road & 20 & 6 & 7 & 12 \\
\hline Sebastian Inlet St. Park & 30 & 34 & 34 & 26 \\
\hline Sebastian Yacht Club & 40 & 53 & 49 & 31 \\
\hline Wabasso Causeway Park & 35 & 23 & 24 & 19 \\
\hline
\end{tabular}

*Peak Use Period (May, June, July)

Shoulder Use Period (March, April, August, September, October)

Off-Peak Use Period (November, December, January, February) 


\section{Chapter 8. Mapping Boating Patterns}

\section{General Clustering Patterns}

This chapter presents the results of a GIS analysis that mapped the distribution or spread of the digitized trip information as "density of occurrence." Continuous density surfaces generated by the GIS illustrate the degree of concentration or clustering of digitized trip information. General clustering patterns for travel routes, destinations, and congested areas were mapped and described using 100 meter grid cells and a search radius of 1,000 meters.

Route densities are depicted in Figure 5. The greatest density of vessel traffic is restricted to Intracoastal Waterway segments within the Indian River Lagoon-from the Haulover Canal in the north to the Sebastian Inlet to the south. Vessel traffic density is also great within the lower reaches of the Banana River to Port Canaveral. Beyond the barrier islands, the flow of boat traffic is generally dispersed though evidence of some clustering of routes exists toward popular artificial and natural reefs in the Atlantic.

Figure 6 displays favorite destinations identifying the locales where respondents most like to visit on a typical recreational boating outing. The density analysis revealed several prime boating destinations: The Sebastian Inlet area, a number of small spoil islands along the ICW south of the Melbourne Causeway, "Ski Island" at the entrance to the Port Canaveral lock, two islands within the Banana River near the lower reaches of Newfound Harbor ("Bird Island" and "Honeymoon Island"), and at spoil islands north of the Brewer Memorial Parkway. Prominent Causeways including Bennett, Merritt Island, Pineda, and Eau Gallie also represent favorite boating locales, some of which are associated with a boat ramp. Some areas within the Mosquito Lagoon were also identified as being popular boating destinations.

Figure 7 illustrates areas where boaters experience congestion defined in Question 18 as "more boats than you prefer." The analysis shows that respondents experience the most congestion at favorite boating destinations including the Sebastian Inlet, "Ski Island", near two small islands at the confluence of the Banana River and Newfound Harbor ("Bird Island" and "Honeymoon Island"), the narrow confluence of the Banana River and the Indian River Lagoon near Dragon Point and the Eau Gallie Causeway, and at the Haulover Canal. An area near Vanns and Bird Islands in the Mosquito Lagoon was also associated with congestion. In addition, a number of causeways were identified as congested by survey respondents - particularly those that contained a boat ramp (e.g., Merritt Island, Pineda, Eau Gallie, Max Brewer Memorial Parkway).

Normal color Digital Ortho Photo Quadrangle imagery with one-meter resolution was used for the digitization of trip information reported on 1:160,000 maps by survey respondents. As a result, information collected as part of the study can also be mapped at higher resolutions than shown in Figures 5 through 7. This is illustrated by Figure 8 which shows a close-up of favorite destination spots mapped by survey respondents and a density analysis of those points for the Merritt Island area. Figure 8 was generated using the density kernel function with a search radius of 500 meters and a mapping resolution of 100 meters. 


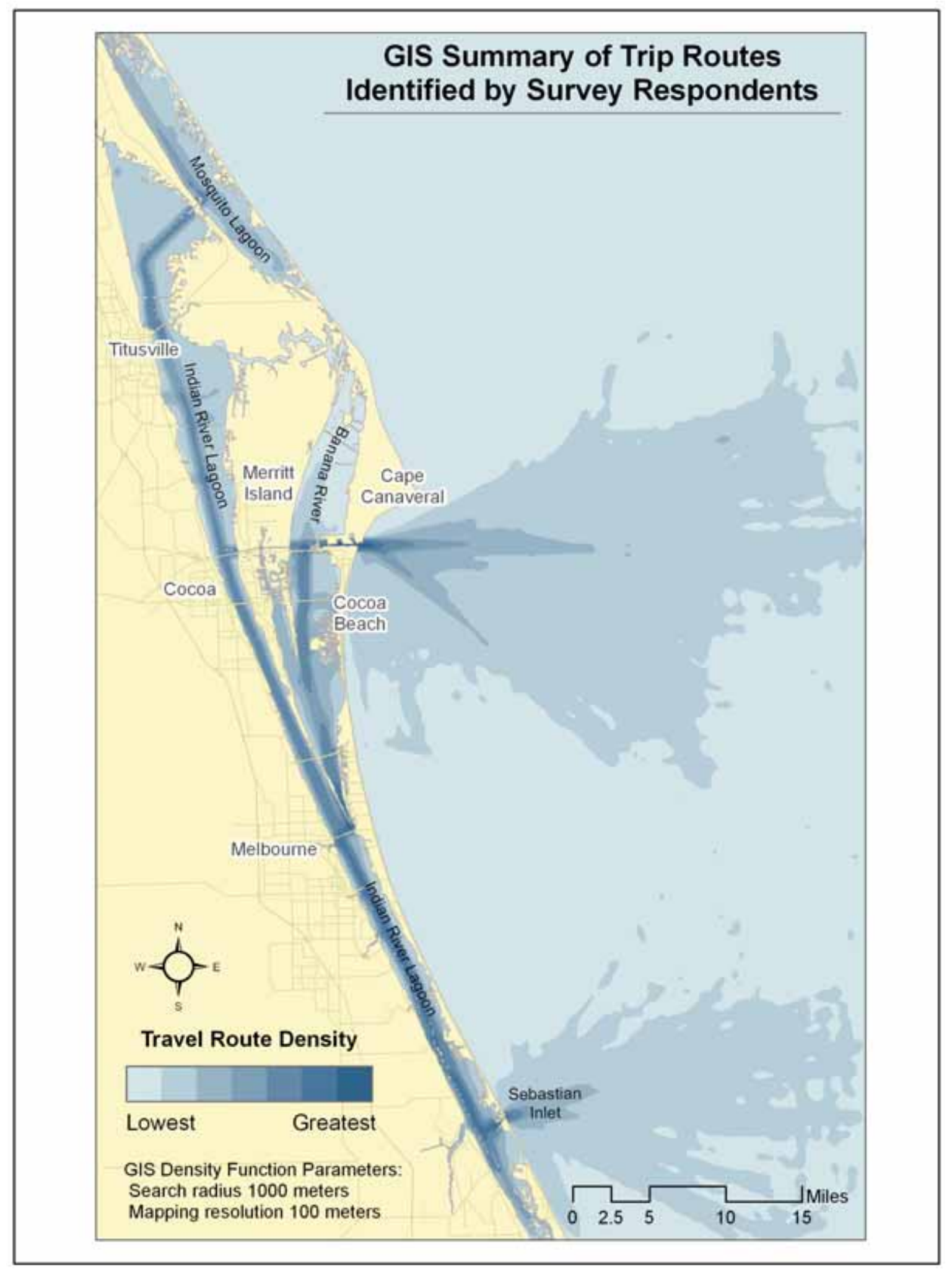

Figure 5. Travel Corridors as Summarized with the GIS. 


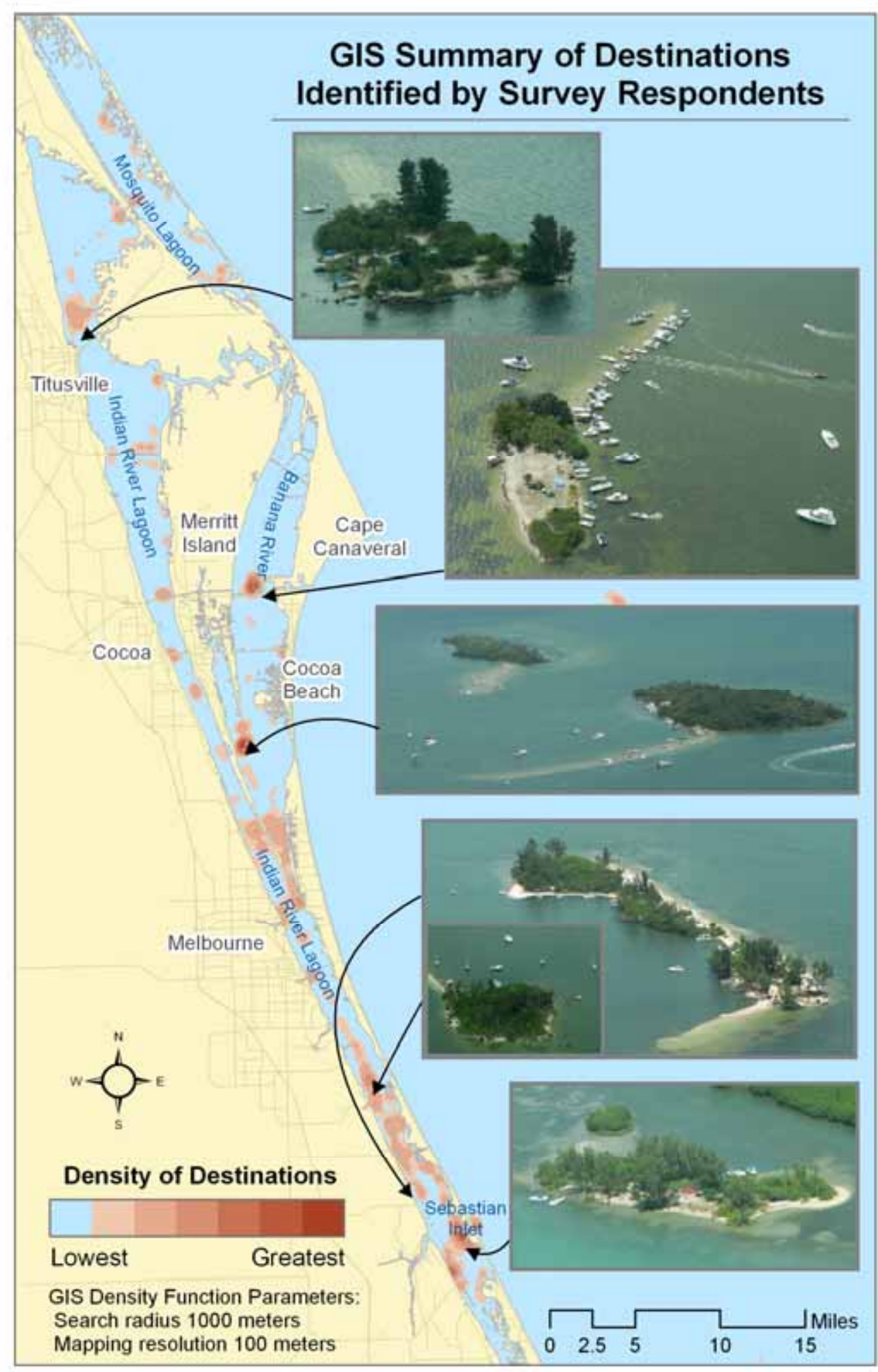

Figure 6. Favorite Destinations as Summarized with the GIS. 


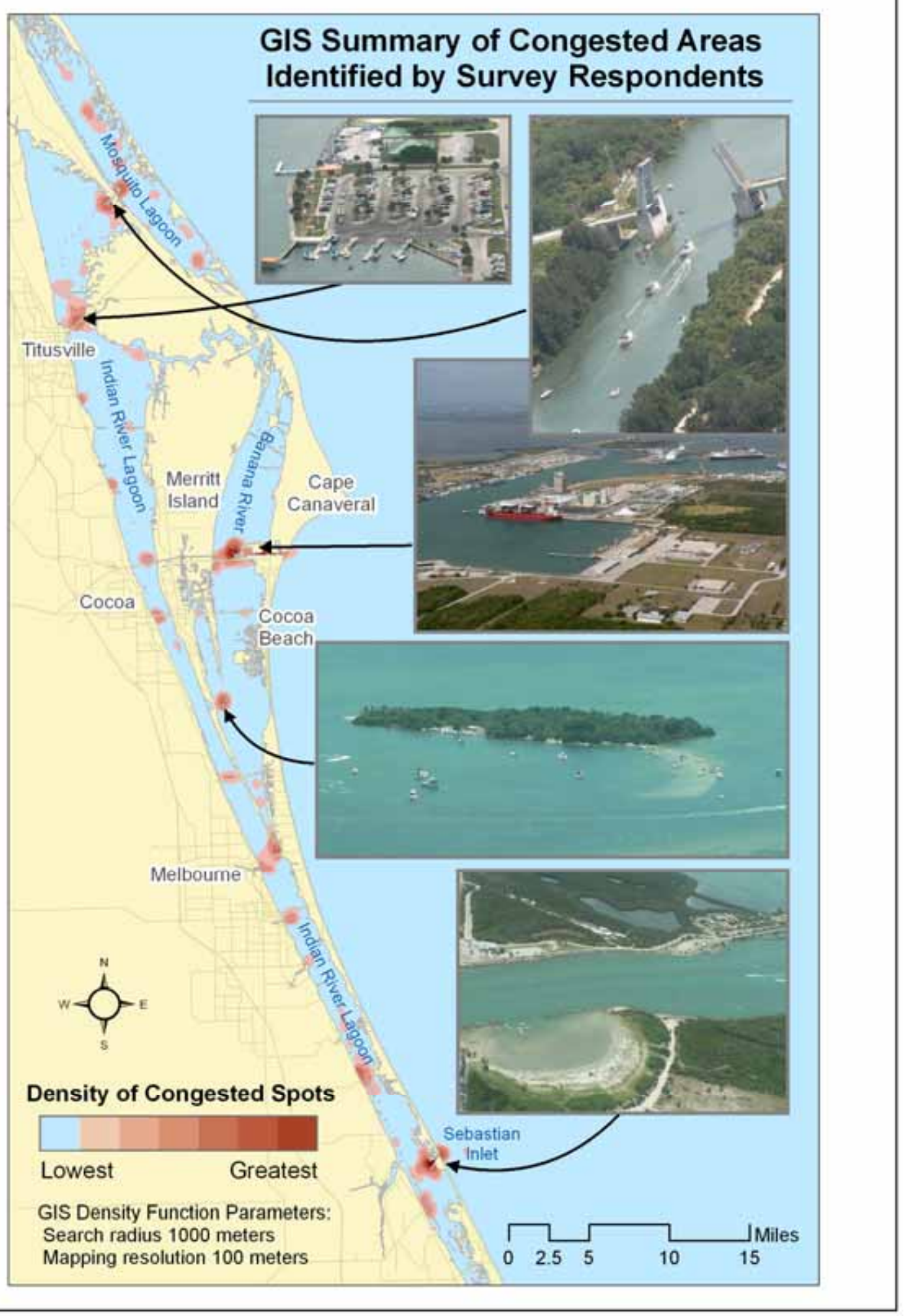

Figure 7. Congested Areas as Summarized with the GIS. 


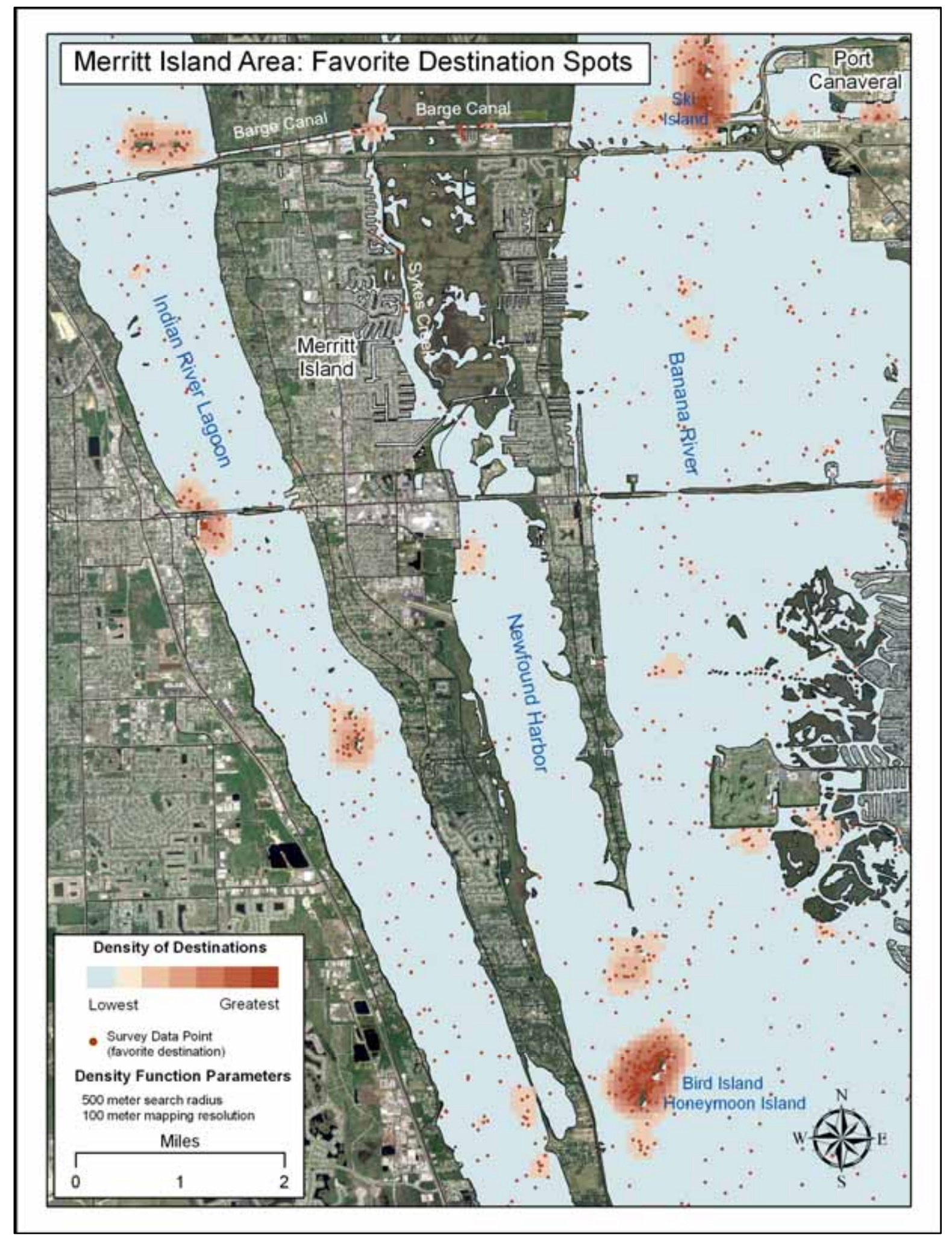

Figure 8. Close-up of Favorite Destination Spots for the Merritt Island Area. 


\section{Seasonal Boating Patterns}

Analysis of mapped destination points reported by survey respondents identified areas where clustering occurred for each of the three seasons. The Getis-Ord G statistic (Getis \& Ord, 1996) was calculated and the Z-scores of the statistic ( $\left.\mathrm{Gi}^{*}\right)$ were rendered using ArcMap to reveal hot-spots of boating destinations.

A cluster analysis of the reported number of boating trips taken each month determined that boating destinations could be statistically grouped into a "peak," an "off-peak," and a "shoulder" season (see Part 2 for the complete seasonal analysis and identification of seasonal clusters). The peak season of boating activity was late spring and early summer, in May, June and July. The off-peak season was in winter, from November through February. The two shoulder seasons included March and April in the spring and August through October in the late summer to early fall. The boating destinations, after being digitized into a GIS, were separated into peak, offpeak and shoulder seasons (using the month that the trip occurred as the identifier), with the two shoulder seasons aggregated into one group.

A one kilometer-square grid was created for the extent of the study area, and the number of reported destination points was counted for each grid cell. This count gave a weighted measure of the number of boating destinations per cell. The weighting was used to calculate the Getis-Ord $\mathrm{G}$ statistic for the center point of that cell.

The Getis-Ord G-statistic gives a measure of clustering relative to a neighborhood of values. So, if features that have high values are clustered in one area, the G-statistic will be larger than would be expected if the values were the result of random chance, and that feature is part of a "hot spot." For this analysis, the G statistic was calculated using a fairly restricted neighborhood of 1000 meters. This means that only the cells immediately adjacent to the sides of any given cell were considered in the neighborhood calculation. From these values, $Z$ scores were calculated and those with statistically significant scores were mapped. For the analysis, any $Z$ scores greater than $1.96(\alpha=0.5)$, were shown (see Figures 9,10 and 11$)$. The results were mapped excluding any $Z$ scores less than or equal to 1.96. To render the results, a Jenks natural breaks classification method was used to summarize the results into seven classes, which range nominally from the lowest levels of clustering to the highest levels of clustering. Highest levels are shown on the maps in the saturated red color, while lowest levels are shown in a light pink. Intermediate values are color ramped in between.

The maps show that there are some areas in the waters of Brevard County that have consistent heavy clustering of recreational boating destinations throughout all three seasons. Prominent among these year-round hot spots are the Sebastian Inlet area and some small islands in the Banana River. The results also showed seasonal variations in use for some areas. In peak season, the Mosquito Lagoon is clearly a boating destination hot spot, less so during transition periods, and not at all during the off-peak winter months. In addition, some areas off-shore see increased use and could be considered boating destination hot spots, probably corresponding with off-shore fishing areas near artificial and natural reefs. 
The same analytic process was followed for the off-peak destinations but with a 500 meter grid with a $1 \mathrm{~km}$ neighborhood. Although the results showed a better resolution, the time it took to run the analysis (approximately 14 hours) outweighed the benefits of the finer resolution at the reporting scale. To better understand the dynamics of the bay areas between the barrier islands and the mainland, it would be worthwhile to carry out the same analysis on a finer spatial and temporal scale, excluding areas of land and open water that might skew the results. 


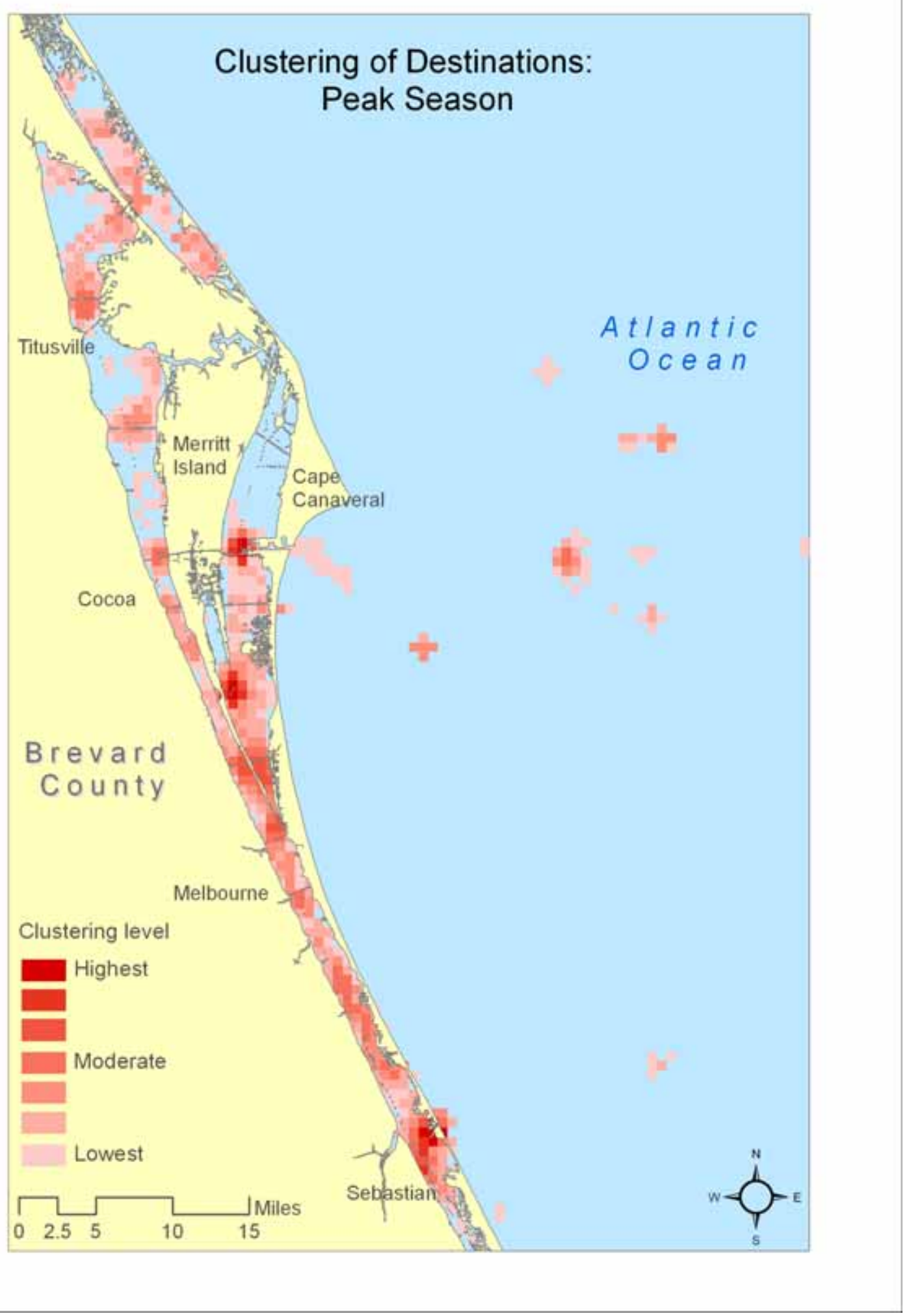

Figure 9. Destination Clustering for the "Peak" Boating Season. 


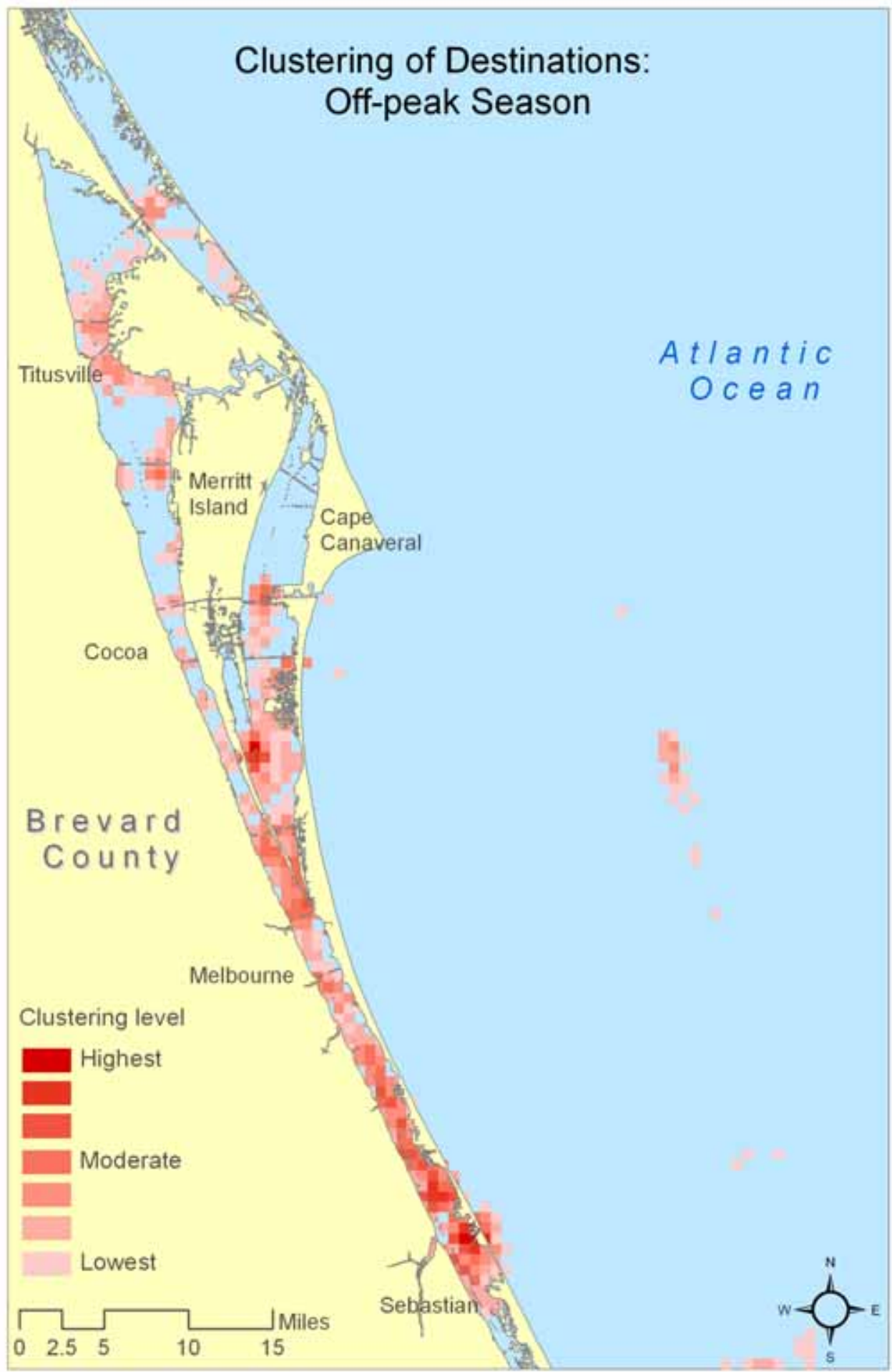

Figure 10. Destination Clustering for the "Off-Peak" Season. 


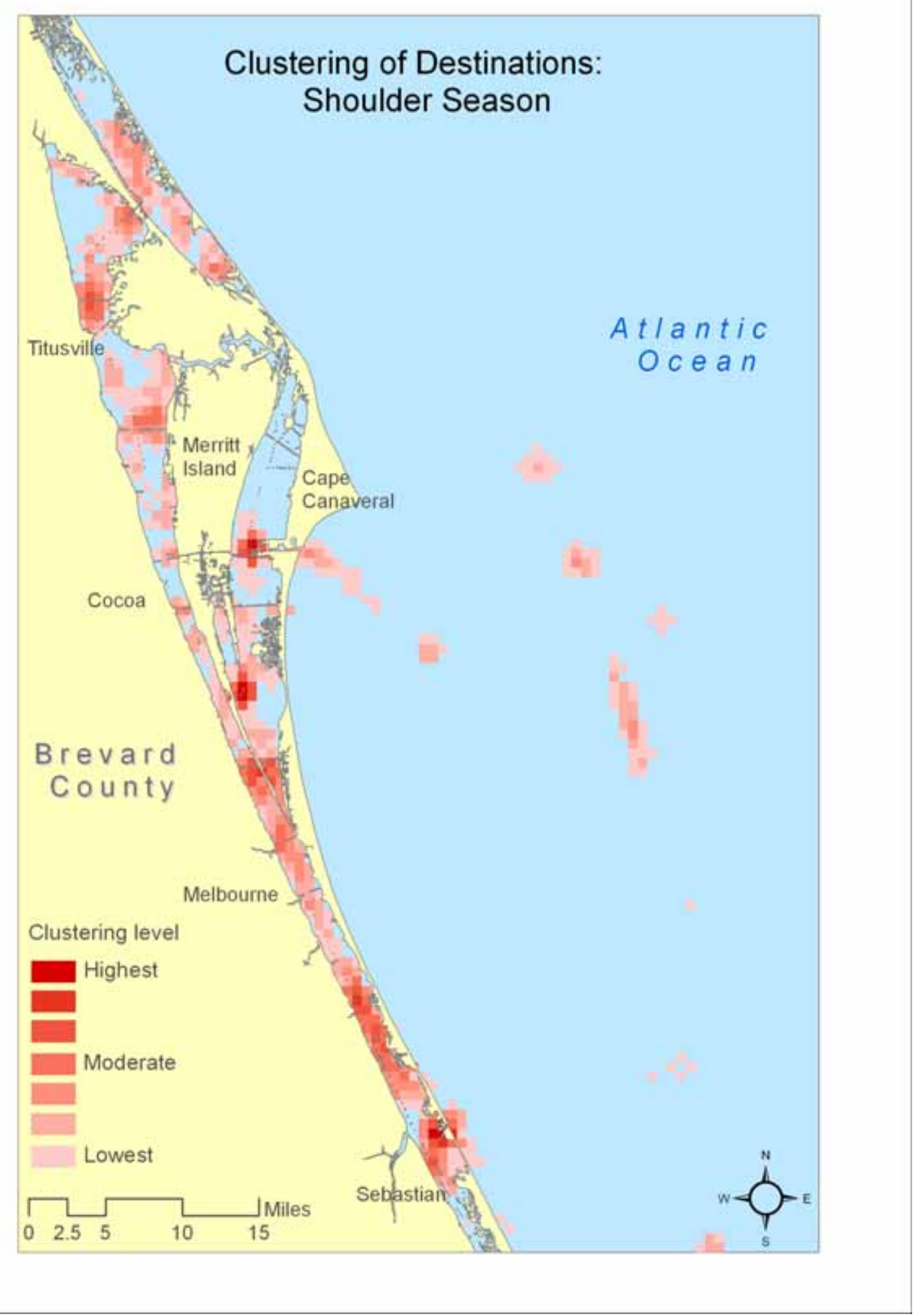

Figure 11. Destination Clustering for the "Shoulder" Season. 


\section{Chapter 9. Summary and Conclusions}

The purpose of this study was (1) to quantify and map public access facility use through an inventory of marinas and boat ramps, and (2) to characterize the use patterns of boaters on coastal waterways within and around Brevard County by season and by waterway access type. The analysis presented in this report was based upon information collected during visits to marinas and boat ramps and through the distribution of three waves of mail surveys that targeted boaters who accessed the water from marina wet slips, marina dry-storage facilities, public ramps, and private docks. A compilation of the responses to a subset of survey questions reveals that a typical respondent to the survey can be described as follows:

- Is a year-round Florida resident and is approximately 54 years of age;

- Has, on average, 20 years of boating experience on Florida waterways and has taken a boating safety or seamanship course;

- Owns an open fishing vessel about 22 feet in length;

- Takes an average of two to three trips per month (primarily on weekends), with more trips taken during the late spring and summer months (May through July) and fewer trips during winter months (December through February);

- Begins a trip at approximately 8AM and spends about $5 \frac{1}{2}-6$ hours on the water;

- Shows a preference for the following water-based activities in order of importance: fishing, nature viewing, and sightseeing;

- Perceives that a lack of seamanship and courtesy in other boaters, excessive regulation (primarily manatee zones), and/or the lack of water access (principally ramps and ramp parking) detract most from recreational boating enjoyment, and, lastly;

- Believes that better water access (principally more ramps and parking) and infrastructure improvements (chiefly improved channel dredging and marking) and/or fewer regulations (primarily manatee zones) would do most to improve recreational boating enjoyment.

The analysis first relied on the identification of primary boating periods by use of a cluster analysis based on the reported number of days per month that respondents spent boating. The cluster analysis revealed the presence of three distinct boating periods: a peak season (May, June, July), an off-peak season (November, December, January, and February), and a shoulder season (March, April, August, September, and October) - a classification that did not conform to the popular convention of "spring", "summer", "fall" and "winter." These boating periods differed from those determined for Southwest Florida (Greater Charlotte Harbor), highlighting regional differences in boating use in a state known for its "year-round boating season" (Sidman, et al. 2005). 
The second analytical element involved the evaluation of seasonal trends among the four waterway access user groups. The analysis highlighted trends in (a) trip departure time, (b) trip duration, (c) weekend vs. weekday use patterns, and (d) boating activities. The highly skewed distributions of reported trips taken during the three boating periods reflect the fact that two general boating populations are represented: (a) typical users - those that conform to period and yearly trip averages; and (b) power users - those that boat more frequently than a typical user. It is likely that the mail survey method captured the use profiles of boaters that represent both average and above-average users. Boaters characterized as infrequent users may not be adequately captured. In spite of potential for non-response bias, an argument can be made that the survey data and results reflect boaters who more frequently use the resource.

The third analytical component of this study focused on (1) the spatial distribution of ramp and marina patrons, and (2) spatial patterns of waterway use and period-specific boating patterns from reported trip data captured by the three mail survey waves. The Getis \& Ord G*-statistic (a measure of localized spatial dependence) was used to map and evaluate favorite boating destinations identified by mail survey respondents. A visual inspection of the resulting maps show that some boating "hot-spots" are popular throughout the year, while others (e.g., Mosquito Lagoon, and artificial and natural reefs in the Atlantic) experience distinct seasonal differences in use intensity.

The results underscore the importance of collecting boating data throughout the course of a year via multiple contacts (i.e., survey waves that allow for the collection of data during different boating seasons). Of equal importance, the analysis supports the targeting of the four waterway access groups - user groups that show statistically significant variability in trip behavior, trip characteristics, and use patterns over boating seasons. 


\section{Literature Cited}

Applebaum, W. 1966. "Methods for Determining Store Trade Areas, Market Penetration and Potential Sales," Journal of Marketing Research, 3: 127-141.

Antonini, G., Fann, D., and Roat, P. 1999. A Historical Geography of Southwest Florida Waterways Volume One: Anna Maria Sound to Lemon Bay. Florida Sea Grant Publication SGEB47. University of Florida, Gainesville, Florida.

Antonini, G., West, N., Sidman, C., Swett, R. 2000. A Recreational Boater-Based Method for Redesigning the NOS Small Craft Chart. Florida Sea Grant Publication TP-107. University of Florida, Gainesville, Florida.

Antonini, G., Zobler, L., Sheftall, W., Stevely, J., Sidman, C. 1994. Feasibility of a NonRegulatory Approach to Bay Water Anchorage Management for Sustainable Recreational Use. Florida Sea Grant Publication TP-74. University of Florida, Gainesville, Florida.

Falk, J., Graefe, A., Drogin, E., Confer, J., Chandler, L. 1992. Recreational Boating on Deleware's Inland Bays: Implications for Social and Environmental Carrying Capacity. Deleware Sea Grant Publication DEL-SG-19-92. University of Deleware, Newark.

Getis A, and Ord J., K. 1996. “Local Spatial Statistics: An Overview.” In Longley P, Batty M (eds) Spatial Analysis Modeling in a GIS Environment. Cambridge, GeoInformation International 269-85.

Leeworthy, V., and Wiley, P. 2001. National Survey on Marine Recreation and the Environment 2000: Current Participation Patterns in Marine Recreation. A Report to the U.S. Department of Congress National Oceanographic Atmospheric Administration. Silver Springs Maryland.

Letson, D. 2002. "Economic Value and Environmental Quality: Florida's Coastal Resources, in Marine Recreational Fishing" in Florida Coastal Environmental Resources: A Guide to Economic Valuation and Impact Analysis. Letson D. \& Milon, J.W., eds. Florida Sea Grant Report, SGR 124. University of Florida, Gainesville.

McCall, H. 1982. Sampling and Statistics Handbook for Research. Iowa State University Press. Ames, Iowa.

National Marine Manufacturers Association. 2005. 2004 U.S. Recreational Boat Registration Statistics. Available on-line at http://www.nmma.org/facts/local/documents/2004RegistrationsSummary.pdf

Sidman. C., and Flamm, R. 2001. A Survey of Methods for Characterizing Recreational Boating in Charlotte Harbor, Florida. Florida Sea Grant Publication TP-109. University of Florida, Gainesville, Florida. 
Sidman, C, Fik, T., and Sargent, W. 2004. A Recreational Boating Characterization for Tampa and Sarasota Bays. Florida Sea Grant Publication TP-130. University of Florida, Gainesville, Florida.

Sidman, C., Fik, T., Swett, R., Sargent, B. and Fann, S. 2005. Estimating Land and Water-Side Service Areas and Use Potential for Boat Ramps: A Case Study of Tampa and Sarasota Bays. Florida Sea Grant Publication TP-142. University of Florida, Gainesville, Florida.

Sidman, C., Swett, R., Fik, T., Fann, S., Fann, D. and Sargent, B. 2005. A Recreational Boating Characterization for the Greater Charlotte Harbor. Florida Sea Grant Publication TP-150. University of Florida, Gainesville, Florida.

Sidman, C., Swett, R., Fik, T., Fann, S, and Sargent, B. 2006. A Recreational Boating Characterization of Sarasota County. Florida Sea Grant Publication TP-152. University of Florida, Gainesville, Florida.

West, N. 1982. Recreational Boating and Energy-Related Shipping on Naragansett Bay: A Study of Environmental Attitude and Behaviour. Report submitted to the Rhode Island CEIP, Governor's Energy Office. Providence, Rhode Island. 


\title{
Appendices
}

\section{Appendix A. Questionnaire and Correspondence}

\author{
Cover Letter
}

Sea fulfint Recreational Boating In Brevard County

\section{Dear Boat Owner / Operator,}

We are asking you to participate in a boating study being carried out in southwest Florida by the University of Florida Sea Grant Program. The study seeks to characterize boating in Brevard County waterways. Your responses will be very important to our efforts to help Brevard County prioritize and improve waterway access and maintenance, and to develop map-based boating products that enhance your recreational boating experience. There are no direct risks to you for participating in this study and we are enclosing a copy of "A Brevard County Boater's Guide" to thank you for completing and returning this questionnaire.

The questionnaire should take about 20 minutes to complete. We would appreciate it if you could complete and return it as soon as possible. We have provided a self-addressed, postage-paid return envelope. Please be assured that the information you provide will be held in the strictest confidence. Answers will NOT be traced to individuals and your name or address will NOT be made available to anyone else. Your participation is completely voluntary and you may withdraw your participation at any time without penalty. The questionnaire control number is used only to track survey returns so that we don't inconvenience you with reminder cards.

Only a small sample of Brevard County boaters have received this survey, so your input is very important. We recently completed a similar boating survey in the Charlotte Harbor area and it was a great success!

For questions about your rights as a research participant, you may contact the University of Florida Institutional Review Board at PO Box 112250, Gainesville, FL 32611 or 352-392-0433. If you have any questions about this survey or our products for boaters, you may contact Charles Sidman at the University of Florida (352) 392-6233, or by email at boatsurvey@ifas.ufl.edu

We are most grateful for your assistance in this important project. 


\section{Questionnaire Map}

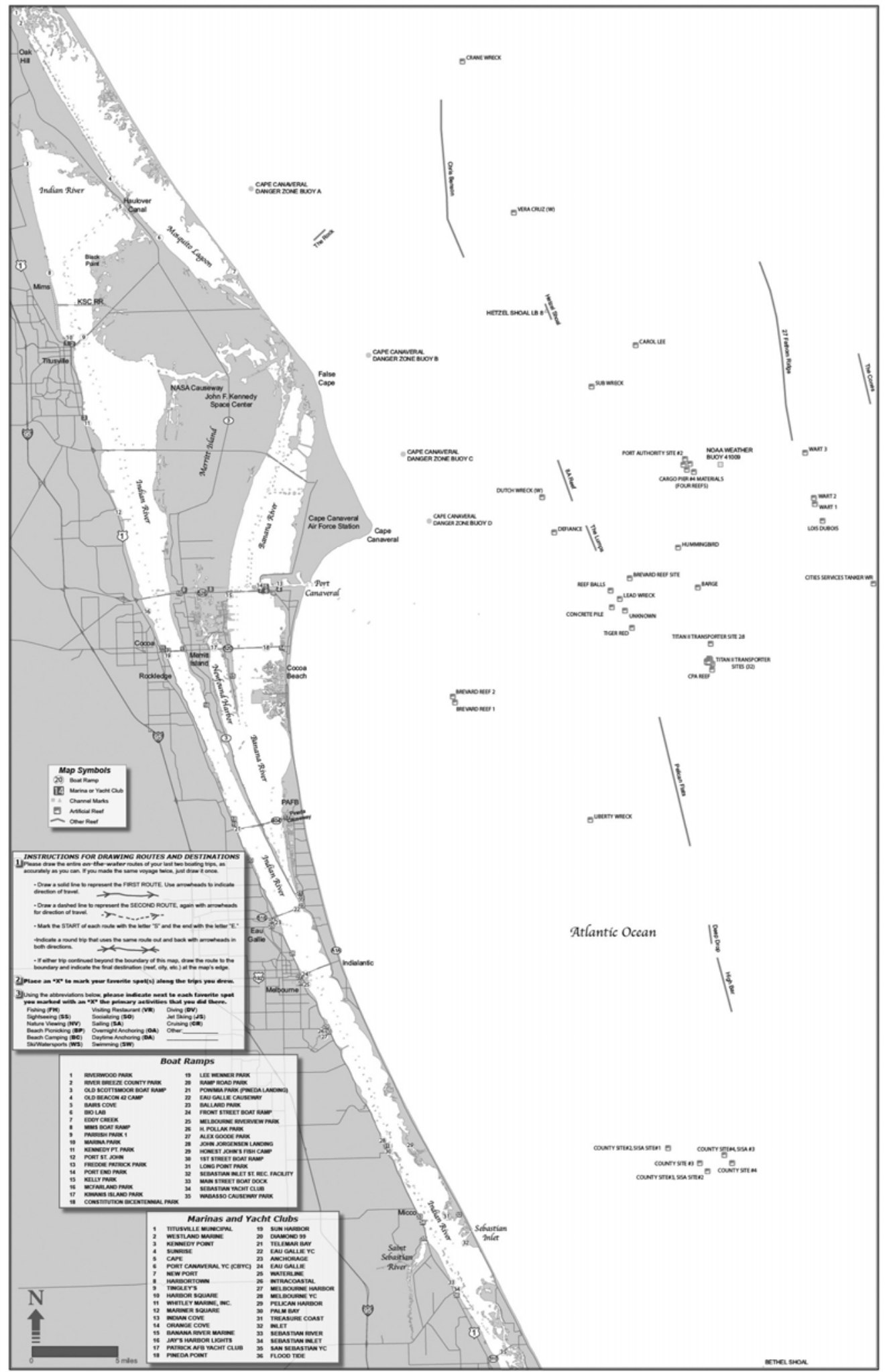




\section{Questionnaire}

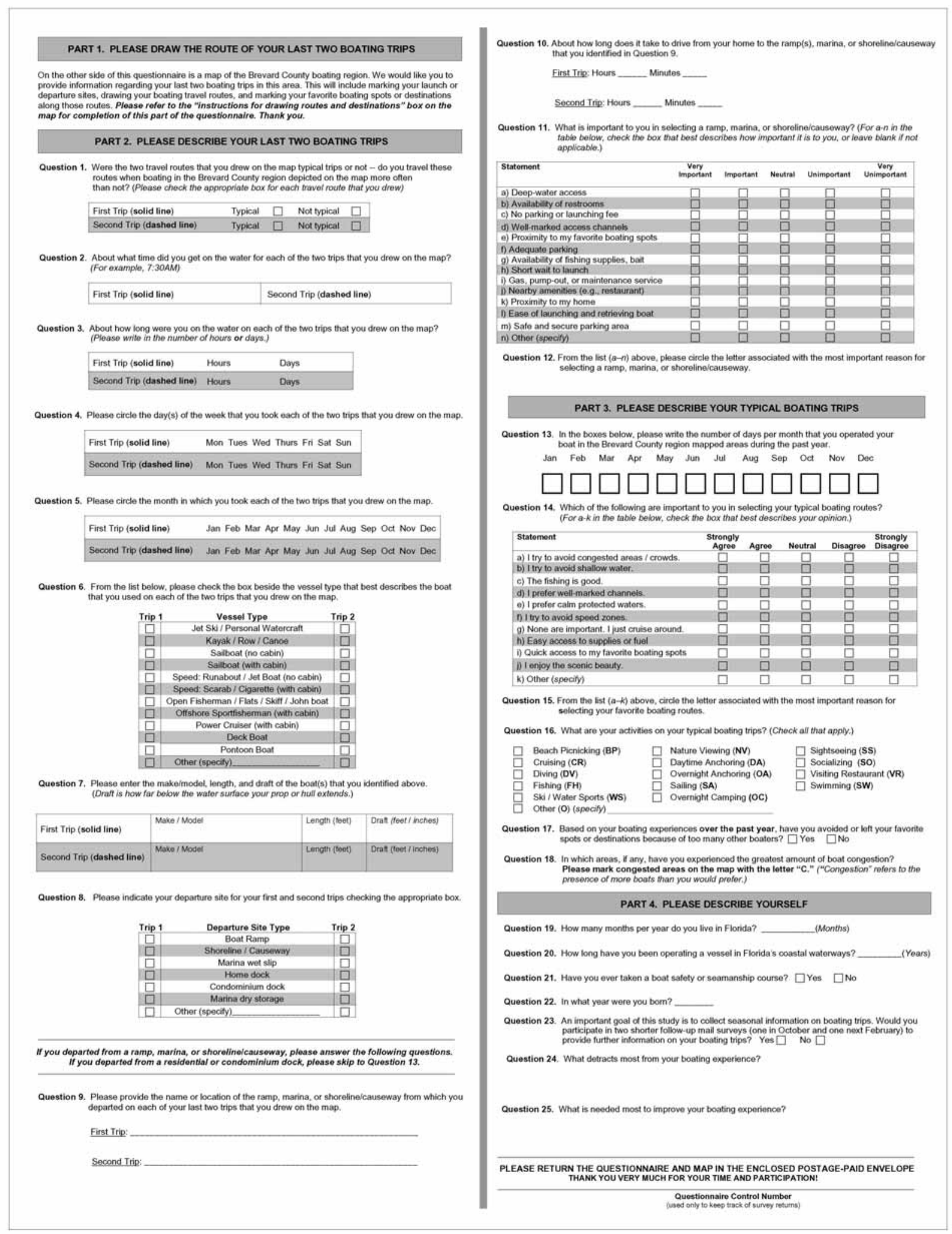




\section{Appendix B. Ramp Sampling Schedule}

Weekend and weekday ramp survey dates in chronological order.

\begin{tabular}{|c|c|c|c|c|c|c|}
\hline Month & Date & Day & & Month & Date & Day \\
\hline March & 11 & Saturday & & August & 24 & Thursday \\
\hline March & 12 & Sunday & & September & 2 & Saturday \\
\hline March & 18 & Saturday & & September & 3 & Sunday \\
\hline March & 19 & Sunday & & September & 8 & Friday \\
\hline April & 1 & Saturday & & September & 16 & Saturday \\
\hline April & 2 & Sunday & & September & 17 & Sunday \\
\hline April & 8 & Saturday & & October & 7 & Saturday \\
\hline April & 9 & Sunday & & October & 8 & Sunday \\
\hline April & 20 & Thurday & & October & 18 & Wednesday \\
\hline May & 5 & Friday & & October & 28 & Saturday \\
\hline May & 13 & Saturday & & October & 29 & Sunday \\
\hline May & 14 & Sunday & & November & 10 & Friday \\
\hline May & 15 & Monday & & November & 25 & Saturday \\
\hline May & 23 & Tuesday & & November & 26 & Sunday \\
\hline May & 27 & Saturday & & December & 4 & Monday \\
\hline May & 28 & Sunday & & December & 9 & Saturday \\
\hline June & 10 & Saturday & & December & 10 & Sunday \\
\hline June & 11 & Sunday & & December & 30 & Saturday \\
\hline June & 21 & Wednesday & & December & 31 & Sunday \\
\hline July & 1 & Saturday & & January & 8 & Monday \\
\hline July & 2 & Sunday & & January & 20 & Saturday \\
\hline July & 10 & Monday & & January & 21 & Sunday \\
\hline July & 22 & Saturday & & February & 6 & Tuesday \\
\hline July & 23 & Sunday & & February & 10 & Saturday \\
\hline August & 1 & Tuesday & & February & 11 & Sunday \\
\hline August & 12 & Saturday & & February & 24 & Saturday \\
\hline August & 13 & Sunday & & February & 25 & Sunday \\
\hline & & & & & \\
\hline
\end{tabular}

